\title{
Coded excitation and sub-band processing for blood velocity estmation in medical ultrasound
}

Gran, Fredrik; Udesen, Jesper; Jensen, Jørgen Arendt

Published in:

Proc. 153RD meeting of the Acoustical Society of America, The Journal of the Acoustical Society of America,

Publication date:

2007

Document Version

Publisher's PDF, also known as Version of record

Link back to DTU Orbit

Citation (APA):

Gran, F., Udesen, J., \& Jensen, J. A. (2007). Coded excitation and sub-band processing for blood velocity estmation in medical ultrasound. In Proc. 153RD meeting of the Acoustical Society of America, The Journal of the Acoustical Society of America, (Vol. 121, pp. 3154-3154). Acoustical Society of America.

\section{General rights}

Copyright and moral rights for the publications made accessible in the public portal are retained by the authors and/or other copyright owners and it is a condition of accessing publications that users recognise and abide by the legal requirements associated with these rights.

- Users may download and print one copy of any publication from the public portal for the purpose of private study or research.

- You may not further distribute the material or use it for any profit-making activity or commercial gain

- You may freely distribute the URL identifying the publication in the public portal 


\title{
Session 4aAA
}

\section{Architectural Acoustics: Troubleshooting in Room Acoustics}

\author{
Robin Glosemeyer Petrone, Chair \\ Jaffe Holden, 1453 3rd St. Promenade, Suite 335, Santa Monica, CA 90401
}

Chair's Introduction-8:45

Invited Papers

8:50

4aAA1. Acoustic enhancement in large indoor volumes. Steve Barbar (LARES Assoc., 30 Dunbarton Rd., Belmont, MA 02478)

Large indoor spaces present many unique challenges for controlling sound. If both speech and music must be simultaneously delivered with high fidelity in a large indoor volume, the challenges for sound delivery can become daunting. Carefully designed acoustical treatments and electro-acoustic systems can yield good speech intelligibility. These treatments, however, can be detrimental to the delivery of music. Integrating time-variant electronic acoustical enhancement allows both acoustical treatments and electroacoustic systems to be used more effectively. This presentation will discuss the electro-acoustic systems installed at the conference center for the Church of Jesus Christ of Latter Day Saints. Impulse response measurements indicate how portions of each system and architecture contribute to the listening experience at different locations in the venue.

9:10

4aAA2. The use of directional and binaural microphones for troubleshooting room acoustic problems. Peter Mapp (Peter Mapp \& Assoc., 101 London Rd, Copford, Colchester, Essex C06 1LG, UK, Petermapp@btinternet.com)

A common problem encountered when troubleshooting room acoustic issues (apart from the room exhibiting an overly long reverberation time or inadequate sound insulation) is the generation of late arriving reflections and echoes. The paper describes a number of techniques using both directional and binaural microphones as well as microphone array techniques for determining the direction, timing, and relative strength of such reflections. It is shown that in many situations, apparently highly directional microphones such as hypercardioid or rifle types, do not offer sufficient directional discrimination to be of much practical use. It is also shown that the inconsistent off axis frequency response of many such microphones can also lead to misleading analyses. Parabolic dish reflector microphones are shown to offer superior performance as do some microphone array techniques. It is shown that binaural measurements can also be a useful approach particularly with regard to assessing the subjective affect of reflections. The results of some early research into a novel extension to the Speech Transmission Index (STI) for assisting with acoustic trouble shooting will also be presented.

\section{9:30}

4aAA3. Interesting acoustics in the Salt Lake Tabernacle and the Latter Day Saints conference center. Sarah Rollins (Yantis Acoust. Design, 720 Olive Way, Ste. 1400, Seattle, WA 98101)

The Salt Lake Tabernacle and LDS Conference Center have interesting acoustical properties. Due to the elongated domed ceiling of the Tabernacle, there are audible focusing effects throughout the hall. In order to characterize the Tabernacle's unique acoustics prior to its seismic renovation, the acoustical properties of the hall were investigated through impulse response measurements and computer modeling. This paper will show how the measurements and model were used to see the focusing effects in the Tabernacle. The acoustics of the LDS Conference Center have also been extensively measured. This paper will show various methods that were used to investigate the sound focusing effects that were audible in the Conference Center.

9:50

4aAA4. Flutter echo at Pigott Auditorium. Basel Jurdy (Yantis Acoust. Design, 720 Olive Way, Seattle, WA 98101)

A flutter echo was easily apparent after completing the remodel of Pigott Auditorium at Seattle University in Seattle, Washington. Knowing that this was a possibility in the remodel design, walls were articulated in an attempt to avoid this phenomenon. The techniques used to investigate the problem, results of the investigation and the eventual remedy are presented. 
4aAA5. Programming remediation to a newly complete performing arts center. Carl J. Rosenberg, Jennifer Hinckley, and Jonah Sacks (Acentech Inc., 33 Moulton St., Cambridge, MA 02138, crosenberg@acentech.com)

A large new performing arts building at a university included an 800 -seat recital hall, proscenium theater, black box theater, large instrumental rehearsal room, choral rehearsal room, and practice rooms. It was designed with attention to acoustics concerns throughout, but the final results left the owner with a myriad of problems. These problems included poor hearing conditions in the large performance space, intrusion of exterior environmental noise, excessive mechanical system noise and vibration, and inexplicable lack of separation between rehearsal rooms that were separated by an STC 70+ wall. The authors parsed through the complaints and field conditions to extract a range of reasons for the problems. The paper presents the analysis techniques for these challenges and shows options that were developed for practical solutions.

\section{0:50}

4aAA6. Computer-aided long distance troubleshooting. Andrew N. Miller and Robert C. Coffeen (School of Architecture and Urban Design, Univ. of Kansas, 1465 Jayhawk Blvd., Lawrence, KS 66045)

A three-dimensional model of a religious worship facility was developed through the use of the EASE software package. Using the EASE Aura module, a binaural impulse response was created for three listening locations in the space. EASERA software was then used to analyze the impulse response data, and potential solutions to the problems observed were proposed. Each solution was then implemented in the model, and impulse responses were made for each scenario. Analysis after all proposed solutions were implemented in the model suggested that their real-world counterparts would prove successful. This paper raises the discussion of the effectiveness of long distance troubleshooting using modeling and analysis software.

\section{1:10}

4aAA7. Troubleshooting the Vivian Beaumont Theatre. J. Christopher Jaffe and Russell Cooper (Jaffe Holden Acoust., 114A Washington St., Norwalk, CT 06854)

Troubleshooting room acoustics can vary from the sublime to the ridiculous. This paper discusses some simple solutions to problems related to a lack of knowledge of basic acoustic characteristics. However, the main example in the paper relates to modifications made to the Vivian Beaumont Theatre at Lincoln Center over a period of many years. The Beaumont is a 1500-seat thrust theatre designed in the 1960s to house the Lincoln Center Repertory Company. Since opening night the theatre was plagued with poor speech intelligibility based on the facts that the seating capacity is too large for the thrust format and that the designers placed an immense proscenium stage house behind the thrust. The acoustic solutions implemented were based on discussions with two different managing directors, Joseph Pape and Bernard Gersten, and their different visions on how to mount productions in the space.

\section{Contributed Papers}

\section{1:30}

4aAA8. Lessons learned from room acoustics design and investigation (especially from using EASE room acoustics software) in our consulting practice. Joseph Bridger, Noral Stewart, and Aaron Farbo (Stewart Acoust. Consultants, 7406 L Chapel Hill Rd., Raleigh, NC 27607, joe@sacnc.com)

Experiences troubleshooting during the design of new buildings and investigation of existing acoustical problems in existing buildings, including churches, dining spaces auditoria, public meeting spaces, offices, and rehearsal spaces. Lessons learned and experiences with tools, such as EASE room acoustics software. Theoretical concepts to consider in designing box shaped rooms, cafeterias, rehearsal spaces, and churches. Tools and methods to consider in acoustical investigations.
4aAA9. Case studies of troubleshooting room acoustics problems using critical listening, field measurements, and computer models. Gary W. Siebein (Univ. of Florida, P.O. Box 115702, Gainesville, FL 32611-5702), Robert M. Lilkendey, Hyun Paek, and Edwin S. Skorski (Siebein Assoc., Inc., Gainesville, FL 32607)

Combinations of critical listening, field measurements of impulse responses and their associated metrics, and computer model studies were used in four projects to troubleshoot acoustical problems perceived by users and to study potential design solutions. Case study 1 is a 56-person firing range at a law enforcement training center nicknamed the Thunder Dome because of the excessive build-up of reflected and focused sound in the room. Case study 2 involved the diagnosis and correction of excessive loudness in a band practice room where instructors were threatening legal actions for hearing compensation claims against the school board. Case study 3 is a large multi-purpose performing arts center that suffered from many architectural acoustics and sound system design defects. Case study 4 involved the diagnosis and correction of sound focusing in an exclusive restaurant at a major resort that resulted from very subtle wall and ceiling shapes. The case studies illustrate the range of issues that can be studied with computer models and field measurements, as well as understanding the limitations of the techniques at the present time. 


\title{
Session 4aBB
}

\section{Biomedical Ultrasound/Bioresponse to Vibration: Coded Excitation}

\author{
Jonathan Mamou, Cochair \\ Riverside Research Inst., F. L. Lizzi Center for Biomedical Engineering, 156 William St., New York, NY 10023 \\ Michael L. Oelze, Cochair \\ Univ. of Illinois, Electrical and Computer Engineering, Beckman Inst., 405 N. Mathews, Urbana, IL 61801
}

\section{Invited Papers}

8:30

4aBB1. An acoustic spread spectrum technique for the study of outdoor noise propagation. David C. Waddington and Jamie A. Angus (Acoust. Res. Ctr., Univ. of Salford, Salford, M5 4WT, UK, d.c.waddington@ salford.ac.uk)

An investigation of the properties of a directly carrier-modulated code sequence modulation signal is presented for the study of outdoor propagation. The audible frequency carrier is biphase-modulated using a pseudorandom code sequence specially designed for simultaneous fine-time resolution and large-range ambiguity. An experiment is described in which the correlation properties of the spread spectrum signal are demonstrated, and the amplitude of the transfer functions between the receivers, together with accurate times-of-flight, are calculated from the cross-correlation of the measured acoustic spread spectrum signals. The results illustrate that an acoustic spread spectrum technique can provide significantly improved ways of measuring sound propagation outdoors. In particular, the fine-time resolution for time-of-flight suggests an application in acoustic tomography, for example in the investigation of nocturnal boundary layers, horizontal gradients, and of turbulence structures in the atmospheric surface layer. [Work supported by EPSRC UK.]

\section{$8: 50$}

4aBB2. Complex-valued coded excitations for acoustical channel measurements. Ning Xiang and Shu Li (Grad. Program in Architectural Acoust., School of Architecture, Rensselaer Polytechnic Inst., Troy, NY 12180)

Coded signals have been increasingly applied in acoustical/bio-ultrasonic measurements in order to increase the measurement quality or to cope with demanding requirements [Xiang and Schroeder (2003)]. Increasing interest in coded signals as acoustical excitations has prompted development of efficient correlation algorithms [Daigle and Xiang, J. Acoust. Soc. Am. (2006)]. These algorithms allow efficient processing of complex-valued sequences as needed in critical acoustical measurements, such as using simultaneous sources. Exploiting excellent correlation properties of some coded signals, this work applies complex-valued quadratic residue $(\mathrm{QR})$ sequences and Zech-logarithm sequences in both base-band acoustical channel measurements using sequential excitations and band-limited acoustical channel measurements using amplitude-modulation schemes. Numerical simulations and laboratory experiments carried out in this work demonstrate feasibility of the techniques. This paper discusses advantages and challenges when using coded excitations in potential acoustical applications.

\section{9:10}

4aBB3. Enhanced spatial resolution of dual-mode ultrasound arrays using coded excitation. Yayun Wan and Emad Ebbini (Dept. of Elec. and Comput. Eng., Univ. of Minnesota, Minneapolis, MN 55455)

A prototype 64-element $1-\mathrm{MHz}$ concave $(\mathrm{ROC}=100 \mathrm{~mm}$ ) DMUA has been shown to produce conventional synthetic aperture (SA) images in a field of view extending by $6 \mathrm{~cm}$ axially and $4 \mathrm{~cm}$ laterally (around its geometric center). With an $f_{\#}=0.8$ and a fractional bandwidth $\approx 40 \%$, the lateral and axial resolutions of this prototype were $1.1 \mathrm{~mm}$ and $2.6 \mathrm{~mm}$, respectively. We have investigated the use of coded excitation and pseudoinverse filtering for enhancing the axial resolution of the DMUA. SA images were formed from a $12 \mu \mathrm{s}$ linear chirp transmit pulse covering the frequency band of $0.5-1.5 \mathrm{MHz}$. The beamformed data was compressed using matched and regularized pseudoinverse filtering with a colored noise model. Image data were collected from a 3 D quality assurance phantom with an egg-shaped contrast target using the DMUA. The contrast ratio (CR) was 3.35, 7.8, and 6.6 for conventional pulse-echo, matched filter compression and inverse filter compression, respectively. In addition, axial and lateral speckle cell size parameters were calculated for the three methods. The axial resolution was $2.6,2.5$, and 1.65 for conventional pulse-echo, matched filter and pseudoinverse filter, respectively. Thus one can see that approximately $33 \%$ improvement in resolution was achieved without significant loss in CR or change in lateral resolution.

\section{9:30}

4aBB4. High resolution color flow-application of coded excitation in color flow imaging. Xiaohui Hao (18548 NE 57th St., Redmond, WA 98052, xiaohui.hao@ultrasonix.com)

Color flow imaging has suffered from the trade off between resolution and sensitivity ever since it was introduced. The requirement of blood flow sensitivity results in application of narrow bandwidth, long time duration pulses. The problem associated with this type of pulse is the severely degraded spatial resolution, which causes wall overwriting, blobby flow aesthetics, loss of detail flow 
hemodynamics, etc. Coded Excitation has been applied in B mode imaging to solve the similar tradeoff between SNR and resolution, but has not been so successful in color flow imaging. This is due to the fact that current color flow pulses are already at the regulatory limit and the focus of delivering more power for more sensitivity with coded excitation in color flow imaging. High resolution color flow imaging is an effort to introduce Chirp Coded-Excitation to achieve B-mode-like spatial resolution in color flow without sacrificing sensitivity. Wide bandwidth pulse with time bandwidth product greater than 1 is employed to bring in sensitivity/ penetration and resolution. As a result, better spatial definitions, less wall overwriting, and detailed flow hemodynamics are achieved with improved flow sensitivity. Corresponding practical application issues are discussed in this paper.

\section{9:50-10:10 Break}

\section{0:10}

4aBB5. Coded excitation and subband processing for blood velocity estmation in medical ultrasound. Fredrik Gran, Jorgen Arendt Jensen (Tech. Univ. of Denmark, Ctr. for Fast Ultrasound Imaging, Oersted-DTU, Bldg. 349, DK-2800 kgs. Lyngby, Denmark, fg@oersted.dtu.dk), Jesper Udesen, and Michael Bachmann Nielsen (Univ. Hospital of Copenhagen, Blegdamsvej 9, DK-2100 Copenhagen, Denmark)

This paper investigates the use of broadband coded excitation and subband processing for blood velocity estimation in medical ultrasound. In conventional blood velocity estimation a long (narrow-band) pulse is emitted and the blood velocity is estimated using an auto-correlation based approach. However, the axial resolution of the narrow-band pulse is too poor for brightness-mode (B-mode) imaging. Therefore, a separate transmission sequence is used for updating the B-mode image, which lowers the overall frame-rate of the system. By using broad-band excitation signals, the backscattered received signal can be divided into a number of narrow frequency bands. The blood velocity can be estimated in each of the bands and the velocity estimates can be averaged to form an improved estimate. Furthermore, since the excitation signal is broadband, no secondary B-mode sequence is required, and the frame rate can be increased. To increase the SNR for the broad-band excitation waveforms, coding is proposed. Three different coding methods are investigated: nonlinear frequency modulation, complementary (Golay) codes, and Barker codes. Code design is described for the three different methods as well as different ways of pulse compression for restoring axial resolution. The different methods were studied using an experimental ultrasound scanner and a circulating flow rig.

\section{Contributed Papers}

\section{0:30}

4aBB6. Pulse-compression ultrasound imaging using a 40-MHz annular array. Jonathan Mamou and Jeffrey A. Ketterling (F. L. Lizzi Ctr. For Biomed. Eng., Riverside Res. Inst., 156 William St., New York, NY)

High-frequency ultrasound allows fine-resolution imaging at the expense of limited depth-of-field and acoustic penetration depth. Codedexcitation imaging allows a significant increase in the signal-to-noise ratio (SNR) and acoustic penetration depth. A 40-MHz, five-element annular array with a focal length of $12 \mathrm{~mm}$ and a total aperture of $6 \mathrm{~mm}$ was made using a $9-\mu \mathrm{m}$ thick PVDF-TrFe membrane. The transducer was excited with an optimized, custom design, 4- $\mu$ s, linear chirp spanning 15 to 65 $\mathrm{MHz}$ that was deduced from ex vivo experiments and computer simulations. Images of a $12-\mu \mathrm{m}$ wire were generated to quantify lateral and axial resolutions. All 25 transmit/receive signal combinations were digitized and post-processed for compression and synthetic focusing. Compression consisted of linearly filtering the signals with the time-reversed excitation chirp modulated by a Dolph-Chebyshev window. Results showed that resolutions were not significantly degraded when compared to a conventional monocycle excitation and that SNR was improved by more than 14 dB. Images of mouse embryos showed marked improvements in imagequality. [Work supported in part by NIH Grant R21 EB006509.]

\section{0:45}

4aBB7. Improved scatterer size estimation from ultrasound backscatter using coded excitation and pulse compression. Michael L. Oelze and Steven Kanzler (Dept. of Elec. and Computer Eng., Univ. of Illinois at Urbana-Champaign, Urbana, IL 61801)

Scatterer size estimation from ultrasound backscatter has been used to improve the detection and classification of cancer. Estimates of scatterer size from tissues come from ultrasound backscattered signals that often have low signal-to-noise ratio (SNR). Coded excitation was explored as a means to increase the SNR of signals used in scatterer size estimation. The normalized backscatter coefficient was measured from tissue mimicking phantoms containing glass beads. The diameters of the glass beads were estimated by fitting the normalized backscatter coefficient to models for scattering from spheres [J. Acoust. Soc. Am. 23, 405-418 (1951)]. Two weakly-focused, single-element transducers ( $\mathrm{f} 0=5 \mathrm{MHz},-6 \mathrm{~dB}$ BW $=4.2 \mathrm{MHz}, \mathrm{f} / 3$, and $\mathrm{f} 0=10 \mathrm{MHz},-6 \mathrm{~dB} \mathrm{BW}=9.1 \mathrm{MHz}, \mathrm{f} / 4)$ were used in the measurements. For coded excitation, a linear FM chirp with a time bandwidth product of 40 was used. Three phantoms with average glass bead diameters of 30,45 , and $82 \mu \mathrm{m}$ were scanned. Preliminary results indicated that estimate bias was reduced versus penetration depth for the coded excitation compared to conventional pulsing. In addition, estimate variance using the coded excitation technique was reduced because the SNR was increased. [Work supported by start-up funds from the University of Illinois at Urbana-Champaign.]

\section{1:00}

4aBB8. An ultrasonic imaging speckle suppression technique by means of frequency compounding and coded excitation. Jose R. Sanchez and Michael L. Oelze (Dept. of Elec. and Computer Eng., Univ. of Illinois at Urbana-Champaign, 405 N. Mathews, Urbana, IL 61801)

A method for improving the contrast resolution of an ultrasonic $\mathrm{B}$-mode image is proposed by combining the speckle reduction technique of frequency compounding and the pulse compression technique of resolution enhancement compression (REC). Frequency compounding is known to suppress speckle in B-mode images to provide improvements in image quality and contrast resolution, but at the expense of deteriorating the axial resolution. The REC technique is used to enhance the bandwidth of the ultrasonic imaging system by using convolution equivalence. This enhanced bandwidth translates into improvements in axial resolution of the ultrasonic imaging system. Therefore, these improvements in axial resolution are exploited to improve contrast resolution. Improvements are quantified by using contrast-to-noise ratio (CNR) metric in simulations and experiments. In simulations, a phantom with a hyperechoic target was imaged by utilizing a single element transducer with a center frequency of 2.25 MHz. In experimental measurements, a single-element transducer $(f / 3)$ with a center frequency of $2.25 \mathrm{MHz}$ was used to image \pm 3 - and \pm 6 -dB cysts in an ATS 539 multipurpose tissue-mimicking phantom. Preliminary simulation and experimental results indicated that the CNR could be improved by up to $25 \%$ and $60 \%$, respectively, without sacrificing axial resolution compared to conventional pulsing methods. 
4aBB9. Spread spectrum sequence design for the study of outdoor noise propagation. Jamie A. Angus and David C. Waddington (Acoust. Res. Ctr., Univ. of Salford, Salford, M5 4WT, UK)

The design of carrier-modulated code sequence signals that can be used for the study of outdoor propagation, are presented. In particular, the construction of these signals such that they are robust to the time-variance of the outdoor acoustic path is discussed. The design of these signals so random code sequences in a way that is specially designed for simultaneous fine time resolution and large range ambiguity. A particular advantage of this signal is that an estimate of the time-varying Doppler can be derived by recovering the suppressed carrier from the received signal, and various means of extracting the Doppler are explained. Extensions of these signals to allow multiple simultaneous measurements are also described. [Work supported by EPSRC UK]

THURSDAY MORNING, 7 JUNE 2007

SALON A, 9:00 TO 10:35 A.M.

\title{
Session 4aEA
}

\section{Engineering Acoustics: Acoustical Measurements and Methods I}

\author{
Harold C. Robinson, Chair \\ Naval Undersea Warfare Center, Code 2131, 1176 Howell St., Newport, RI 02841-1708
}

Chair's Introduction-9:00

\section{Contributed Papers}

\section{9:05}

4aEA1. Remote acoustical reconstruction of cave and pipe geometries. Todd Schultz, David Bowen, Gladys Unger, and Richard H. Lyon (Acentech Inc., 33 Moutlon St., Cambridge, MA 02138)

Military and commercial interests desire to determine the geometry of caves, tunnels, and piping systems remotely. Advance knowledge of the geometry would allow military personnel to focus on the mission objectives rather than on cave exploration and mapping. Commercial applications of remote geometry mapping include determining locations of collapsed mines and tunnels for search and rescue missions, and determining locations of breaches or constrictions in pipelines. This research effort begins to extend acoustic pulse reflectometry to these applications and only requires simple measurements made at the waveguide entrance. This implementation first measures the acoustic reflection coefficient using a modified form of the two-microphone method and computes the waveguide impulse response. Then the system uses an inverse-scattering algorithm to solve for the cross-sectional area as a function of distance. Results are shown for a simple area expansion attached to a laboratory impedance tube with a diameter of $35 \mathrm{~mm}$, and for a pedestrian tunnel with a nominal square cross-section of $5.9 \mathrm{~m}^{2}$. The impedance tube estimates match the known cross-sectional areas to within $10 \%$. The results from the pedestrian tunnel show greater uncertainty due to frequency range limitations on the measurements, but can still capture important features of the geometry.

\section{9:20}

4aEA2. Acoustic and elastic radiative transfer theory in seismology. Jens Przybilla, Michael Korn (Inst. of Geophys. and Geology, Leipzig Univ., 04103 Leipzig, Talstr. 35, Germany, jprzybill@web.de), and Ulrich Wegler (Seismologisches Zentralobservatorium Graefenberg, D-91052 Erlangen, Mozartstr. 57, Germany)

Inversion results that were obtained by applying the acoustic and elastic radiative transfer theory to seismological data will presented. Scattering of seismic wave fields within the earth is documented by the pulse broadening of direct wave trains and the appearance of coda waves following the direct waves. The pulse broadening is caused by forward-scattered waves and the coda by wide-angle scattering. Random media are used as models for the heterogeneities of the earth. The energy transfer equations are solved with Monte Carlo methods. The results obtained with the acoustic and elastic theory for the transport scattering coefficients and absorption coefficients are compared with each other. It shows that scat- tering is the main process for lower frequencies. For higher frequencies intrinsic absorption is dominant. The results for elastic and acoustic radiative transfer are the same if strong forward scattering is assumed. For lower values of anisotropy the results are different.

\section{9:35}

4aEA3. An investigation into the relationship between sound intensity and normal surface velocity. Mark Boyle (School of Mech. and Aerosp. Eng., Queen's Univ. of Belfast, Ashby Bldg., Stranmillis Rd., Belfast, BT9 5AG, mboyle31@qub.ac.uk), Matthew Cassidy, Richard Cooper, Richard Gault, and Jian Wang (Queen's Univ. of Belfast, Stranmillis Rd., Belfast, BT9 5AG, UK)

The analysis of sound characteristics using boundary element methods (BEM) requires accurate sound source data of normal surface velocity or pressure as boundary conditions. However, direct measurements of normal surface velocity are not easily acquired while sound intensity measurements are relatively easy to obtain. In this study the relationship between near field sound intensity and normal surface velocity is investigated. The study first considers correlated and uncorrelated sound sources in the frequency range of 63 to $1000 \mathrm{~Hz}$. A matrix of sound intensity measurements on well-distributed points in the near field of the sound source is collected. A model of the relationship between sound intensity and volume velocity is developed and used to compute the normal surface velocity of the sound source from sound intensity measurements. The normal surface velocities are then used as the boundary conditions for a BEM model of the sound source that predicts the sound intensity at the original measurement points. The measured and predicted values are compared in order to validate the model. The differences of predicted and measured sound intensities that are within $\pm 3 \mathrm{~dB}$ are considered sufficiently accurate.

\section{9:50}

4aEA4. An integrated optical microphone test-bed for acoustic measurements. Caesar T. Garcia, Neal A. Hall, F. Levent Degertekin, Baris Bicen, Kamran Jeelani, and M. Shakeel Qureshi (Dept. of Mech. Eng., Georgia Inst. of Technol., 771 Ferst Dr. NW, Atlanta, GA 30332, levent.degertekin@me.gatech.edu)

A diffraction-based optical detection method for microphone applications has been demonstrated previously [Hall et al., J. Acoust. Soc. Am. 118, 3000-3009 (2005)]. This method, coupled with proper integration 
techniques can produce precision measurement microphones with $24 \mathrm{dBA}$ noise levels and suitable bandwidths. Thus far, these characterization studies have been performed using experimental setups, which would disturb the acoustic field due to size and non-symmetric features. In these regards, previous optical microphone test beds have been inadequate experimental platforms. This has motivated the development of a more robust integrated instrumentation microphone package for future testing and characterization. In order to meet the size restrictions for such an optical microphone platform, vertical cavity surface emitting lasers are used as light sources and small photodiode arrays are used to detect intensity variations in refracted orders of the optical detection method. The overall dimensions and shape of the package are comparable to commercially available half-inch calibration microphones and impose minimal sound field disturbance. The design is adapted to allow simple replacement and remounting for multiple microphone testing including biomimetic directional microphones [Miles et al., J. Acoust. Soc. Am. 98, 3059-3070 (1995)]. [Work partially supported by NIH Grant 5R01DC005762-03 and the Catalyst Foundation.]

\section{0:05}

4aEA5. Design and implementation of a three-dimensional seven microphone acoustic intensity probe for measuring sound properties from various sources. Khalid Miah and Elmer Hixson (Elec. and Computer Eng. Dept., Univ. of Texas, Texas 78712, miah@mail.utexas.edu)

Based on existing theoretical models of multi-dimensional crossspectral methods for calculating sound pressure and particle velocities, this report has derived and/or extended models of intensity, energy densities, and specific acoustic impedance for seven microphone configurations. Array configuration, spacing, and overall implementation procedure is also outlined in this report. Experimental data has confirmed the validity of the analytical solution for different sound sources in both anechoic and rever- beration room environment. Sound sources of different frequency bands [broadband $(100 \mathrm{~Hz}-7 \mathrm{KHz})$, low $(100 \mathrm{~Hz}-1 \mathrm{KHz})$, and high $(1-7 \mathrm{KHz})$ ] was used separately to compare accuracy and performance of the acoustic probe. Signal processing techniques and software implementation with potential errors and limitations of the system are explained. These would provide a framework for further refinement of instrumentation aspects of the acoustic probe. Potential applications and users of the probe are also described in this report.

10:20

4aEA6. Phonometrical precision measurements during a high end classical music live recording and four standardized recording systems' signal comparison. Franco P. A. Policardi (DIENCA, Universit di Bologna, Viale Risorgimento 2, 40136 Bologna, Italy, franz.policardi@mail.ing.unibo.it)

Discographical sound recording process is very seldom accompanied by real sound pressure, intensity and level measurements. Usually available data are often referred to mathematical extrapolations calculated on sound recording audio signals. This article investigates the situation defined as "real" from a scientifical point of view, having as a referring base a Swedish precision sound level meter fresh from calibration, during the stereophonic multichannel live recording session of the Philharmonische Camerata Berlin. Comparison among reference signal and eight high quality microphone capsules signals coming from four different stereophonic standardized microphone systems devoted to discographical purpose recording (XY, ORTF, Binaurale and AB) shows big differences among the respective signals and the same recording spot measurement system position. It is noticeable how differences even higher than $6 \mathrm{~dB}$ can make microphone positioning particularly critical. A standardizing method is proposed.

\title{
Session 4aED
}

\section{Education in Acoustics: Tools for Teaching Acoustics}

\author{
Edward J. Tucholski, Chair \\ U.S. Naval Academy, Physics Dept., 572C Holloway Rd., Annapolis, MD 21401
}

Chair's Introduction-10:00

\section{Contributed Papers}

\section{0:05}

4aED1. An investigation of Rubens flame tube resonances. Michael D. Gardner (N283 ESC Brigham Young Univ., Provo, UT 84602), Gordon Dix (JBL Professional, Northridge, CA 91329), Kent L. Gee, and Jarom Giraud (Brigham Young Univ., Provo, UT 84602)

The Rubens flame tube is a $100+$ year-old teaching demonstration that allows observers to visualize acoustic standing wave behavior [H. Rubens and O. Krigar-Menzel, Ann. Phys. (Leipzig) 17, 149 (1905)]. Flammable gas inside the tube flows through holes drilled along the top and flames are then lit above. The tube is closed at one end and is driven with a loudspeaker at the other end. When the tube is driven at one of its resonance frequencies, the flames form a visual standing wave pattern as they vary in height according to the pressure amplitude in the tube. Although the basic performance of the tube has been explained [G. Ficken and C. Stephenson, Phys. Teach., 17, 306-310 (1979)], a previously unreported characteristic of some flame tubes has been observed and studied. This phenomenon involves an upward shift of the natural frequencies of the lower modes from what would ordinarily be expected in a closed-closed tube. Results from a numerical model suggest that the shift is primarily due to a Helmholtz resonance effect created by the drilled holes. The numerical results are compared to experimental findings and discussed.

10:20

4aED2. Collocated proportional-integral-derivative (PID) control of acoustic musical instruments. Edgar Berdahl and Julius Smith III (Ctr. for Comput. Res. in Music and Acoust. (CCRMA), The Knoll, 660 Lomita, Stanford, CA 94305, eberdahl@ccrma.stanford.edu)

The dynamic behavior of an acoustic musical instrument can be drastically modified by closing a feedback loop around a single sensor and actuator. Proportional-Integral-Derivative (PID) control provides for a simple yet effective paradigm, whose analysis is simplest for collocated systems. The analysis is further simplified when the musical instrument is modeled by a single, lightly damped mass-spring-damper system. This example is presented as a pedagogical laboratory exercise (http:// ccrma.stanford.edu/realsimple/pidcontrol) within the framework of the Re- 
alSimPLE project (http://ccrma.stanford.edu/realsimple). Students learn what physical models are, why they are useful, what the basic ideas behind feedback control are, and how they may be applied to a plucked string. A plucked string model is implemented with a digital waveguide in the Pure Data environment. Given the waveguide model and the theoretical results from the simplified mass-spring-damper model, students are asked to find PID coefficients that result in particular controlled instrument behaviors (increase in damping, decrease in pitch, etc.). At the close of the talk, a few more advanced topics are covered. In particular, a proof is presented explaining why damping with collocated velocity feedback is stable no matter how large the feedback loop gain is. [Work supported by the Wallenberg Global Learning Network.]

\section{0:35}

4aED3. Effectiveness of Physlet computer animations for enhancing student learning of acoustic principles in a course for non-science majors. Andrew A. Piacsek and Ian Wright (Dept. of Phys., Central Washington Univ., Ellensburg, WA 98926-7422)

A set of interactive computer animations of acoustic phenomena was created using the Java-based PHYSics appLETS (or physlets) developed at Davidson College [Christian and Belloni, 2001]. These animations allow the user to adjust certain parameters of a modeled system and view - in real time - the corresponding change in the physical behavior of the system. Because the animations are accessed via the web and can run on any computer (using Java), students can work with them in many different settings. The goal is to help students with limited math skills who enroll in PHYS103 "Physics of Musical Sound" at Central Washington University develop an intuitive understanding of basic acoustic phenomena and to assist them in applying quantitative reasoning to predict the behavior of musical systems. These newly-created acoustics and vibration physlets will be used for the first time during the Spring term, 2007, in the PHYS103 course taught by the author. Students will be given a quiz before and after working with the physlets on various topics to assess the effectiveness of this pedagogical tool.

\section{0:50}

4aED4. Using technology to enable people of all abilities to explore music. Alastair C. Disley, David M. Howard, and Andrew D. Hunt (Dept. of Electron., Univ. of York, Heslington, York, YO10 5DD, UK)

MIDI Creator is a commercially available programable device that converts sensor inputs to MIDI data, which can then directly trigger sound or be used as controls for more complex environments. A variety of sensors are available, including pressure-sensitive floor pads and ultrasonic beam controllers. The authors present two applications of this technology, which illustrate the system's ability to be used in music education and music therapy environments. In the first instance, two rows of floor pads were used as controls for a game projected onto the floor. The default game was a co-operative ping-pong variant, in which users were encouraged to work together to achieve the highest number of volleys. Other options included musical games, where users could co-operate to produce harmonies. All ages and abilities, including wheelchair users, were able to take part on an equal basis. In the second instance, a simple potentiometer was explored as an input device. School children rotating the potentiometer caused the MIDI Creator to play a scale and light lamps corresponding to the notes being played. The children then decided which notes lit which lamps. Other uses of MIDI Creator in the areas of music education and music therapy are summarized.

\section{1:05}

4aED5. Physics of a balloon lens. Derek C. Thomas, Kent L. Gee, and R. Steven Turley (Dept. of Phys. and Astron., Brigham Young Univ., N283 ESC, Provo, UT 84602, derekcthomas@ gmail.com)

A balloon filled with a gas that has a different sound speed than that of air has been used by science teachers in the past as an acoustic lens. One purpose of the lens is to show refraction of sound waves as an analogy to geometric optics [H. Kruglak and C. C. Kruse, Am. J. Phys. 8, 260-261 (1940)]. This paper discusses the physics involved with the balloon lens demonstration. In order to determine the validity of a balloon as a classroom demonstration of an acoustic lens and to understand the corresponding phenomena, the problem has been considered analytically, numerically, and experimentally. The results show that although a geometric analogy is a valid first order approximation, scattering theory is often required to fully understand the observed phenomena. Thus, this demonstration can be adapted to a wide range of students, from those learning basic principles of refraction to mathematically advanced students studying scattering.

\section{1:20}

4aED6. Combining physical REALity with SIMulations in pedagogical laboratory experiments. Edgar Berdahl, Nelson Lee, Ryan Cassidy, and Julius Smith III (Ctr. for Comput. Res. in Music and Acoust. (CCRMA), The Knoll, 660 Lomita, Stanford, CA, 94305, eberdahl@ccrma.stanford.edu)

The RealSimPLE project provides teachers with a modular collection of online musical acoustics laboratory experiments, including experiments on elementary wave motion, vibrating strings, pipes, sensors, digital waveguides, psychoacoustics, PID control, sound cards, and more. Hands-on laboratory sessions are complemented by pedagogical computer-based simulations or animations of the same systems. This dichotomous approach illustrates both the behavior of the real system together with corresponding idealized theoretical simulation. Moreover, the simulations can illustrate hard-to-measure details of the real system, or highlight details which easily escape the eye or ear. In this way, the traditional lab bench is enhanced rather than replaced. Detailed instructions help teachers and/or students easily construct the minimal amount of lowcost laboratory equipment needed. The RealSimPLE website (http:// ccrma.stanford.edu/realsimple) also includes rollover "pop-up" definitions for many technical terms. These definitions (within HTML pop-up overlays) include links to more-detailed definitions and examples, allowing website visitors to easily dig deeper down through the tree of prerequisite terms, thereby filling in any knowledge-gaps they may have. A motivated student anywhere in the world with a basic math and physics background can obtain advanced graduate-level knowledge from the website in a selfpaced, demand-driven manner. [This work was supported by the Wallenberg Global Learning Network.] 


\title{
Session 4aMU
}

\section{Musical Acoustics and Physical Acoustics: Flow Dynamics in Musical Instruments}

\author{
Rolf Bader, Chair \\ Univ. of Hamburg, Inst. of Musicology, Neue Rabenstr. 13, 20354 Hamburg, Germany
}

Invited Papers

9:00

4aMU1. Turbulence flow modeling using combined compressable Euler and molecular dynamic simulation (MDS) methods. Rolf Bader (Insitute of Musicology, Univ. of Hamburg, 20354 Hamburg, Germany)

Typical flows found with musical instruments as well as turbulent behavior are modeled. Here the problem of using many elements to model the system precisely resulting in very long simulation time is avoided using molecular dynamic simulation techniques, where the flow is represented by moving particles that can be at any position in space and so act as an alternative to small grid lengths. On the other hand, the coupling between the flow and the acoustic wave caused by oscillations of that flow is modeled using the compressable Euler model. Here, the equation of continuation is used for modeling a time dependency of the pressure and is thus capable of combining the models. Both models are presented on their own and also in several possible combinations. The flow and pressure distribution behavior of possible couplings between these methods is then discussed in terms of comparison with experimental data, modeling stability, and modeling speed.

\section{9:25}

4aMU2. Turbulence: Geometrical and statistical aspects. R. Friedrich (Inst. of Theoretical Phys. Westfälische Wilhelms-Universität Münster, Wilhelm-Klemm-Str. 9, 48149 Münster, Germany)

Turbulent flows are characterized by the transport of energy across scales. This energy transport, which frequently is denoted as energy cascade, is related to the formation of hierarchically organized spatio-temporal structures. A statistical treatment of velocity increments of turbulent ows thereby shows that the statistics are strongly intermittent exhibiting large deviations from Gaussian statistics. In this talk we shall give an overview on recent results concerning the analysis of Eulerian as well as Lagrangian velocity statistics, which allows one to characterize, understand, and model the small scale statistics of turbulent flows by means of stochastic processes. Furthermore, we review attempts to relate the statistical properties of small scale turbulent flows to the geometry of vortical structures in the flow field.

9:50

4aMU3. Flutelike instruments: A perfect joint between acoustics and hydrodynamics. Benoit Fabre (Inst. Jean Le Rond d'Alembert, Univ. Pierre et Marie Curie, 11, rue de Lourmel, F-75015 Paris, France)

The oscillation in flutelike instruments results from the aeroacoustic coupling of an hydrodynamic mode of a jet with an acoustic mode of a resonator. Since musical instruments are empirically optimized oscillators, the modeling of flutes appears as a test bench for different approaches. Complementary descriptions have been developed in the literature from the point of view of acoustics or from the point of view of hydrodynamics. Unfortunately, the basic hypothesis in both fields are not easily compatible: for instance, as a first approximation, pipe acoustics can be studied neglecting the influence of mean flow, while the jet instability can be studied neglecting the influence of compressibility. In the presentation, we will develop an aero-acoustic approach to the oscillation in flutelike instruments, that focuses on the different approximations that need to be carried. We will emphasize on the limited range of control parameters that the current models may describe. The different elements will be discussed as well as the traditional custom to study the system as a lumped model.

10:15-10:30 Break

10:30

4aMU4. The acoustic consequences of time-dependent changes of the vocal tract shape. Brad Story (Univ. of Arizona, P.O. Box 210071, Dept. of Speech, Lang., and Hearing Sci., Tucson, AZ 85721)

During singing or speech production, the human vocal tract is a nearly continuously changing conduit through which sound propagates. As the vocal tract shape changes, its resonance frequencies also continuously vary, shaping an excitation signal into a sequence of vowels and consonants. This presentation will focus on modeling the time-dependent movements of the vocal tract shape and their acoustic consequences. Based on data collected with MRI and x-ray microbeam techniques, a kinematic model of the vocal tract area function has been developed that allows for efficient specification of time-dependent cross-sectional area changes in an acoustic waveguide. When coupled with a voice source, the result is a basic simulation of the sound production process from which pressures and airflows are generated. The components of the model will be presented and then used to demonstrate some timedependent relations between the vocal tract shape and resulting acoustic characteristics. [Work supported by NIH R01-DC04789.] 
4aMU5. Synchronization of organ pipes as a paradigm for nonlinear acoustical oscillations. Markus Abel and Steffen Bergweiler (Dept. of Phys., Universität Potsdam, Am Neuen Palais, Postfach 601553, D-14415 Potsdam, Germany)

From measurements on organ pipes, it has been known for a long time that the so-called Mitnahme Effekt can lead to a mutual influence of organ pipes by each other. The same holds for external driving of pipes by acoustical sources of well-defined frequencies. A locking of two differently tuned pipes to a single frequency, or to the frequency of the external driving, respectively, is observed. We measure the dependence on the coupling by varying the distance of the pipes. In great detail, we measure the dependence on the signal strength in the case of the external driving. As a result, we find a so-called Arnold tongue over a range of $50 \mathrm{~dB}$, i.e., for extremely weak driving, one still finds synchronization to a common frequency. We apply the very general theory of synchronization from the point of view of nonlinear vibrations and apply it to the experimental data from organ-pipe measurements. Surprisingly, it is possible to capture the main features of the phenomenon by nonlinear oscillator equations, coupled to each other. A full explanation includes a good understanding of the aeroacoustics producing the sound of an organ pipe.

\title{
Session 4aNSa
}

\section{Noise: Environmental Noise in National Parks}

\author{
Nancy S. Timmerman, Chair \\ 25 Upton St., Boston, MA 02118-1609
}

\section{Invited Papers}

\begin{abstract}
8:00
4aNSa1. Acoustic monitoring and resource management in national parks. Kurt Fristrup (Natural Sounds Program, Natl. Park Service, 1201 Oakridge Dr., Ste. 100, Fort Collins, CO 80525)

The Organic Act of 1917 and other legal mandates and authorities require the NPS to protect and restore park resources. Acoustic monitoring has been conducted in 43 park units. These studies have documented sound levels (in $\frac{1}{3}$ octave bands) and identified sources of noise. Analyses show that backcountry sites can be extraordinarily quiet, but noise events occur at all locations. It is clear that noise presents two threats to park resources: chronic degradation of listening opportunities as well as acute effects from prominent events. The NPS is developing metrics that assess the extent to which noise degrades the opportunities for visitors to experience the sounds intrinsic to the park unit. These metrics also apply to the degradation of aural awareness for wildlife, which can affect foraging success, predator avoidance, and acoustic communication.
\end{abstract}

\section{$8: 25$}

4aNSa2. Modeling noise and visitor use in national parks. Kenneth J. Plotkin (Wyle Labs., 241 18th St. S., Ste. 701, Arlington, VA 22202), Steven R. Lawson (Virginia Polytechnic Inst. and State Univ., Blacksburg, VA 24061), and Kurt M. Fristrup (Natl. Park Service, Fort Collins, CO 80525)

The natural soundscape in National Parks is degraded by the intrusion of human-made noises. One usually thinks of the intrusive sounds as those from mechanical devices: aircraft, snowmobiles, off-highway vehicles, tour buses, generators, etc. Noise Model Simulation (NMSim) developed for the Park Service was designed to address those sources. There is, however, often a significant nonmechanical human noise source: the humans themselves. Visitors traveling in groups tend to talk to each other, and that speech can be an intrusive noise source. Newman et al. conducted a listening exercise in Muir Woods National Monument in 2005 to assess the nature of these human sounds. In the fall of 2006 Lawson et al. conducted noise and trail use monitoring in the Great Smoky Mountains National Park, which quantified visitor-generated sound levels and provided input to trail use modeling. Those data provide inputs to NMSim, so that the exposure of visitors to visitor noise and the impact of visitor noise on the soundscape can be quantified. The Smokies study will lead to a general merger of noise and trail use modeling, so there will be tools for this aspect of soundscape modeling and preservation in National Parks.

4aNSa3. Grand Canyon: Restoration of natural quiet (current status). Dickson J. Hingson (Sierra Club, Natl. Parks and Monuments Committee, 275 S. River Run Rd. \#3, Flagstaff, AZ 86001, dhingson@infowest.com)

Mandated restoration efforts to reduce extensive aviation noise intrusions into the wild soundscape of Grand Canyon National Park will be reviewed, in context of underlying, protracted political controversy. This is timely, for the approaching, presidentially directed due date is 22 April 2008, under the National Parks Overflights Act of 1987, for the "substantial restoration" of "natural quiet," pursuant to noise standards/specifications of the National Park Service, with due consideration to the FAA for ensuring safety of aircraft operations. The draft EIS and "Preferred Alternative" (release due spring 2007) will therefore vigorously resurface scientific review of primary and supplemental noise indicators and standards appropriate to national parks. Grand Canyon, having large wilderness/backcountry areas and renown, is a particularly iconic focus for scrutiny. The restoration will be viewed against actualities 
of (1) the large number/types of aircraft operations at all altitudes above the park; (2) demanding management objectives and large size of Grand Canyon; and (3) its extremely quiet natural soundscape (which characterizes most desert and Colorado Plateau national parks.) Visitor experience values, including "wilderness character" and sound as resource are offered as ultimate concerns, often clashing with industrial tourism and its associated values of aerial perspective, speed/convenience, and economic return for tour operators.

4aNSa4. Protecting natural soundscapes with an adequate margin of safety. Richard Horonjeff (81 Liberty Sq. Rd., \#20-B, Boxborough, MA 01719) and Grant Anderson (Harris Miller Miller \& Hanson Inc., Burlington, MA 01803)

The natural ambient sound environment in park-like settings not only provides a masker for inappropriate sounds, but also affords different acoustic experiences to the park visitor due to its dynamic nature. At a single location, for example, the absence of wind and water movement may offer periodic opportunities to experience an environment completely devoid of audible sound. At the same location wind blowing gently through nearby conifers may offer a feeling of tranquility. In late spring the sound of water in a nearby stream may provide yet another experience. Each circumstance generates a different ambient sound environment, both for soundscape enjoyment as well as masking inappropriate sources (usually human activity). This dual role creates challenges in soundscape management and planning and makes difficult the use of a single ambient descriptor to protect a sites multiple non-concurrent soundscape resources. This paper examines soundscape impairment from a damage risk perspective by assuming independent, quasi-random arrivals of various soundscapes and human activity. Actual data from three western U.S. parks are used to establish reasonable parameter values for the analysis. The paper develops impairment risk with an adequate margin of safety in line with philosophies of the EPA Levels Document of 1974.

9:40

4aNSa5. Cleaning up 20th century acoustical trash from national parks: 10 ways to quiet our parks. Leslie D. Blomberg (Noise Pollution Clearinghouse, Box 1137, Montpelier, VT 05601-1137, npc@nonoise.org)

The history of noise in the 20th Century follows a consistent pattern, first the invention of new noise sources, then the growth in use of those sources, and finally the spread of those noises into previously quiet areas. Our National Parks have experienced that same pattern of invention, growth, and spread. Moreover, those previously quiet areas that succumbed to 20th Century noises often included our Parks and wildlands. Since most of the noises degrading National Parks are 20th Century inventions, this paper will examine the historical context of todays park noise, with an emphasis on developing policy options to quiet our National Parks.

\section{0:05-10:20 Break}

10:20

4aNSa6. A low-complexity environmental noise monitoring system for unattended operation in remote locations. Robert D. Collier (Thayer School of Eng., Dartmouth College, Hanover, NH 03755) and Kenneth Kaliski (Resource Systems Group, Inc., White River Junction, VT 05001)

There is a need to measure environmental noise in remote locations such as national parks over extended periods of time with a simple, readily deployed, robust, unattended data acquisition system. The original Dartmouth College prototype system was demonstrated in 2001 [Kaliski et al., Proceedings NOISE-CON 2001, October 2001]. The improved Dartmouth system is described in a patent awarded to Dartmouth College in August 2006. The system conforms to ANSI/IEC Type I and Type II standards. It samples, processes, and stores 1-s A- and C-weighted equivalent sound level (Leq) values over a period of about 2 weeks. In addition, maximum, minimum, and statistical percentile values are stored in nonvolatile memory. The instrument is powered by a rechargeable battery backed up by a solar power converter. A communication interface provides for remote control. Separate software is provided to display statistical information and detection and classification clues based on user-selected time period events. The capabilities of the improved Dartmouth system are demonstrated in a selected measurement program.

\section{0:45}

4aNSa7. Anthropogenic contribution to the acoustic environment of Mexican spotted owls in the Gila National Forest, New Mexico. Ann E. Bowles, Samuel L. Denes (Hubbs-SeaWorld Res. Inst., 2595 Ingraham St., San Diego, CA 92109, abowles@hswri.com), Chris Hobbs, Kenneth L. Plotkin (Wyle Labs., Arlington, VA 22202), and Elizabeth Pruitt (GeoMarine, Inc., Hampton, VA 23666)

We monitored the acoustic environment of Mexican Spotted Owls using an array of Larson-Davis (LD) sound level meters (SLMs) from 2000 to 2005. Thirty-nine LD-820 and LD-824 SLMs were deployed in a distributed array across a $20 \mathrm{~km} \times 24 \mathrm{~km}$ area, collecting two-second time interval data as continuously as possible mid-April to July. Although the study was designed to monitor low-flying military jets, SLMs collected over $350,000 \mathrm{hr}$ of usable environmental noise data. The data were summarized by time, average level, and on an energy basis. The most important identifiable sources differed depending on metric. On a time basis, the ambient was the greatest contributor, followed by biotic sources and thunder. Regional jets were unexpectedly important, accounting for $2 \%$ of the total, far more than military jets. However, military jets and thunder accounted for most of the exposure to high amplitude noise (proportion of 2-s samples with LAeq i $60 \mathrm{dBA}$ ). In terms of cumulative sound exposure, thunder was the greatest contributor, followed by ambient noise and military jets. The sources most likely to impact owls will depend on the critical features of noise from their perspective; identifying these features is the focus of future analysis [Work supported by U.S. Air Force ACC/ CEVP] 


\title{
Contributed Papers
}

11:10

4aNSa8. Characterizing natural soundscapes and understanding human response to human-caused noise in a Hong Kong country park. Kin-che Lam, Kwai-cheong Chau, and Lawal M. Marafa (Dept. of Geography \& Resource Management, Chinese Univ. of Hong Kong, Shatin, N.T., Hong Kong)

A study was undertaken in a country park of Hong Kong to characterize the natural soundscapes and determine the affective response of visitors to natural and human-caused sounds. Located on the eastern extremity of Hong Kong, the country park is easily accessible and characterized by sandy beaches, rocky shores, rugged slopes, and wooded ravines nested among silent hills and mountains. The country park is exposed to varying levels of transportation noise (motor vehicle, aircraft) and visitor-caused noise. The soundscapes of a variety of natural landscapes were characterized by sound walks and acoustical and psycho-acoustical analysis of 15min and 24-h sound recordings. Interviews with the country park visitors revealed that they preferred soundscapes with natural and joyful attributes. A questionnaire survey was undertaken in several landscape-soundscape settings with different exposure levels of human-caused noise. The results show that while the visitors were slightly bothered by extraneous noise, the degree of annoyance was significantly determined by the natural set- ting, the background soundscape, and the type of activities engaged in. The findings provide an empirical basis to formulate management strategies of natural soundscapes and contribute to a growing body of research on the potential impacts of human-caused sound in country parks.

\section{1:25}

4aNSa9. Review, comparison, and evaluation of various environmental noise regulations in the United States. Jeff D. Szymanski and W. Brent Ferren (Black and Veatch Corp., 11401 Lamar Ave., Overland Park, KS 66211, szymanskijd@bv.com)

Environmental noise in the United States is often regulated at the city, county, and/or state level. A review of some representative urban, suburban, and rural regulations that provide objective noise limits was conducted. The sound level metrics, if specified, were reviewed for applicability to various noise sources. Ambient sound level data recorded over the last 10-15 years in urban, suburban, and rural areas of the United States were compared to the applicable limits, as well as to other criteria. Comparisons were evaluated for consistency and ambient sound levels were compared to USEPA guidelines in order to evaluate their relevance more than 30 years later. The regulations and guidelines reviewed were found to have varying degrees of applicability.

THURSDAY MORNING, 7 JUNE 2007

CANYON ROOM A, 10:20 A.M. TO 12:00 NOON

\section{Session 4aNSb}

\section{Noise, Engineering Acoustics, and Signal Processing in Acoustics: Recent Advances in Active Noise Control I}

\author{
Scott D. Sommerfeldt, Cochair \\ Brigham Young Univ., Dept. of Physics and Astronomy, Provo, UT 84602-4673 \\ Kenneth A. Cunefare, Cochair \\ Georgia Inst. of Technology, School of Mechanical Engineering, Love Building, Atlanta, GA 30332-0405
}

\section{Invited Papers}

$10: 20$

4aNSb1. Recent developments in the use of structural actuators for decentralized active control. Stephen Elliott, Paolo Gardonio, Oliver Baumann, and Yoko Aoki (ISVR, Univ. of Southampton, Southampton, UK)

With an array of ideal, dual, collocated actuators and sensors, decentralized feedback controllers can not only be unconditionally stable, but their performance in reducing vibration and, hence, radiated sound, can approach that of a centralized LQG controller [W. P. Engles et al., JASA 119, 1487-1495 (2005)]. They also have a modular architecture that scales well, even for very large structures. This paper discusses some of the problems that arise in practice when proof-mass or piezoelectric transducers are used instead of ideal force or moment-pair actuators in such decentralized controllers. Proof-mass actuators are attractive when generating significant forces, but their natural frequency must be well below the first structural resonance frequency for stable operation. Even when this is achieved, impulsive forces due to the actuators hitting their end-stops have recently been shown to potentially be an additional source of instability. Piezoceramic actuators can give significant control in thinner structures, but as well as controlling out-of-plane vibration, they also create in-plane motion in the structure that can couple into closely located strain sensors causing instability in feedback controllers. Novel arrangements of such transducers will be described in which the effect of in-plane coupling is minimized, thus significantly improving their performance.

10:40

4aNSb2. Estimating the number of uncorrelated disturbance sources in structural systems. Suk-Min Moon (Adaptive Technologies, Inc., Blacksburg, VA 24060), Leslie Fowler (CSA Eng., Inc., Albuquerque, NM 87123), and Robert Clark (Duke Univ., Dept. of Mech. Eng. and Mater. Sci, Box 90300, Durham, NC 27708-0300)

The number of input signals used as reference inputs for feedforward control applications is limited due to cost, computational burden, input processing capability, and installation constraints. Identifying an optimal subset of reference sensors from a larger set capable of conveying the dynamics important in the performance path can result in greater performance with reduced complexity and order in the active control system. However, before determining an appropriate subset of sensors, the number of exogenous distur- 
bance sources must be determined. Principal component analysis can be used to determine the number of uncorrelated disturbances acting on a structural system. Singular value decomposition of a covariance matrix of measured sensor signals is used to determine the number of independent disturbances present in the dynamic system. Limitations imposed by sample data length, path dynamics, and noise can limit the ability to resolve the number of exogenous disturbance sources. To estimate the number of secondary disturbance sources, a controller can be used to minimize the response due to the dominant signal source. It is then possible to identify the significant secondary sources. This can lead to improved estimation of the minimum number of reference sensors required for feedforward control in structural and structural acoustic systems.

11:00

4aNSb3. Singular value decomposition of plant matrix in active noise and vibration-some examples. Alain Berry, Yann Pasco, and Philippe-Aubert Gauthier (GAUS, Dept. of Mech. Eng., Univ. de Sherbrooke, Sherbrooke, QC, J1K 2R1, Canada)

In active noise and vibration control problems that involve many secondary sources and error sensors, the active control performance is largely related to the conditioning of the plant matrix (formed by the transfer functions between individual secondary sources and error sensors). The principal component transformation (or singular value decomposition) of the plant matrix is an interesting tool to extract dominant secondary paths and limit control efforts. Furthermore, it can be used in a feedforward LMS controller to prevent slow convergence due to ill-conditioning of the plant matrix, and adjust the convergence rate of individual system modes. This approach is discussed through two different applications: (1) the multi-harmonic active structural acoustic control of a helicopter main transmission noise using piezoceramic actuators; (2) the broadband, adaptive sound field synthesis using multiple reproduction sources. It is shown that the approach allows decreasing the required signal processing and limiting the magnitude of the control inputs. Furthermore, in the case of sound field reproduction, it allows a very elegant and insightful interpretation in terms of controlling independent radiation modes.

11:20

4aNSb4. Negative impedance and vibration control of beams and plates. Kenneth A. Cunefare (Woodruff School of Mech. Eng., The Georgia Inst. of Technol., Atlanta, GA 30332-0405, ken.cunefare@me.gatech.edu)

The use of piezoelectric actuators for noise and vibration control purposes is well established. Recently, however, the use of negative impedance circuits for the control of such actuators has received increasing attention. This paper considers the fundamental implications of negative impedance control when used in the context of active vibration suppression on beams and plates. It is shown that action of the negative impedance control may be related to the minimization of the reactive power delivered into the structure, thereby minimizing the standing wave field. If one takes the perspective that the vibration field on a structure is composed of direct and reverberant components, then minimization of the reactive power input leads to attenuation of the reverberant component. The reverberant response is global, such that its minimization leads to global vibration minimization.

11:40

4aNSb5. Active noise control for double glazing. Christian Carme (TechnoFirst, Parc Industriel de Napollon, 48, ave des Templiers, 13676 Aubagne Cedex, France)

Implementation of ANC on double glazing windows has been investigated through controlling the volume of the air gap between the two window panes. In past work, the major problem was the location (generally in the middle of the glass plane) and/or efficiency of the transducer generating the counter noise. A new transducer (called strip-transducer) overcomes this problem by locating the loudspeaker system in the window frame. The transducer consists of a beam like rigid membrane (less than $20 \mathrm{~mm}$ width and $500 \mathrm{~mm}$ length) attached to a longitudinal voice coil suspended in the magnetic field generated by a set of magnets located in a metallic U-shaped profile. These strip-transducers are driven by a local ANC control loop, the reference sensor being either a microphone or an accelerometer located in the emitting side and the error microphone being located on the receiving side. The control loop algorithm is based on TechnoFirsts patented cloverleaf filter, allowing a faster response than traditional X-LMS algorithms. The transducer design uses the concept of tuned membrane resonator (TMR) to reduce the drive energy needed. A prototype using this new concept is under experimentation and has shown very encouraging results. 


\title{
Session 4aPA
}

\section{Physical Acoustics: Nonlinear and Linear Seismology and Time Reversal in Solids}

\author{
Brian E. Anderson, Chair \\ Los Alamos National Lab., Geophysics Group, Los Alamos, NM 87545
}

Chair's Introduction-8:30

\section{Contributed Papers}

$8: 35$

4aPA1. Nonlinear oscillations of an object buried in soil. Evgenia A. Zabolotskaya, Yurii A. Ilinskii, and Mark F. Hamilton (Appl. Res. Labs., Univ. of Texas, P.O. Box 8029, Austin, TX 78713-8029, hamilton@mail.utexas.edu)

A model is presented for nonlinear oscillations of an object buried in soil. The derivation is based on Lagrangian mechanics. The stress-free boundary condition on the surface of the ground is satisfied by introducing an image source pulsating in antiphase with the buried object. An equation of Rayleigh-Plesset form is obtained that describes the nonlinear volume oscillations in the elastic medium. In the linear approximation, the model yields an analytic expression for the resonance frequency. When elasticity of the soil is ignored, or when its elastic properties are presumed independent of depth, the resonance frequency decreases as burial depth is increased, tending toward a limiting value at saturation. When the shear modulus of the soil is permitted to increase with depth, the resonance frequency no longer saturates but instead increases with depth after passing through a minimum value. Perturbation theory is used to estimate weakly nonlinear effects. Attention is focused on second-harmonic and difference-frequency generation. [Work supported by ONR.]

\section{8:50}

4aPA2. Seismo-acoustic antivehicular landmine detection using phase data. Bill SchneckIII and Brad Libbey (Army RDECOM CERDEC NVESD, 10221 Burbeck Rd., Ft. Belvoir, VA 22060)

Landmine detection is tactically important for main supply route clearance in counter-insurgency operations. Seismo-acoustic detection is one of multiple technologies being pursued by the Army in an effort to address this need. The experiments to be presented were designed to address the use of phase data for landmine detection. These experiments are, in large part, a replication of the efforts by T Wang et al. [Proc. SPIE 5415, 70-79 (2004)]. In the present work, phase and magnitude data are used to detect three targets: a V-S 1.6 anti-vehicular mine (easy target), an M-19 antitank mine (difficult target), and a resonant plate (very easy control). These were tested in four situations: free space, flush bury, 1 bury, and 2 bury. The soil surface velocity was measured using an array of four laser Doppler vibrometers, and the acoustic pressure used for excitation was recorded with a microphone. In certain instances, the relative phase between the surface vibration and acoustic pressure directly above the mine was different from soil alone.

\section{9:05}

4aPA3. Nonlinear acoustic landmine detection: Comparison of tuning curve experiments profiling on and off the target. Sean A. Genis and Murray S. Korman (Dept. of Phys., U. S. Naval Acad., Annapolis, MD 21402)

Recent experiments [Proc. SPIE 6217, 62170Y, Detection and Remediation Technologies for Mines and Minelike Targets XI; J.T. Broach, R.S. Harmon, J.H. Holloway, Jr.; Eds. (2006)] have shown that the soil vibrating over a drum-like acrylic landmine simulant is more nonlinear on the target than well off (due to soil alone). Here, a Laser Doppler Vibrometer (LDV) is configured to scan laterally across the buried simulant. The 10 $\mathrm{cm}$ diameter, $5 \mathrm{~cm}$ high rigid simulant has a flexible clamped $0.8 \mathrm{~mm}$ thick top plate and is buried $4 \mathrm{~cm}$ deep in dry, sifted masonry sand in a rigid soil container placed inside the anechoic chamber. At each location the sand is subjected to a series of increasing amplitude acoustic pressure level sweeps (across a 50-200 Hz bandwidth) that includes the resonant behavior. The soil surface particle velocity vs. frequency tuning curves, from locations "off target" show relatively small nonlinear effects, while those from locations "on target" exhibit clearly identifiable backbone (peak amplitude vs. corresponding resonant frequency) curves that exhibit softening. Profiles of the backbone slope vs. position indicate an extremely large contrast ratio between "on" and "off" locations compared to profiles of surface vibration obtained near resonance. [Work supported by ONR.]

\section{9:20}

4aPA4. Nonlinear time reversal acoustic method of land mine detection: Experiment and modeling. Alexander Sutin (Artann Labs., West Trenton/Stevens Inst. of Technol., Hoboken, NJ), Brad Libbey (U.S. Army RDECOM CERDEC NVESD, Fort Belvoir, VA 22060), and Armen Sarvazyan (Artann Labs., West Trenton, NJ 08618)

The nonlinear vibration response of land mines is an effective detection clue. This response can be measured by application of two frequency acoustic waves producing a vibration spectrum above the mine that is different from its surrounding. As an alternative to this approach, this work investigates time reversal techniques as a method of acoustically exciting nonlinear mine vibrations over a broad frequency range. The phaseinversion method used here requires two short broadband time-reversed focused signals with opposite sign, i.e. phase inverted. The focused signal and the inverted focused signal are broadcast sequentially and the responses are summed in post processing. This cancels the linear response leaving the nonlinear component. A theoretical model has been developed that treats the mine stiffness as a nonlinear spring. It predicts that the dominant nonlinear frequencies correspond to the second harmonics of the mine's primary resonance. Experiments were conducted using six loudspeakers in a box placed directly over a mine. The measured responses confirm most of the model predictions, and illustrate differences between the soil and mine nonlinearities. Work was supported by the U.S. Army RDECOM CERDEC Night Vision and Electronic Sensors Directorate

9:35

4aPA5. Prediction of seismic pulses from an outdoor explosive source. Shahram Taherzadeh (Faculty of Technol., The Open Univ., Milton Keynes, MK7 6AA, UK) and Keith Attenborough (The Univ. of Hull, Hull, HU6 7RX, UK)

Near-surface layering of ground soil can influence propagation of seismic pulses originating from above-surface sources. Furthermore, in a cold climate snow and frozen ground can add to this layering effect. A numerical model called Fast Field Program for Layered Air Ground Systems (FFLAGS), developed originally for continuous sound sources above a porous elastic ground, is used to predict radial and vertical seismic signals from an above ground explosive charge recorded by a geophone. Simul- 
taneous acoustic air pressure recording of the same charge is used to make a prediction of the source pulse shape. This procedure produces an effective linear source. In other words, nonlinear effects are assumed to be small at the ranges of interest here. Subsequently, suitable optimization methods were used to predict a set of best fit parameters for the nearsurface ground structure. [Work supported in part by US Army through its European Research Office.]

\section{9:50}

4aPA6. Angle of incidence seismic/acoustic ratios during wet and dry ground conditions. Chris A. McNeill (Dept. of Phys. and Astron., 108 Lewis Hall, The Univ. of Mississippi, P.O. Box 1848, University, MS 38677) and James M. Sabatier (The Univ. of Mississippi, University, MS 38677)

Modeling the out-of-plane seismic-to-acoustic (S/A) transfer function shows frequency and angular dependences. The amplitude, frequency, and angle of incidence analysis shows rapid shifts in the frequency locations of the transfer function maxima as the angle of incidence of the sound source becomes greater than critical angles for the deeper soil layers. Outdoor measurements of the transfer function were conducted to verify this critical angle effect. These measurements used a suspended loudspeaker as a source and vertical and horizontal component geophones as receivers to measure the angular dependence of the in- and out-plane S/A coupling ratio during wet ground conditions. The calculated S/A ratio using sound speeds and layer depth collected during dry conditions revealed critical angle effects at the test site that are similar to those in the literature. However, the S/A ratio measurements during wet conditions did not exhibit such effects. Modeling the ground as a low speed wet soil layer over a fast bottom with water table sound speeds agrees with the measured wet ground transfer function data. The wet ground condition properties will be verified and S/A ratio measurements will be made during the dry ground conditions to verify these hypotheses.

\section{0:05-10:20 Break}

\section{0:20}

4aPA7. A long-term field soil survey by acoustic techniques: Part I. Linear acoustics. Zhiqu Lu (Natl. Ctr. for Physical Acoust., The Univ. of Mississippi, University, MS 38677)

Soils are complicated, multiphase granular materials; their behavior is determined by the discrete nature of these media, external and interparticle forces, interconnected porosity, and multiphase conditions. In a real world, the properties of field soils change ceaselessly due to seasonal and climate effects. It is generally difficult or impossible to simulate field soil conditions in the lab. To study and monitor the evolution process of soil properties, acoustic techniques are invoked for a long-term field soil survey. In this study, an acoustic probe with five acoustic transducers is inserted into the ground with minimum disturbance of soils. At different depths, sound speed, temperature, moisture, soil suction, and rain precipitation are recorded continuously. It is found that temperature has a negligible effect on the sound speed, moisture plays a minor role, and soil suction is the predominant factor governing the sound speed of the shallow field soil. Terzaghi's principle of effective stress is brought to explain the observations.

\section{0:35}

4aPA8. A long-term field soil survey by acoustic techniques: Part II. Nonlinear acoustics. Zhiqu Lu (Natl. Ctr. for Physical Acoust., The Univ. of Mississippi, University, MS 38677)

Soils are nonelastic and nonclassic nonlinear materials. They present loading-history-dependent, end-point memory, and hysteretic properties, which distinguish themselves from elastic materials such as intact crystal and fluids. Based on recent development in nonclassic nonlinear acoustic theory, a phase shift method is used to determine the hysteretic nonlinearity parameter of a field soil. In this method, the phase shift between the transmitter and receiver caused by increasing the sound level is measured and used to curve fit the equation of state that takes into account hysteresis, and to calculate the nonlinearity parameter. In a long-term survey, the hysteretic nonlinearity parameter, sound speed, temperature, moisture, soil suction, and rain precipitation are measured and their relations are studied. It is found that the nonlinearity parameter is much more sensitive than sound speed to the climate change. Soil suction is the predominant factor that affects the nonlinearity parameter. Unlike sound speed, the hysteretic nonlinearity parameter is sensitive to the variation of temperature.

10:50

4aPA9. Complex source imaging using time-reversal (TR): experimental studies of spatial and temporal resolution limits. Brian E. Anderson, Paul A. Johnson, T. J. Ulrich, Michele Griffa (Los Alamos Natl. Lab., Geophys. Group, MS D443, Los Alamos, NM 87545, bea@lanl.gov), James A. Ten Cate, and Lianjie Huang (Los Alamos Natl. Lab., Los Alamos, NM 87545)

Large earthquakes are composed of a complex succession of slip events that are nearly indistinguishable on a seismogram. The question, how does an earthquake work? remains largely unsolved. The slip events on the fault plane(s) generally take place at different spatial locations and at different times. TR wave physics can be advantageously exploited to recreate, from measured signals, a spatially and/or temporally complex sound/seismic source. An experimental study is conducted to determine the spatial and temporal resolution limitations in imaging a complex source in solids, as part of our goal to understand earthquake source complexity. TR experiments are conducted on solid blocks of different materials, such as Berea sandstone and aluminum. Arrays of piezoelectric transducers are bonded to the samples for the creation of complex spatialtemporal sources, as well as to record signals. The experimental spatial and temporal resolution limits for complex source imaging will be presented as a function of material physical characteristics (e.g., Q, modulus), as well as source signal characteristics such as pulse width, frequency and repetition rate. [This work was supported by Institutional Support (LDRD) at Los Alamos National Laboratory.]

\section{1:05}

4aPA10. Nonlinear dynamics and time reversed acoustic imaging in damaged solids. T. J. Ulrich, Brian E. Anderson, Paul A. Johnson (Los Alamos Natl. Lab., Geophys. Group, M.S. D443, Los Alamos, NM 87545, tju@lanl.gov), Alexander M. Sutin (Stevens Inst. of Technol., Hoboken, NJ 07030), and Robert A. Guyer (Univ. of Massachusetts, Amherst, MA 01003)

Our ultimate goal is locating and imaging nonlinear scatterers in solids (e.g., cracks) without a priori knowledge of their existence. Toward that goal, two methods have been devised that combine the spatial and temporal focusing abilities of TRA with nonlinear elastic wave spectroscopy's (NEWS) sensitivity to mechanical damage. The first method uses TRA to create large amplitude signals and induce a nonlinear response in a highly localized region on a sample surface. Repeating the process in a step-scan approach provides the means to image the sample surface/near-surface with high resolution, distinguishing nonlinear features (cracks) from the linear background (undamaged material). The second method takes advantage of TRA to focus acoustic energy from two input frequencies onto an unknown, but nonlinear, scattering source, creating nonlinear wave modulation. The time reversal mirror (TRM) array records the primary and scattering source waveforms. Filtering the recorded signals for a modulation sideband, time reversing, and rebroadcasting through the TRM focuses the filtered signal onto the source of the modulation-the crack. Experimental results demonstrating each technique will be presented. [This work was supported by Institutional Support (LDRD) at Los Alamos National Laboratory.] 


\title{
Session 4aPP
}

\section{Psychological and Physiological Acoustics: Topics and Methods in Environmental Sound Research}

\author{
Valeriy Shafiro, Cochair \\ Rush Univ. Medical Center, Communication Disorders and Sciences, 1653 W. Congress Parkway, Chicago, IL 60612 \\ Brian M. Gygi, Cochair \\ Acoustic Research Inst., 150 Muir Rd., 151-I, Martinez, CA 94553
}

Chair's Introduction-8:30

Invited Papers

8:35

4aPP1. Environmental sound research as it stands today. Brian Gygi (East Bay Inst. for Res. and Education, 150 Muir Rd., Martinez, CA 94553) and Valeriy Shafiro (Rush Univ. Medical Ctr., Chicago, IL)

Environmental sound research is still in its beginning stages, although in recent years a body of research has started to accumulate, both on the perception of environmental sounds themselves and on their practical applications in other areas of auditory research and cognitive science. However, several important issues remain unresolved, such as methodologies for environmental sound research, how to coordinate research efforts in disparate areas of environmental sound study, and how best to integrate environmental sound research into the larger body of hearing research. These issues will be discussed along with some of the current and future practical applications of environmental sounds, such as in assistive listening devices and automatic sound source recognition.

\section{$8: 55$}

4aPP2. Multiple means of conveying information through sound: Comparisons of environmental sounds and spoken language processing using converging methodologies. Frederic Dick, Robert Leech (Birkbeck College, Univ. of London, Malet St., London, WC1E 7JX, UK), Alycia Cummings, Arielle Borovsky (UC-San Diego, La Jolla, CA), and Ayse Pinar Saygin (UCL, Queen Square, London, UK)

Environmental sounds are increasingly viewed as an attractive nonlinguistic analog for studying meaningful speech in that they can convey referential—or at least associative—information about objects, scenes, and events that unfold over time. However, environmental sounds also differ significantly from speech along other perceptual and informational parameters. These cross-domain similarities and differences have proved useful in uncovering the perceptual and cognitive divisions of labor in the developing and mature brain. Our group has directly compared environmental sound and spoken language understanding in a series of behavioral and neuroimaging studies with infants, typically and atypically developing children, healthy adults, and aphasic patients. In general, our results suggest that environmental sounds and language share many of the same processing and neural resources over the lifespan. [This research is supported by the National Institutes of Health and the Medical Research Council.]

9:15

4aPP3. Effects of training on the perception of environmental sounds with limited spectral resolution. Valeriy Shafiro (Dept. Commun. Disord. \& Sci., Rush Univ. Medical Ctr., Chicago, IL 60612, valeriy_shafiro@ rush.edu)

Environmental sounds play an important role in maintaining listeners' awareness of the sound sources in the environment. They can warn of potential dangers (e.g., alarms, collisions) or provide aesthetic satisfaction (e.g., babbling brook, bird song). Although research indicates that decreased spectral resolution has a negative effect on environmental sound identification, little is known about perceptual adaptation to spectrally sparse environmental sounds. This project investigated changes in the ability to identify spectrally smeared environmental sounds in response to training. In a pretest-posttest design, seven naive normal-hearing listeners, identified the spectrally smeared environmental sounds obtained with a four-channel noise-based vocoder. Five training sessions were administered between the pretest and the posttest. Overall mean identification performance improved from $33 \%$ on the pretest to $63 \%$ on the posttest, with the largest improvement of $86 \%$ obtained for the sound tokens used during training. Increases in identification accuracy were also observed for alternative tokens of the training sounds ( $36 \%$ difference) and sound sources not included in the training set (31\% difference). Among those, the greatest improvement was found among sounds with distinct envelope patterns. The implications of these findings for perceptual learning and rehabilitation programs for cochlear implant patients will be discussed. 
4aPP4. Perceptual and acoustic correlates of perceptual structures of environmental sounds. Terri Bonebright (Dept. of Psych., 7 E. Larabee St., DePauw Univ., Greencastle, IN 46135, tbone@ depauw.edu) and Michael Nees (Georgia Inst. of Technol., Atlanta, GA 30332)

The present study was designed to provide insight into the perceptual structure of everyday sounds and how that structure relates to the external acoustic signal. Two large stimulus sets of 74 sounds each were used to gather sorting data, attribute ratings, and basic acoustic measurements for analysis using multidimensional scaling solutions (MDS). Correlations between and among the acoustic measurements and attribute ratings were as expected. The resulting MDS solutions with regressed vectors for attribute ratings and acoustic measurements revealed a well-defined three-dimensional perceptual structure for both stimulus sets. Similarities and differences in the scaling solutions will be discussed. Information about perceptual structure from such studies can be used by researchers to increase basic knowledge about how people perceive the relationships among everyday sounds as well as by designers of virtual reality environments to assist in developing algorithms for realistic synthesized sounds.

\section{9:55-10:10 Break}

\section{0:10}

4aPP5. Influence of auditory information on auditory-visual speed discrimination. Laurie M. Heller (Dept. of Cognit. and Linguistic Sci., Brown Univ., 190 Thayer St., Providence, RI 02912, Laurie_Heller@brown.edu)

Auditory information can exert a strong influence on multimodal perception. In the experiments described here, a video of a ball rolling in depth was paired with the sound of a ball rolling. The speed of the balls' roll in the video was independently varied from the speed of the balls' roll in the soundtrack. Total duration was held constant so that the auditory cues to speed were ongoing spectro-temporal features of the rolling sounds. In a 2IFC comparison, observers indicated which ball seemed to roll faster. In half of the trials the near-threshold visual and auditory speed information was consistent, e.g., both the video and sound sped up between the first and second interval. Performance improved as much as would be predicted by a linear model. On the other half of the trials in which the auditory and visual information were inconsistent, performance declined accordingly. [Work supported by NSF.]

\section{0:30}

4aPP6. Statistical modeling of natural auditory source event perception. Richard Pastore, Jesse Flint, and Jeremy Gaston (Dept. of Psych., Binghamton Univ., Binghamton, NY)

Challenges to understanding auditory source perception can be understood by contrast with classic psychophysics. Psychophysics studies the relationship between properties of sound and perception; by mapping changes in a single perceptual dimension as a function of controlled, systematic variation in a single acoustic property. This severely limits the degrees of freedom for both the listeners strategy and the investigators interpretation of the complex, often nonlinear, sound-perception relationships. Source perception investigation not only adds the original event as an analysis stage, but also multiple levels of complexity to every component in the analysis stages. Natural source events are complex and, although acoustic properties must reflect source event structure, variation in many acoustic dimensions of the produced sounds is determined both separately and interactively by attributes that vary with all source properties, including the one(s) investigated. Furthermore, physical acoustic dimensions seldom map directly to perceptual dimensions. Finally, listeners have many degrees of freedom in assigning importance weighting to the multitude of sound attributes, differ in their weightings, and are seldom optimal. The current paper discusses statistical modeling approaches for developing a systematic understanding of the relationships among the source-sound-perception components to event perception. [Work supported by NSF grant BCS-0213666.]

\section{0:50}

4aPP7. The object of perception in impacted sound sources: The role of perceptual accessibility and information accuracy. Bruno L. Giordano, Stephen McAdams (CIRMMT, Schulich School of Music, McGill Univ., 555 Sherbrooke W., Montreal, QC, H3A 1E3 Canada), and Davide Rocchesso (Univ. of Verona, 37134, Verona, Italy)

Two independent principles could potentially govern integration of information in the perception of a sound source. First, higher perceptual weights should be given to acoustical parameters that specify accurately and unambiguously a sound source property. Second, lower perceptual weights should be given to acoustical parameters related to less perceptually accessible information (e.g., impaired discrimination). The relevance of these principles to source perception was investigated. Hardness perception was investigated for the two objects whose interaction generates an impact sound: a highly damped hammer and a freely vibrating sounding object. Acoustical analyses identified unambiguous and relatively accurate acoustical specifiers of the impacted sound source. A first experiment assessed the ability of a listener to learn to discriminate hammer or sounding object hardness, providing a measure of the perceptual accessibility of the two source properties. A second experiment investigated hardness estimation in trained and untrained listeners, providing quantification of the relative perceptual relevance of the informational sources. A final experiment conducted on simulated sound sources further characterized weighting profiles in untrained listeners, decorrelating otherwise covarying source properties. Results concerning both mechanical and acoustical correlates suggest a relevance of both principles.

11:10-11:40

Panel Discussion 


\title{
Session 4aSC
}

\section{Speech Communication: Cross-Linguistic Consonant Production and Perception (Poster Session)}

\author{
Shawn L. Nissen, Cochair \\ Brigham Young Univ., 138 TLRB, 1190 N. 900 E, Provo, UT 84602 \\ Bruce L. Smith, Cochair \\ Univ. of Utah, Dept. of Communication Disorders, 390 S. 1530 E., Salt Lake City, UT 84112-0252
}

\section{Contributed Papers}

\begin{abstract}
All posters will be on display from 8:30 a.m. to 11:30 a.m. To allow contributors an opportunity to see other posters, contributors of odd-numbered papers will be at their posters from 8:30 a.m. to 10:00 a.m. and contributors of even-numbered papers will be at their posters from 10:00 a.m. to 11:30 a.m.
\end{abstract}

\begin{abstract}
4aSC1. Changes in acoustic characteristics of / $r$ / following production training using electropalatography (EPG). Anna Schmidt (School of Speech Path. \& Aud., Kent State Univ., Kent, OH, 44240, aschmidt@kent.edu)
\end{abstract}

Visual feedback using electropalatography (EPG) is an effective method for changing production of speech sounds in children. Differences in tongue placement and general acceptability of production have been demonstrated with EPG training for tongue to palate contact. Little is known, however, about changes in the spectral characteristics of speech sounds following training. The current study examined pre and post EPG patterns for $/ \mathrm{r} /$ in prevocalic, intervocalic, and postvocalic positions in sentences with five weeks of intensive EPG training. In particular, spectral characteristics of $/ \mathrm{r} /$ were examined for differences pre and post treatment. Subjects were four children (aged 9-10). These children exhibited distorted /r/ despite several years of traditional speech therapy but learned to produce perceptually acceptable /r/ with EPG. Spectral characteristics for /r/ productions in the same sentences by four typically developing children were used for comparison. Differences will be discussed in terms of acoustic characteristics as well as changes in tongue position.

4aSC2. Whistly fricatives: MRI and acoustic data. Christine $\mathrm{H}$. Shadle, Michael Proctor, and Khalil Iskarous (Haskins Labs., 300 George St., New Haven, CT 06511, shadle@ haskins.yale.edu)

Nonlinear effects in airflow during fricative production can lead to a whistly acoustic quality, which is used contrastively in a few languages and can occur due to dental appliances, but also often occurs in fluent speech. In a magnetic resonance imaging (MRI) study of American English fricatives produced by five subjects, it was noted that some of the subjects produced whistly fricatives. Articulatory and acoustic analyses will be presented to argue that small articulatory changes can produce significantly different acoustic results within the same segment class for a single subject. Lip rounding and presence of a sublingual cavity can contribute to whistling, but a single subject can produce whistly or normal frication if either of these factors is present or absent. Possibly different whistle mechanisms (edge versus hole tone) could account for some of these seeming anomalies. Strident fricatives are therefore an example of a class of speech sounds where small articulatory differences can lead to significant acoustic differences. [Research supported by NIH Grant No. NIDCD R01 DC 006705.]
4aSC3. Fourth formant dip as a correlate of American English flaps. Mary Dungan, Karen Morian, Benjamin V. Tucker, and Natasha Warner (Univ. of Arizona, Box 210028, Tucson, AZ 85721-0028)

Unlike the lower formant frequencies, the fourth formant frequency (F4) is rarely an important acoustic correlate of speech. This study shows evidence of a depression in F4 for some tokens of the American English flap. F4 may be lowered during American English /r/ (Espy-Wilson et al., 2000) and during retroflex sounds of some languages (e.g., Pima, as found by Avelino and Kim, 2002), but other cues are likely to be more prominent for such sounds. The current project uses a corpus of acoustic measurements of flapped English /t, d/ (e.g., pretty, order) and shows an inconsistent but large drop in F4 at the flap consonant. This occurs predominantly near an $/ \mathrm{r} /$, but the F4 drop is timed to the flap, not the $/ \mathrm{r} /$. In some tokens, this F4 drop occurs even if the flap is reduced to an approximant, leaving few other acoustic cues to its presence. The F4 drop likely reflects a sublingual cavity resonance caused by an interaction of the tongue positions for $/ \mathrm{r} /$ and for a flap. This study presents results on the distribution, frequency of occurrence, and magnitude of the F4 drop. Future research will investigate the perceptual role of the F4 drop.

4aSC4. Incomplete neutralization of $/ t /$ and $/ d /$ in flapping environments: An analysis of the North Midland dialect of American English. Wendy J. Herd (Dept. of Linguist., Univ. of Kansas, 1541 Lilac Ln., Blake Hall, Rm. 427, Lawrence, KS 66044-3177)

This paper presents an acoustic study of alveolar flaps in American English as produced by 20 speakers of a North Midland dialect spoken in the Kansas area. Participants read three lists of 164 monosyllabic and disyllabic words and nonwords containing word-final and medial /t/ and $/ \mathrm{d} /$. Vowel duration differences in monosyllabic and disyllabic tokens and consonant duration differences in monosyllabic tokens duplicated previous findings in that vowels preceding /d/ were significantly longer than those preceding $/ t /$ and in that the average duration of $/ t /$ was significantly longer than that of $/ \mathrm{d} /$. However, flap duration measured significantly longer for /d/-flaps than /t/-flaps. When analyzing flap frequency, it was also discovered that females flapped significantly more often than males and that participants were more likely to flap when they were less aware of the contrast between $/ \mathrm{t} /$ and $/ \mathrm{d} /$. While previous research reported that monomorphemic and high-frequency words are more likely to be flapped than bimorphemic and low-frequency words, neither word frequency nor morphological complexity affected flap frequency in the present study. Flap 
frequency was based on a method of distinguishing flapped from unflapped stops on a speaker-by-speaker basis, a more accurate method than that used in previous studies.

4aSC5. A motor differentiation model for liquid substitutions in children's speech. Bryan Gick (Dept. of Linguist., Univ. of British Columbia, E270-1866 Main Mall, Vancouver, BC V6T 1Z1, Canada)

Studies of lip-jaw coordination in children have shown a lack of motor differentiation between anatomically coupled articulators in young childrens speech [Green \& al. 2000, JSLHR 43: 239-255]. A model is described in which children contending with their developing motor systems generally strive to reduce the degrees of freedom of complex anatomical structures (e.g., the tongue). The claim is pursued that segmental substitutions (e.g., /w/ replacing /r/ or / / / ) are the result of specific compensation strategies which aim to simplify the complexity of the articulatory task. The proposal that gestural simplification may dictate substitution strategies for liquid consonants has been suggested previously [Studdert-Kennedy \& Goldstein 2003, Language Evolution, Oxford U. Pr. 235-254]. It is proposed here that gestural simplification may be achieved via one of two basic mechanisms: gestural omission and stiffening (and hence merger), and that these two mechanisms account for all of the commonly attested substitutions for English /r/ and /1/. Supporting data are presented from ultrasound studies of: postvocalic /r/ production of an 11-month-old female speaker of English, liquid production of a group of 3-5-year-old speakers of English, and liquid production and substitutions in the speech of adolescent speakers of English with speech and hearing disorders.

4aSC6. Acoustic cues of "retroflex" and "bunched" American English rhotic sound. Xinhui Zhou, Carol Espy-Wilson (Dept. of Elec. and Computer Eng., Univ. of Maryland, College Park, MD 20742), Mark Tiede (Haskins Labs, New Haven, CT 06511), and Suzanne Boyce (Univ. of Cincinnati, Cincinnati, OH 45202)

The North American rhotic liquid has two well-known and maximally distinct articulatory variants, the classic retroflex tongue posture and the classic bunched tongue posture. The evidence for acoustic difference between them is reexamined using magnetic resonance images of the vocal tracts from two similar-sized subjects with different tongue postures of sustained /r/. Three-dimensional finite element analysis is performed to investigate the acoustic wave propagation property inside the vocal tract, the acoustic response, and the area function extraction based on pressure isosurfaces. Sensitivity functions are studied for formant-cavity affiliation. It is revealed that these two variants have similar patterns of F1-F3 and zero frequency. However, the retroflex variant is predicted to have a larger difference between F4 and F5 than the bunched one (1400 Hz versus 700 $\mathrm{Hz}$ ). This difference can be explained by the geometric differences between them, in particular, the shorter, narrower, and more forward palatal constriction of the retroflex variant. In addition, the predicted formant pattern is confirmed by measurement from acoustic data of sustained $/ \mathrm{r} /$ and $/ \mathrm{r} /$ in nonsense words from several additional subjects. These results may prove to be useful to researchers in speech motor control, speech pathology, speaker verification, speech recognition, and speech synthesis. [Research supported by NIH.]

4aSC7. Talker-specific contextual influences on voice-onset-time. Rachel M. Theodore, Joanne L. Miller, and David DeSteno (Dept. of Psych.-125 NI, Northeastern Univ., 360 Huntington Ave., Boston, MA, 02115-5000)

Research has shown robust contextual influences on voice-onset-time (VOT) in speech production. The current work examines talker-specificity for two such cases: speaking rate (VOT increases as syllable duration increases) and place of articulation (VOT increases as place moves from anterior to posterior position). Tokens of /pi/ (labial) and /ki/ (velar) were elicited from talkers across a range of rates. VOT and syllable duration were measured for each token. For each talker, separate labial and velar linear functions relating VOT to syllable duration were calculated. Ongoing analyses indicate that (1) for both the labial and velar functions there is significant variability across talkers' slopes [see also Theodore et al., J. Acoust. Soc. Am. 120, 3293 (2006)], but there is no significant variability in the difference between labial and velar slopes for a given talker. Thus the effect of speaking rate is talker-specific, and stable across place of articulation. (2) For each talker, the velar intercept is located at a longer VOT than the labial intercept, with significant variability in the magnitude of displacement across talkers. Thus the effect of place is also talker specific. These findings support the view that phonetic properties of speech include talker-specific contextual influences. [Work supported by NIH/ NIDCD.]

4aSC8. Rate normalization of closure duration influences on an assimilation context effect. David Gow (Massachusetts General Hospital, CPZ-S340, 175 Cambridge St., Boston, MA 02114 and Salem State College, 352 Lafayette St., Salem, MA 01970) and Claire WinchellManning (Salem State College, Salem, MA 01970)

Variation in speech rate influences the structure and distribution of phonetic cues in a variety of ways. For example, a large body of work demonstrates that the rate of speech and nonspeech precursors influences the interpretation of temporal cues associated with phoneme categorization. Changes in speaking rate also influence the absolute size of the temporal window over which multiple cues to the identity of a segment may be distributed. This has potentially significant implications for the perception of assimilated speech in which feature cues from adjacent segments interact to produce bidirectional context effects. These implications were explored in a series of mousetracking experiments involving a nonnative gradient regressive voice assimilation process that is sensitive to the duration of a silent interval between the offset of a partially voiced wordfinal fricative and the onset of a following voiced or unvoiced stop. Manipulations of precursor rate interacted with assimilation context effects in the interpretation of an /ais/-/aiz/ continuum. These results are discussed in the context of competing interpretations of assimilation context effects and rate normalization. [Work supported by NIH R01DC3108.]

4aSC9. Consonant duration and vowel-to-vowel coarticulation in Japanese. Anders Lofqvist (Haskins Labs., 300 George St., New Haven, CT 06511, lofquist@haskins.yale.edu)

This study examined vowel-to-vowel coarticulation in sequences of vowel-bilabial consonant-vowel, where the duration of the oral closure for the consonant is varied for linguistic purposes. Native speakers of Japanese served as subjects. The linguistic material consisted of Japanese word pairs that only differed in the duration of the labial consonant, which was either long or short; the durational ratio of short and long consonants in Japanese is about 1:2. Recordings were made of lip and tongue movements using a magnetometer system. It was hypothesized that there would be greater vowel-to-vowel coarticulation in the context of a short consonant, since a long consonant would allow the tongue more time to move. The results do not show any strong support for this hypothesis, however. The reason is that Japanese speakers modulate the speed of the tongue movement between the two vowels according to consonant duration, making it faster during a short consonant and slower during a long consonant. [Work supported by NIH.]

4aSC10. Voiced geminates in Yamagata and Tokyo Japanese. Lynnika Butler (Univ. of Arizona, Douglass Bldg. Rm. 200E, Tucson, AZ 85721, lgbutler@email.arizona.edu)

Voiced geminate stops $(/ \mathrm{bb} /, / \mathrm{dd} /, / \mathrm{gg} /$, etc.) are cross-linguistically rare, due to aerodynamic constraints on voicing during prolonged closure of the vocal tract. Standard Tokyo Japanese prohibits voiced geminate stops in native and Chinese-borrowed vocabulary, but permits them in 
some recent loanwords (primarily English borrowings), where they reflect stress on the preceding syllable of the word in the originating language. (1)big-/biggu/ (2) headphone-/heddohoN/ Yachi-ben (YB) is a local subdialect of the northeastern (Tohoku) dialect of Japanese, which is characterized in part by voicing of most non-initial stops. Transcriptions of YB speech recorded in the 1970 s by Japans National Language Research Institute (NLRI) indicate a sizeable number of voiced geminate stops, most frequently /dd/ but also /gg/ (there are no /bb/tokens). In this study, voiced geminate stops from the NLRI recordings were compared with voiced geminate stops in English loanwords as pronounced by Tokyo Japanese (TJ) speakers. Closure duration, as well as duration and amplitude of closure voicing, were measured for both sets of data. Overall, voicing ratios (the percent of closure duration in which voicing was present) were found to be much longer in YB voiced geminates than in TJ voiced geminates.

4aSC11. Acoustic correlates of emphatic consonants in Arabic. Allard Jongman, Wendy Herd, and Mohammad Al-Masri (Linguist. Dept., Univ. of Kansas, 1541 Lilac Ln., Lawrence, KS 66044, jongman@ku.edu)

Emphasis is a distinctive feature of Semitic languages such as Arabic that refers to a group of consonants produced with a secondary constriction in the posterior vocal tract and a primary constriction typically in the dental/alveolar region. Initial findings of a detailed acoustic investigation of emphasis in Urban Jordanian Arabic will be presented. Eight adult speakers of Urban Jordanian Arabic (four males and four females) produced a list of minimal pairs of emphatic versus plain CVC words. The list

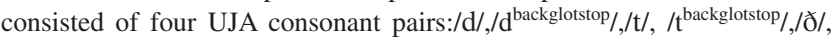

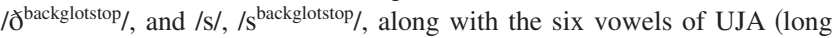
and short /i/, /a/, and /u/. Each target consonant occurred in both word-initial and word-final position. Results show consistently higher $\mathrm{F} 1$ and F3 values and lower F2 values for vowels in emphatic consonantal environments compared to vowels in plain environments. In addition, emphatic consonants have a lower spectral mean than their plain counterparts. Results from bisyllabic words will also be presented to evaluate the direction and extent of the spread of emphasis within the word. [Research supported by NSF.]

4aSC12. Production of Parisian stops word-initial stops in the vicinity of a voiceless stop. Nassima Abdelli-Beruh (Long Island Univ., C.W. Post Campus, 720 Northern Blvd., Brookville, NY 11548)

This study examines how French speakers produced stop voicing contrasts in the sentence répète CVC tout-de-suite. Durations (sentence, vowel before and after word-initial stops, closure voicing, VOT) and number of produced stop closures $(/ \mathrm{t} /, / \mathrm{C} /)$ were collected. There was a strong positive correlation between speaker's sentence durations and number of closure produced: Rapid speakers produced mostly one closure, whereas slow speakers generally produced two closures. One-closure and twoclosure productions were separately analyzed. In the two-closure productions, significant (longer voicing in the /t/ closure in anticipation of /bdg/ than /ptk/, longer closure voicing for $/ \mathrm{bdg} /$ than for $/ \mathrm{ptk} /$, longer vowel duration following /bdg/ than /ptk/) and nonsignificant voicing-related duration differences (e.g., closure) were found. Unlike in the two-closure productions, average closure duration was significantly longer before /ptk/ than before $/ \mathrm{bdg} /$, while there was no significant voicing-conditioned effect of the word-initial stop on the duration of the following vowel in the one-closure productions. As in the two-closure productions, average closure voicing duration remained significantly longer before /bdg/ than before /ptk/. Findings suggest that closure voicing remains the most salient cue to the voicing distinction irrespective of contextual and speaking rate variations.
4aSC13. A preliminary investigation of voice onset time associated with Turkish stops. Janine Sadaka (Dept. of Commun. Sci. and Disord., Long Island Univ., C.W. Post, 720 Northern Blvd., Brookville, NY 11548, janinesadaka@gmail.com) and Manwa L. Ng (The Univ. of Hong Kong, Sai Ying Pun, Hong Kong)

Voice onset time (VOT) refers to the timing between the release of a stop and the onset of the following vowel. Aspiration directly reflects the period of voicelessness following a stop release. Both VOT and aspiration are temporal measurements that are results of coordinated articulatory action. The present study will attempt to determine the relationship between VOT and aspiration, and VOT and voicelessness of Turkish stops. Comparison will also be made with those reported for English. Voicing differentiation in stops requires complex motor control. Thus, many speech disorders are associated with distorted VOT. Results will be used to develop a norm on VOT for Turkish stops. This will assist in evaluating speech disorders in Turkish-speaking individuals that involve motor control problems.

4aSC14. On the phonetics of Cypriot-Greek geminate plosives. Christiana Christodoulou (2111 Lower Mall, Univ. of British Columbia, Vancouver, BCV6T 1Z4, Canada)

This research provides evidence toward the existence of geminates in Cypriot Greek (hereinafter, $\mathrm{CyG}$ ). In the existing literature it is reported that the sole or primary cue to gemination, across languages, is closure duration. This phonetic study verifies that this cross-linguistic phonetic cue is also present in CyG. Toward this end, statistical analyses evidence highly significant durational differences in the voice onset time (VOT) of geminates versus singletons in CyG $[F(164)=p<0.0001]$. In fact it can safely be argued that VOT is the primary cue to gemination when it comes to $\mathrm{CyG}$ geminate plosives. This study contradicts previous claims [Arvaniti and Tserdanelis, 562 (2000); Arvaniti, 602 (1999b)] that argue closure duration to be the primary cue to gemination for $\mathrm{CyG}$ geminate stops. Furthermore, durational differences amongst the three voiceless plosives for both VOT and closure duration were highly evident. To the researchers' knowledge this is the first study on CyG stops that considers durational differences with respect to the place of articulation, not only for singletons but also for geminates. Preliminary statistical analysis suggests an effect of the vowel following the target segment, a fact that could facilitate in resolving the phonological representation of utterance initial geminates.

4aSC15. The effects of morphological boundaries and speech rate on geminate closures in Turkish. Molly Babel and Anne Pycha (Univ. of California, Berkeley, Berkeley, CA 94720-2650)

In Turkish, geminate consonants can occur in a word root and in derived positions across morphemes. In this experiment we explore closure duration and VOT in geminate consonants within roots, across root-suffix boundaries, suffix-suffix boundaries, and novel compound boundaries. Five $(3=$ male, $2=$ female) native speakers of Turkish were recorded producing 80 target and 20 filler words with an equal number of [tt], [kk], [11], and $[\mathrm{mm}]$ geminates in the four different morphological contexts named above. Subjects were instructed to produce each word in a carrier phrase three times. The wordlist was read twice by each talker. The first time subjects were asked to read at a slow, comfortable pace. The second time through, the list subjects were instructed to speak as quickly as possible without making mistakes. Consonant closure duration will be measured for the geminates. The voice onset time associated with $[\mathrm{tt}]$ and $[\mathrm{kk}]$ closures will also be measured. The results of the investigation have implications for theoretic phonological accounts of geminates. 
4aSC16. Processing of fine phonetic detail in American English flaps. Benjamin V. Tucker (Dept. of Linguist., Univ. of Arizona, P.O. Box 210028, Tucson, AZ 85721-0028)

Listeners are very good at recognizing casual, reduced speech in their native language. In day-to-day conversation acoustic information is often impoverished or reduced. Reduction in speech can occur in the form of deletion and/or change of segments, syllables, or words [Johnson (2004)]. This study explores differences in processing between reduced/ conversational speech and unreduced/careful speech. Two lexical decision studies, using reduced and unreduced pairs of the American English flap [Warner and Tucker (2007)], investigate whether processing differences exist between reduced and unreduced speech. The first study presented target words to listeners in isolation and the second study presented the same targets preceded by a reduced or unreduced frame sentence. The materials contained, among 260 items, 40 word medial /d/ phonemes and 40 word medial $/ \mathrm{g} /$ phonemes occurring in a flapping environment [Zue and Laferriere (1979)]; targets were produced in both reduced and unreduced form. These materials, spoken by a native American English speaker, were presented to 55 monolingual American English students from the University of Arizona. Listeners performed the lexical decision task for each item and response time and error rate was measured. Native English listeners process unreduced items more quickly than reduced items.
4aSC17. Nature versus nurture: Children's development and perception on syllable-final nasals in Taiwan Mandarin. Renee Hung (Grad. Inst. of English, Natl. Taiwan Normal Univ., No. 162, Sec. 1, HePing E. Rd, Taipei Taiwan 106, reneejh@hotmail.com) and Janice Fon (Grad. Inst. of Linguist., Natl. Taiwan Univ., Taipei, Taiwan 106)

In Hung and Fon's study (2006), it was found that elementary school children in Taiwan also tend to merge /əN/ with /ən/ as the way adults do, and the merging rates found in reading characters and sentences are higher than those in reading Zhuyinfuhao, a local phonetic system. However, while most adults and older elementary school children merge /in/ with /iN/, younger children have much lower merging rates for /i/ no matter under what condition. The first part of this study extends its investigation to preschoolers who have not officially learned Zhuyinfuhao. A picturenaming task was conducted with 20 kindergarten children. The results show that preschoolers have very similar performances with the adults' for both vowels. The second part of this study is a perception experiment. Sixty Grade- 2 and Grade- 4 students participated in the task, in which they were asked to write down the characters and phonetic symbols of the monosyllabic words they heard. Preliminary results indicate that these children are apt to treat /in/ and /əN/ words as homophones of /iN/ and /ən/ words, respectively, although such occurrences for /ə/ are lower than those for $/ \mathrm{i} /$.

THURSDAY MORNING, 7 JUNE 2007

GRAND BALLROOM C, 8:00 TO 11:50 A.M.

\title{
Session 4aSP
}

\section{Signal Processing in Acoustics, Acoustical Oceanography and Underwater Acoustics: Signal Processing and Uncertainty I}

\author{
Lee Culver, Cochair \\ Pennsylvania State Univ., Applied Research Lab., P. O. Box 30, State College, PA 16804-0030 \\ Ning Xiang, Cochair \\ Rensselaer Polytechnic Inst., Architecture, 100 8th St., Greene Bldg., Troy, NY 12180
}

Chair's Introduction-8:00

8:05

4aSP1. Sequential Bayesian signal processing: An overview. James Candy (Univ. of California, Lawrence Livermore Natl. Lab., P.O. Box 808, L-156, Livermore, CA 94551)

\begin{abstract}
Bayesian signal processing is concerned with the estimation of an underlying posterior distribution to extract statistics (inference) solving a variety of problems (signal enhancement, detection, parameter estimation, etc.). The technique simply employs Bayes rule to estimate the posterior using all of the data available at the time. Bayesian methods require approaches that capture all of the $a$ priori information available and are typically model-based employing mathematical representations of the embedded processes. An overview of Bayesian inference from batch to sequential processors is presented. Once the evolving Bayesian paradigm is established, simulation-based methods using sampling theory and Monte Carlo realizations are discussed. Here the usual limitations prevalent in classical processing algorithms (e.g., Kalman filters) are no longer a restriction to perform Bayesian inference. Next importance sampling methods are briefly discussed and shown how they can be extended to sequential solutions implemented using Markovian models as a natural evolution. With this in mind, the idea of a particle filter, which is a discrete representation of a probability distribution, is developed and shown how it can be implemented using sequential importance sampling/resampling methods. Finally, a set of applications is briefly discussed comparing the performance of the particle filter designs with classical implementations.
\end{abstract}

\section{8:35}

4aSP2. Bayesian inversion methods in ocean geoacoustics. Stan E. Dosso (School of Earth and Ocean Sci., Univ. of Victoria, Victoria, BC, Canada, sdosso@uvic.ca)

This paper describes a complete approach to the inversion of ocean acoustic data for environmental model parameters, which is also applicable to other inverse problems. Within a Bayesian formulation, the general solution is given by the posterior probability density (PPD) of the model parameters, which includes both data and prior information. Properties of the PPD, such as optimal parameter estimates, variance/covariance, inter-parameter correlations, and marginal probability distributions, are computed numeri- 
cally for nonlinear inverse problems using Markov-chain Monte Carlo (MCMC) importance sampling methods. Since the data uncertainty distribution (including measurement and theory errors) is generally not known a priori, a simple, physically-reasonable form, such as a Gaussian or double-exponential distribution, is assumed, with statistical properties estimated from data residual analysis. In many cases, the full error covariance matrix (including off-diagonal terms) is required, and in some cases effects of nonstationary errors must be included. If biased data errors are suspected, additional unknown parameters representing the biases are included explicitly in the inversion. The validity/applicability of the above assumptions and estimates is examined a posteriori by applying both qualitative and quantitative statistical tests. New advances in efficient and adaptive MCMC sampling for nonlinear inversion will be presented.

\section{$8: 55$}

4aSP3. Particle filtering for dispersion curve estimation from spectrograms of acoustic signals. Ivan Zorych, Zoi-Heleni Michalopoulou (Dept. of Mathematical Sci., New Jersey Inst. of Technol., Newark, NJ 07102), James H. Miller, and Gopu R. Potty (Univ. of Rhode Island, Narragansett, RI 02882)

In this work we develop a particle filtering method for dispersion curve extraction from spectrograms of acoustic signals, the goal being to obtain accurate representation of modal dispersion for geoacoustic inversion. The approach combines particle filtering with modeling of sound propagation in ocean environments to track dispersion curves of multimodal signals in noisy environments. "Crispy" estimates of dispersion curves are extracted that are not characterized by extensive uncertainty typically hindering geoacoustic inversion. Results are presented from both synthetic signals and real data collected in the East China Sea during the ASIAEX experiment. [Work supported by ONR.]

\section{9:15}

4aSP4. Sampling with heuristics. Kevin H. Knuth (Dept. of Phys., Univ. at Albany, 1400 Washington Ave., Albany, NY 12222)

Algorithms that rely on heuristics are attractive to those interested in solving acoustic problems. The main reason is that heuristics often derive from solutions to a simplified problem and, as such, they are able to accurately estimate a subset of model parameters. However, algorithms that rely on heuristics are not sufficiently robust as they ignore vast regions of the parameter space. For this reason, sampling techniques that focus on exploration increase the probability of finding a difficult solution. However, sampling algorithms can become trapped in difficult problems that possess vast plateaus in the parameter space upon which the solution space occupies an extremely small volume. In these cases, heuristics at least have a chance to succeed where sampling is likely to fail. This work considers incorporating heuristically-obtained samples into a relatively new sampling algorithm called nested sampling. Nested sampling strives to localize the solution space while simultaneously integrating the posterior probability to compute the Bayesian evidence. The challenge is that nested sampling accomplishes this by working with uniformly distributed samples, whereas heuristics necessarily result in nonuniformly distributed samples. The difficulties and benefits in combining these two search strategies are described in the context of acoustic source separation.

9:35

4aSP5. The estimated ocean detector: Detection of signals with different parameter distributions. Jeffrey Ballard, Colin Jemmott, R. Lee Culver, and Leon Sibul (Grad. Prog. in Acoust., The Pennsylvania State Univ., P.O. Box 30, State College, PA 16804, jab795@psu.edu)

Earlier we presented a maximum likelihood receiver for acoustic signals that have propagated through a random or uncertain ocean, the estimated ocean detector (EOD) [Ballard et al., Oceans 2006, Boston, MA]. In general, the EOD incorporates statistical knowledge of signal uncertainty in the form of signal parameters probability density functions (pdfs). Note that Monte Carlo simulation and possibly other techniques can utilize deterministic and statistical knowledge of the environmental to predict signal parameter pdfs. The EOD utilizes the a priori signal parameter pdfs to estimate signal parameters from the data (or observations), then correlates the estimate with the data; hence, the name estimator-correlator is also used. Previously we showed that, for Gaussian signals embedded in Gaussian noise, the EOD reduces to the weighted sum of an energy detector and a correlation detector. This talk presents an EOD formulated to distinguish between signals whose parameters possess different a priori distributions. Performance is seen to depend upon the difference between parameter distributions for the two different signals. [Work supported by ONR Undersea Signal Processing Code 321US.]

9:55

4aSP6. Calibration of source level, expected transmitted power, and reverberation for long-range sonar in fluctuating range-dependent ocean waveguides. Purnima Ratilal, Mark Andrews, Ameya Galinde (Northeastern Univ., 409 Dana Res. Ctr., Boston, MA 02115), and Nicholas Makris (MIT, Cambridge, MA 02139)

The ocean waveguide is temporally and spatially varying. An acoustic signal transmitted through the ocean or scattered from objects will get randomized. Estimating parameters of sonar, the environment, and scatterers requires a statistical approach that incorporates medium uncertainties into the signal analysis. Here we discuss source level, transmission loss (TL), and reverberation model calibration using data acquired with a long-range sonar on the New Jersey STRATAFORM. Broadband acoustic data measured by a desensitized hydrophone in a towed horizontal receiving array at numerous locations from a vertical source array are analyzed. The match-filtered data are compared to the expected TL output from a parabolic equation model that accounts for bathymetric variations. A maximum likelihood estimator is implemented to provide a global inversion of the data for source level, attenuation due to scattering, and match-filter degradation in the multi-modal ocean waveguide. An estimate is also provided of the coherence bandwidth for broadband acoustic signal transmission in this environment. A challenge in calibrating bottom reverberation models with sonar data lies in separating the scattered intensity from moving objects, such as fish groups, and distinguishing them from the statistically stationary background reverberation. An approach is presented for this purpose. 
4aSP7. Application of multitaper methods to passive sonar. Kathleen Wage (George Mason Univ., 4400 Univ. Dr., MSN 1G5, Fairfax, VA 22030)

While the multitaper spectral estimation method [Thomson, Proc. IEEE 70, 1055-1096 (1982)] is used extensively in time series analysis, it has received much less attention in the array processing literature. The multitaper approach reduces the variance of power estimates by averaging windowed Fourier transforms of the data obtained using a set of orthogonal window functions. One advantage of the multitaper method is that it is designed to work with low sample support. Since multitaper estimates are often formed using a single data snapshot, they are suitable for use in highly nonstationary environments where it is difficult to obtain reliable estimates of the noise and interference statistics. This talk focuses on the application of the multitaper method to the passive sonar array processing problem. Standard techniques exist for detecting the presence of line components using multitaper estimates. This talk describes how to extend these techniques to detect plane waves in sonar data, where the signals are typically modeled as complex Gaussian rather than deterministic. Processing of non-plane-wave signals, e.g., normal modes, will also be briefly discussed. [Work supported by an ONR Young Investigator Award.]

\section{0:50}

4aSP8. Exploiting waveguide invariant reverberation striations for improved active sonar detection and classification. Ryan Goldhahn, Jeff Rogers, Granger Hickman, and Jeffrey Krolik (Duke Univ., Durham, NC 27708-0291)

Active sonar reverberation level (RL) uncertainty is exacerbated in shallow water by multipath propagation and multiple interactions with the bottom. This affects, for example, the statistics of split-window RL estimates used for cell-averaging constant false alarm rate (CA-CFAR) normalization of A-scan returns. For larger signal bandwidths, target detection is impacted by more incoherent reverberation components causing higher background variance while, alternatively, using smaller bandwidth signals results in higher range variability of the mean due to coherent multipath modal interference. In this paper, we present an alternative to CA-CFAR normalization that accounts for the frequency-selective fading characteristics of the multipath channel. The idea is to use the waveguide invariant property to estimate the frequency-dependent reverberation level at the range cell of interest using neighboring range cells at frequencies along striations in the time-frequency distribution of the sonar return. An approximate generalized likelihood ratio test (GLRT) detector is derived and demonstrated on acoustic tank data as well as Mediterranean active sonar data collected during the SCARAB-98 experiment [C. W. Holland, J. Acoust. Soc. Am. (2006)]. Detection and classification results indicate the considerable potential of a waveguide invariant-based approach. [Work supported by ONR321US.]

\section{1:10}

4aSP9. Sonar target tracking incorporating uncertain estimates of signal to noise ratio. Warren L. J. Fox, James W. Pitton, and Eric A. Rust (Appl. Phys. Lab., Univ. of Washington, 1013 NE 40th St., Seattle, WA 98105, warren@apl.washington.edu)

In the standard approach to sequential estimation for target tracking, tracks are formed on contacts that exceed some predefined detection threshold, set to achieve desired $P_{D}$ and $P_{F A}$ based on an expected SNR and a priori data probability PDFs. If the expected SNR is erroneous, degradation will be observed as either excessive false contacts or missed detections for weak targets. A method known as the Probabilistic Data Association Filter with Amplitude Information (PDAFAI) estimates SNR from the target and interference statistics, and uses the amplitude of the contacts together with the estimated SNR in the track association stage of the tracker. This method requires the PDF for the observed signal. In active sonar, this PDF is dependent on the expected SNR, which is a spatially and temporally varying quantity. Previous work has extended the PDAFAI method by adapting the tracker to the acoustic propagation environment via the measured contact amplitude together with the expected SNR derived from an acoustic propagation model, and by incorporating target strength estimates for contacts in developing tracks. Here, the method is extended further to include probabilistic descriptions of SNR, derived both from uncertainties in estimated environmental (e.g., bottom geoacoustic) and target properties.

\section{1:30}

4aSP10. Active target tracking using the bistatic invariance principle. Lisa Zurk and Chensong He (Portland State Univ., P.O. Box 751, Portland, OR 97207, zurk1@ cecs.pdx.edu)

The acoustic pressure received from a moving source in a shallow water channel is highly variable and depends critically on characteristics of the channel such as bathymetry, sound speed, and bottom properties. Past work has shown that knowledge of the channel properties can be embedded in physics-based signal processing algorithms for increased system performance. However, this requires accurate knowledge of the channel characteristics, which are often poorly known. An alternate approach is to identify aspects of the field structure that are invariant to small perturbations in the propagation environment. The waveguide invariant is a scalar parameter that has been used extensively in passive sonar to interpret features such as intensity maxima in lofargrams. Recent work has used experimental data to demonstrate that a similar parameter exists for active geometries, although the invariant relationship is more complicated because of the multiple propagation paths. In this presentation active invariant striations are exploited in an active tracker formulation. The tracker utilizes a state space representation that includes the time-dependent frequency content of the pulse as one of the sonar observables. In addition to target kinetics, the time-frequency structure provides track constraints that improve discrimination of target versus bottom reverberation. [Work sponsored by ONR-321US.] 


\title{
Session 4pAAa
}

\section{Architectural Acoustics: Workshop for American Institute of Architects Continuing Education Units Presentation}

\author{
Bennett M. Brooks, Chair \\ Brooks Acoustics Corporation, 27 Hartford Turnpike, Vernon, CT 06066
}

Invited Papers

1:30

4pAAa1. Introduction to workshop goals. Anthony K. Hoover (Cavanaugh Tocci Assoc., 327 F Boston Post Rd., Sudbury, MA 01776) and Bennett M. Brooks (Brooks Acoust. Corp., Vernon, CT 06066)

Many states require that architects obtain continuing education unit (CEU) credits annually, in order to maintain their registration or licensure. The American Institute of Architects (AIA) Continuing Education System (CES) offers continuing education courses, which may be given by third party providers. The Technical Committee on Architectural Acoustics (TCAA) is an AIA/CES Registered Provider. The goal of this workshop is to prepare members of the TCAA so that they may be authorized to present a short course that can earn attendees CEU credit. In order for TCAA members to qualify to meet the AIA requirements, they must attend this workshop, which is given in two parts. The first part will be devoted to the short course presentation material, which is in a standardized format. The second part of the workshop will focus on the AIA CEU Program registration and reporting requirements. Of course, anyone is free to register with the AIA to provide their own CEU program. However, the advantages of participating in this program are that the TCAA short course is already prepared, is preapproved by the AIA, and the registration fees are paid by the Acoustical Society of America.

$1: 45$

4pAAa2. Technical Committee on Architectural Acoustics short course presentation material. Anthony K. Hoover (Cavanaugh Tocci Assoc., 327 F Boston Post Rd., Sudbury, MA 01776)

The Technical Committee on Architectural Acoustics (TCAA) is a Registered Provider in the American Institute of Architects (AIA) Continuing Education System (CES). The TCAA has developed a standardized introductory short course for architects, called Architectural Acoustics. An architect can earn one continuing education unit by attending this short course, if it is presented by a qualified member of TCAA. The course covers topics in sound isolation, mechanical system noise control, and finish treatments. This paper will cover the course material in order to prepare and qualify potential presenters. There will be time at the end of the paper for questions and answers on the course material. In order to qualify as an authorized presenter for this AIA/CES short course, attendance at this workshop and membership in TCAA are required.

$$
\text { 2:45-3:00 Break }
$$

\section{3:00}

4pAAa3. American Institute of Architects/Continuing Education System (AIA/CES) provider registration and reporting requirements. Bennett M. Brooks (Brooks Acoust. Corp., 27 Hartford Tpke., Vernon, CT 06066)

The Technical Committee on Architectural Acoustics (TCAA) is a Registered Provider in the American Institute of Architects (AIA) Continuing Education System (CES). The TCAA has developed a standardized introductory short course for architects. The TCAA short course is called Architectural Acoustics and attendance at this one-hour long course can earn an architect one continuing education unit (CEU). This paper will cover the administrative requirements of the AIA/CES, to prepare potential presenters. These requirements include the proper handling of paperwork, so that AIA members may receive credit for the course. Also, the manner in which the course is given is dictated by AIA requirements. TCAA membership and attendance at this workshop are required to qualify as an authorized presenter for this AIA/CES short course. 


\title{
Session 4pAAb
}

\section{Architectural Acoustics: Predictions and Simulation}

\author{
Paul T. Calamia, Chair \\ Rensselaer Polytechnic Inst., School of Architecture, 110 8th St., Troy, NY 12180 \\ Chair's Introduction-3:45
}

Contributed Papers

3:50

4pAAb1. Modifications to the diffusion model for room-acoustic prediction. Yun Jing and Ning Xiang (School of Architecture, Rensselaer Polytechnic Inst., 110 8th St., Troy, NY 12180-3590)

Recently, a diffusion model has drawn attention in room-acoustic predictions. This paper proposes possible modifications to the diffusion model to predict the reverberation times and sound pressure distributions in enclosures. While the original diffusion model [Ollendorff, Acustica 21, 236-245 (1969); J. Piacut et al., Acustica 83, 614-621 (1997); Valeau et al., JASA 119, 1504-1513 (2006)] usually has a good performance for low absorption surfaces, the modified diffusion model, including a new boundary condition and a higher order approximation of a transport equation, yields more satisfactory results for both low and high absorption surfaces. Examples of cubic rooms, flat rooms, and long rooms with various absorption coefficients are presented for comparisons between the modified model, the original model, a geometrical-acoustics model, and several well-established theories in terms of reverberation times and sound pressure level distributions. This paper will discuss improved prediction accuracies by the modified diffusion model.

\section{4:05}

4pAAb2. Investigation of voice stage support: A subjective preference test using an auralization system of self-voice. Cheuk Wa Yuen and Paul T. Calamia (Grad. Program in Architectural Acoust., School of Architecture, Rensselaer Polytechnic Inst., 110 8th St., Troy, NY 12180, wa@amusical.org)

The human voice plays an integral role in dramatic art. The performance of singers and actors, who perceive their voice through their ears as well as bone conduction, is highly related to the acoustic condition they are in. Due to the proximity of the sound source and the spectral difference in the transmission through the skull as compared to air, a support condition different from that for musical instrumentalists is needed. This paper aims at initiating a standardization of methodology in subjective preference testing for voice stage support in order to collect more data for statistical analysis. A proposal of an acquisition/auralization system for self-voice and a set of subjective test procedures are presented. The subjective evaluation of the system is compared to previous designs reported in the literature, and the implementation is validated. A small playhouse has been measured and auralized using the system described, and subjective-preference tests have been conducted with 13 professionally trained actors. Their preferred stage-acoustic conditions (in relation to locations on stage and head orientations) are reported. The results show potential directions for further investigations and identify the necessary concerns in developing an objective parameter for voice stage support.
$4: 20$

4pAAb3. Validation study of computer modeling of source directivity. Michelle C. Vigeant and Lily M. Wang (Architectural Eng. Prog., Univ. of Nebraska-Lincoln, Peter Kiewit Inst., 1110 S. 67th St., Omaha, NE 68182-0681,mvigeant@mail.unomaha.edu)

Previous research has focused on the changes in objective and subjective results when changing source directivity in a computer model, but little work has been done to validate these simulated changes experimentally. An investigation was carried out to compare the measured and simulated room acoustics parameters which result from using a directional sound source, a JBL Eon-10 G2 loudspeaker. Measurements were made in a 100-seat lecture-style classroom, using the sine sweep method, with (a) an omni-directional and (b) the directional loudspeaker oriented in four directions. The measured differences in reverberation time (T30) were minimal across the two source types and four orientations, while significant differences resulted for early decay time (EDT) and clarity index (C80). An ODEON v6.5 model of the classroom was calibrated against the omni-directional results to within two just-noticeable-differences (JNDs) across the three parameters of interest: T30, EDT, and C80. Simulations with the directivity of the JBL loudspeaker were then performed and the results differed by less than two JNDs from the measurements for all source-orientation/receiver combinations. In conclusion, ODEON v6.5 does accurately model the changes in room acoustic parameters which result from using different source directivities. [Work supported by the National Science Foundation.]

$4: 35$

4pAAb4. Modeling room impulse response by incorporating speaker polar response into image source method. Zhixin Chen and Robert Maher (Dept. of Elec. and Computer Eng., Montana State Univ., Bozeman, MT 59717-3780)

Simple computer modeling of impulse responses for small rectangular rooms is typically based on the image source method, which results in an impulse response with very high time resolution. The image source method is easy to implement, but the simulated impulse responses are often a poor match to measured impulse responses because the description of the source is often too idealized to match the real measurement conditions. For example, the basic image source method has often assumed the sound source to be an omni-directional point source for ease of implementation, but a real loudspeaker may include multiple drivers and exhibit an irregular polar response in both the horizontal and vertical directions. In this paper, an improved room impulse response computer modeling technique is developed by incorporating the measured horizontal and vertical polar responses of the speaker into the basic image source method. Results show that compared with the basic image source method, the modeled room impulse response using this method is a better match to the measured room impulse response. 
4pAAb5. Increasing the direct-to-reverberant ratio in rooms with a constant-beamwidth, wide-bandwidth loudspeaker array. Douglas F. Winker (Acoust. Systems-A Div. of ETS-Lindgren, 415 E. St. Elmo Rd., Austin, TX 78745) and Elmer L. Hixson (Univ. of Texas, Austin, TX 78712)

A constant-beamwidth, wide-bandwidth (CBWB) loudspeaker array was used to improve speech intelligibility in two rooms. The CBWB loudspeaker array maintained a constant beamwidth between $500 \mathrm{~Hz}$ to $4 \mathrm{kHz}$. The array had a narrow vertical beam pattern and a wide horizontal beam pattern. During the study, an increase in the direct-to-reverberant ratio was observed that correlated with an increase in speech intelligibility (measured with the STI method). The correlation between improved speech intelligibly and an increased direct-to-reverberant ratio will be discussed, and the relation between each source's beam pattern and the direct-toreverberant ratio will be presented. The array was compared to a typical PA source and showed increases in the direct-to-reverberant ratio. Room simulations were conducted with CATT-Acoustic and compared to realworld measurements. The study showed an increase in the direct-toreverberant ratio in each room, which corresponded to improved speech intelligibility.
4pAAb6. Qualitative analysis of concert hall acoustics with multiple directional sources. Youngmin Kwon, Gary W. Siebein, and Martin A. Gold (Architecture Technol. Res. Ctr., Univ. of Florida, 134 ARCH, P.O. Box 115702, Gainesville, FL 32611, ykwon@acousticdimensions.com)

Following the previous work on quantitative analysis of concert hall acoustics with multiple directional sources (16 directional loudspeakers) [Kwon and Siebein, J. Acoust. Soc. Am. 120, 3263(A) (2006)], this paper discusses qualitative assessments of concert hall acoustics by means of psycho-acoustical listening tests of room acoustical qualities such as loudness, reverberance, clarity, warmth, spaciousness, envelopment, etc. The music signals binaurally recorded in a performance hall with the same multiple directional source array were evaluated in comparison to those recorded with the single omni-directional source. Results showed that some of the room acoustical qualities of the music signals recorded with multiple directional sources were perceived noticeably different from those recorded with a single omni-directional source. In the main orchestra area at $95 \%$ significance level, the music recording with multiple directional sources was perceived more in reverberance and clarity but less in warmth. Some results from these qualitative assessments were, however, found to contradict their quantitative measures.

THURSDAY AFTERNOON, 7 JUNE 2007

SALON A, 1:30 TO 3:20 P.M.

\title{
Session 4pEA
}

\section{Engineering Acoustics: Acoustical Measurements and Methods II}

\author{
Daniel M. Warren, Chair \\ Knowles Electronics, 1151 Maplewood Dr., Itasca, IL 60143
}

Chair's Introduction-1:30

Contributed Papers

$1: 35$

4pEA1. $60 \mathrm{MHz}$ multidomain ultrasonic transducer. Igor $\mathrm{V}$. Ostrovskii and Andriy B. Nadtochiy (Dept. of Phys., Univ. of Mississippi, University, MS 38677)

Multidomain periodically poled structure of 80 inversely poled domains of 50-micron-width each was fabricated in a 350-micron-thick 42degrees-Y-rotated cut of $\mathrm{LiTaO} 3$ wafer. A multidomain structure in a ferroelectric plate is characterized by its own domain resonance where acoustic wavelength is equal to the domain period, and ultrasound speed is $\sim 6$ km/s for LiTaO3 [I. V. Ostrovskii and A. B. Nadtochiy, Appl. Phys. Lett. 86, 222902 (2005)]. The rf impedance of the transducer as a function of frequency demonstrates a sharp resonance-antiresonance behavior at 57.6-60 MHz band (sample LTYR42B5a). The resonance observed is a pure multidomain resonance because crystal length is much longer than domain width. Overall dimensions of this transducer are $4 \times 4 \times 0.35 \mathrm{~mm}$ ${ }^{3}$, and irradiating surface area is $4 \times 0.35 \mathrm{~mm}^{2}$. Two such transducers were used to model a delay line with water as a medium of propagation. A 1- $\mu \mathrm{s}$ burst with $59 \mathrm{MHz}$ central frequency was delayed for $4.3 \mu \mathrm{s}$ in the water. In conclusion, we present a new high frequency transducer for various applications in non-destructive testing, medicine, and biology [Patent Pending]. Experimental measurements and theoretical calculations are in good agreement.
$1: 50$

4pEA2. Head and torso simulators. Gunnar Rasmussen (Skovlytoften 33, 2840 Holte, Denmark, gr@gras.dk)

The KEMAR was developed by Knowles Electronics and introduced in 1972, thoroughly described by Mahlon D. Burkhard, and standardized in ANSI S3.361ASA58-1985. The KEMAR was provided with ears typical of American and European females as well as Japanese males and females. Larger ears more typical of American and European males have been developed with well-defined hardness of 0055 and 0035 shore. The ears are readily exchanged with the ITU P57 Type 3.3 ear. The KEMAR is designed to offer lifelike reproducible test conditions and is therefore based on average anthropometric dimensions of an adult human, while other HATS are designed using simplified or average geometric convenient dimensions. The KEMAR may be provided with an artificial mouth system with well-defined characteristics. The KEMAR also offers welldefined positioning of microphones for linearization as well as positioning systems for handheld communication systems. Measurements show good agreement with early data published by M. D. Burkhard and R. M. Sachs [“Anthropometric Manikin for Acoustic Research," JASA 58, 214-222 (1975)]. Conclusion: Acoustical measurements carried out since 1972 can still be compared and carried out on a system, which has not changed acoustically except for increased versatility and improved acoustic documentation. 
upstream acoustic modes and downstream convective modes, coupled together by boundary conditions, and driven by mass addition and removal

4pEA3. An etude in hearing-aid directivity measurement and benchmarking. Matthew J. Green and Thomas Burns (Starkey Labs, 6600 Washington Ave. S., Eden Prairie, MN 55344)

In this study, a three-dimensional, automated scanning system is used to measure the directional performance of hearing aids mounted on a test manikin. A diffuse field is simulated in an anechoic environment by sequentially positioning a loudspeaker along the surface of a 1-m-radius sphere circumscribing the manikin and device under test. Particular attention is given to the type of stimulus, the spatial resolution of loudspeaker positions, and postprocessing of the impulse responses; each affects the measurement precision of the Directivity Index and the 3D polar responses. The purpose of this research is to describe the recipe that yielded minimal measurement bias and variance for ITEs, BTEs, and OTEs of varying directivity.

\section{2:20}

4pEA4. Methodology of planar unsteady flow measurements in an excised larynx. Shanmugam Murugappan, Sid Khosla, Ephraim Gutmark, and Raghava LakhamRaju (Dept. of Otolaryngol.-HNS, Univ. of Cincinnati, 231 Albert Sabin Way, \#MSB 6308, Cincinnati, OH 45267)

A methodology to obtain phase locked planar unsteady flow of the near field of supraglottal flow in an excised larynx using particle image velocimetry (PIV) is presented. Different steps involved in obtaining 2D flow field at different instances of the EGG or microphone signal and the structural motion of the vocal fold are discussed. Real time signal processing of the sensor signal coupled with the synchronization of the PIV flow field and high speed imaging of the vocal fold is used to evaluate the relationship between acoustics-structure and flow. Issues that can affect the accuracy of the flow field measurements and the reconstruction of the flow patterns at different instances during the EGG/microphone cycle were identified and suitable measures taken to resolve them are discussed. Some of these include simulating a symmetric vocal fold motion, maintaining a constant phonation frequency over the entire experimental run, preventing any spurious vectors that could arise due to reflection from the moist tissue surface or poor spatial or temporal resolution. Examples are also shown where the effect of these factors impact both the raw seeded image and correlation PIV algorithm used to extract the flow field vectors.

\section{2:35}

4pEA5. Flow induced aeroacoustic oscillations in a tube with a region of abrupt cross-sectional area increase. Donald Bliss and Noah Sakimura (Mech. Eng., Duke Univ., Durham, NC 27708)

The phenomenon of aeroacoustic oscillations induced by flow through a circular tube with an annular cavity region formed by an abrupt area increase is studied. At the beginning of the area increase, the flow separates and produces a shear layer that subsequently impinges on the downstream end of this region. The shear layer is a medium for propagation of convective vorticity waves. In the surrounding cavity, axial acoustic wave propagation can occur. The central flow region is governed by the convective wave equation, and the simple wave equation applies in the cavity. Coupled axial modes are identifiable as being primarily convective or primarily acoustic. The oscillation mechanism involves the interaction of at the rear of the annular cavity caused by shear layer impingement on downstream edge. The oscillation process is analyzed using a crosssectional averaging method for quasi one-dimensional waves to determine system wavenumbers and eigenmodes. Oscillation frequencies are determined by the condition of maximum energy extraction from the mean flow, arising from unstable convective waves, and by a compatible shear layer phase condition. A comparison with simple experiments is presented.

$$
\text { 2:50 }
$$

4pEA6. Chaotic state in an electrodynamic loudspeaker controlled by gas pressure. Ivan Djurek, Antonio Petosic (Faculty of Elec. Eng. and Computing, Dept. of Electroacoustics, Unska 3, HR-10000, Zagreb, Croatia), and Danijel Djurek (AVAC, Kesten brijeg 5, Remete, HR-10000 Zagreb, Croatia)

An electrodynamic loudspeaker with resonant frequency $f_{0}=47.1 \mathrm{~Hz}$ has been driven in the far nonlinear regime, and $f_{0}$ increases with increasing driving ac current $I_{0}$. Landau cutoff of the vibration amplitude appears at frequency $f_{c}>f_{0}$, which is followed by the doubling of driving period $1 / f$ and appearance of harmonic sequences at $\frac{1}{2} n f, \frac{1}{4} n f, \frac{3}{4} n f, \ldots$. By further increase of current the white noise spectrum appears, which is characteristic of the chaotic state. Electrodynamic loudspeaker is represented by an ordinary differential equation of motion describing an anharmonic forced oscillator, and it is possible to achieve an independent control of chaotic state by the gas pressure, since real $R_{S}$ and imaginary part $X_{S}$ of the gas acoustic impedance affect, respectively, friction and inertial terms in the equation. The used gas atmospheres $(0.01<p<1$ bar $)$ were $\mathrm{H}$ ${ }_{2}, \mathrm{D}_{2}, \mathrm{He}_{4}, \mathrm{Ne}, \mathrm{Ar}, \mathrm{CO}_{2}, \mathrm{SF}_{6}$, and air. The cutoff frequency $f_{c}$ depends on the gas pressure and it was plotted against $\left(p, I_{0}\right)$, which in turn defines the surface in three-dimensional diagram. Values of parameters $\left(p, I_{0}\right)$ triggering chaotic state were chosen above this surface, and universality of some laws describing such state was tested against the gas density.

\section{3:05}

4pEA7. Ultrasonic resonance spectroscopy for cryogenic materials. Petre Petculescu, Remus Zagan (Ovidius Univ., 124 Mamaia Blvd., Constanta 900572, Romania), and Andi Petculescu (Univ. of Louisiana-Lafayette, Lafayette, LA 70504)

Cryogenic steels are needed in the construction of industrial installations able to withstand low temperatures. This poster presents results on the use of an ultrasonic resonant technique to determine the composition of the cryogenic stainless steel 10TiNiCr180 with an austenitic structure. The investigations were done both in the base material and in the weld, with and without thermal treatment. Upon exposing the sample to two thermal treatments (welding-hardening and welding-hardening-annealing), metallographic analysis showed the presence of chromium carbide in the base material and the weld. The ultrasonic power spectrum shows a prevalent austenitic structure in the base material and a compound austeniteferrite-chromium carbide structure in the weld. The grain size determined from the spectral peak ratio is compared with the one obtained by metallographic analysis. The measurements presented here were obtained in direct contact with the pulse-echo technique, with excitation centered at 5 $\mathrm{MHz}$. 


\title{
Session 4pMU
}

\section{Musical Acoustics: "Myths and Mysteries" in Musical Acoustics}

\author{
Evan B. Davis, Cochair \\ Boeing Commercial Airplanes, 8556 Burke Ave., N., Seattle, WA 98103 \\ Thomas R. Moore, Cochair \\ Rollins College, Dept. of Physics, Winter Park, FL 32789
}

Chair's Introduction-2:00

Invited Papers

2:05

4pMU1. Myth-busting brass instrument design. Richard A. Smith (Smith-Watkins Brass, Richard Smith (M I) Ltd., Cornbrough, Sheriff Hutton, York, YO60 6RU, UK)

Richard Smith has designed and made instruments for professional brass players for over 35 years. With a background as scientist and musician, he has a unique view of the subject in which his myth-busting has been a dominant feature. The challenge now is to transfer this knowledge to music educators and players. It can be shown that any instrument is a compromise of musical qualities, making the quest for the perfect instrument pointless and a distraction from the real business of making music. Resistance is probably the most difficult of these musical qualities to interpret from player responses. It will be demonstrated how the language of fluid dynamics has been wrongly adopted by players, educators, and manufacturers to give pseudo-scientific authority to their teaching and product promotion. The development of a blindfold testing technique by Smith was crucial to (a) the study of materials used in instrument construction and (b) a breakthrough in efficient instrument design. Current work with Thomas Moore (Rollins College, USA) and David Howard (University of York, UK) investigates the mystery of hi-note playing on the trumpet where the most skilled players find distinct slots above $2 \mathrm{kHz}$ in, according to acoustical textbooks, a resonance free zone.

\section{$2: 25$}

4pMU2. It's all in the bore! ${ }^{\text {a) }}$-Is that true? Aren't there other influences on wind instrument sound and response? Wilfried Kausel (Inst. of Musical Acoust., Univ. of Music and Performing Arts, Anton von Webernplatz 1, A-1030 Vienna, Austria, kausel@mdw.ac.at)

A review of the current state of knowledge concerning the influence of wall vibrations on the sound of brass wind instruments, flue pipes, and woodwind instruments is given. The question of whether this influence is strong enough to be objectively observable is still a controversial issue. While instrument makers and musicians make a strong claim that wall thickness, material, and conditioning are crucial factors for sound quality and response of wind instruments, acousticians tended —at least in the past—-to attribute all acoustical characteristics to the actual bore profile. Recently this seems to be changing. First, there is growing experimental evidence favoring the hypothesis that wall vibrations do matter; second, theoretical models are starting to be developed which deal with the interaction between the air column sound field and its oscillating boundary conditions. Another suggested theory is that wall vibrations are propagated back to the mouthpiece interacting with the player's lips affecting the delicate and strongly nonlinear mechanism of sound generation. Even the question of whether vibrating bells can radiate a significant amount of sound does not seem to have reached a final answer. New experiments have been conducted to help answer these open questions. ${ }^{\text {a) }}$ R. A. Smith, "It's all in the bore!," International Trumpet Guild Journal, 12(4) (1988).

4pMU3. Vibroacoustics of musical wind instrument: Analysis of some pathological and nonpathological behaviors. Francois Gautier, Guillaume Nief, Joel Gilbert, and Jean-Pierre Dalmont (Laboratoire d'Acoustique de l'Universite du Maine, UMR CNRS 6613, Av. O. Messiaen, 72085 Le Mans cedex 9, France)

Wall vibration of a musical wind instrument can be clearly noticed and measured in playing configuration. However, the problem of quantifying its effect on the emitted sound remains a subject of debate. Wall vibrations are generated by two mechanisms: (1) a mechanical source corresponding to the impacts of a reed (or musicians lips) on the mouthpiece and (2) an acoustical source due to the internal sound field. In this paper, we present an investigation of mechanism (2) using an experimental approach and a theoretical model of a generic simplified instrument (cylindrical vibrating shell, with a slightly distorted circular cross section). Analysis leads to the conclusion that, overall, vibroacoustic couplings are very small and do not induce any audible contribution. However, the wall vibration can play a significant role for some particular choices of material and geometry, which lead to coincidences between structural and acoustical modes. In these configurations, model and experiments show that the input acoustic impedance is clearly perturbed by wall vibrations. Using a blowing machine, it is shown that these perturbations can induce some timbre changes or some unstable oscillations regimes. Analysis of such pathological behaviors will be presented. 
4pMU4. On the need for musical instrument strings to dynamically stretch. Evan B. Davis (Boeing Commercial Airplanes, 8556 Burke Ave N., Seattle WA 98103)

Given clamped or fixed boundary conditions a string must stretch or elongate to vibrate. Many have analyzed the effect of string stretch under the fixed boundary condition assumption and the related nonlinear motion of the string. However, if the boundary of the string at the soundboard was fixed the soundboard would not move. A rigid soundboard is not a very useful design for the production of music. It will be shown that there are geometries which allow soundboard motion without string stretch well as geometries that require string stretch for soundboard motion.

\title{
Contributed Papers
}

$3: 25$

4pMU5. To play it is to improve it: An analysis of the "playing-in" phenomenon of stringed instruments. Andrew A. Piacsek (Dept. of Phys., Central Washington Univ., Ellensburg, WA 98926-7422)

There is a growing interest in resolving the question of whether, and how, the act of playing a stringed instrument (in the violin and guitar families) improves its sound and playability over time. Although generally accepted by musicians, the causal connection is difficult to test due to the very large number of potentially relevant parameters, both physical and psychological. This presentation describes various historical references, interviews with musicians, and recent research on the subject of the "playing-in" phenomenon. Results will be presented from new experiments on the response of spruce panels subject to mechanical stimulus.
$3: 40$

4pMU6. How can bell vibrations affect the sound of brass instruments? Thomas Moore, Vishal Jiawon, and Daniel Zietlow (Rollins College, Winter Park, FL 32789, tmoore@ rollins.edu)

It has recently been shown that the vibrations of the bell of a trumpet affect the spectra of the sound produced during play. The manner in which these vibrations produce this effect is still under investigation, but two possibilities have been proposed: (1) the vibrations of the bell feedback to the lips and change their motion, and (2) the bell vibrations change the radiation impedance of the instrument. The former explanation appears to be the most plausible; however, we will present results of recent experiments that indicate at least some of the effect is due to a change in radiation impedance.

THURSDAY AFTERNOON, 7 JUNE 2007

CANYON ROOM A, 1:50 TO 5:00 P.M.

\section{Session 4pNS}

\section{Noise, Engineering Acoustics, and Signal Processing in Acoustics: Recent Advances in Active Noise Control II}

\author{
Scott D. Sommerfeldt, Cochair \\ Brigham Young Univ., Dept. of Physics and Astronomy, Provo, UT 84602-4673 \\ Kenneth A. Cunefare, Cochair \\ Georgia Inst. of Technology, School of Mechanical Engineering, 113 MRDC II, Love Building Atlanta, GA 30332-0405 \\ Invited Papers
}

$1: 50$

4pNS1. Advanced digitally adjustable analog feedback control system and its usage in active noise insulation. Marko Antila, Hannu Nykanen, Jari Kataja, and Velipekka Mellin (VTT Tech. Res. Ctr. of Finland, POB 1300, FIN-33101 Tampere, Finland)

Feedback control for the active noise cancellation can be done with analog or digital circuitry. Although digital approach is steadily gaining ground, analog control still has its uses. Short control latency is the feature of an analog controller-it has the shortest possible delay. Its drawback is the limited flexibility for adjustable or adaptive solutions. To overcome this, digitally adjustable analog controllers can be used. A potential implementation for such controller uses field programmable analog array (FPAA) technology. With FPAAs both the filter parameters and structure are digitally adjustable, even during the operation. A development environment around the FPAA controller has been devised. It consists of the measurement system, an optimizer that calculates the optimum controller response, programming software, and actual FPAA hardware with its embedded software. The complete system is packaged tightly to a compact form. It can be used for evaluation purposes in acoustics laboratory conditions. Such a system has been evaluated in active noise insulation application. It has proven to operate as simulated and optimized also in real-world tests. The future development of the system includes further miniaturization, controller networking, and possible integration inside the sound insulation system. 
4pNS2. Rapidly converging adaptive state-space-based multichannel active noise control algorithm for reduction of broadband noise. Arthur P. Berkhoff (TNO Sci. and Industry, P.O. Box 155, 2600AD Delft, The Netherlands, arthur.berkhoff@tno.nl) and Johan M. Wesselink (Univ. of Twente, 7500AE Enschede, The Netherlands)

Rapidly changing spectra may lead to performance limitations in adaptive systems for broadband active noise control, especially in multichannel systems. This paper presents techniques to address the negative consequences of two main causes. First, the dynamics of the transfer paths between the noise control sources and the error microphones is compensated for by using a regularized state-space based adaptive filtered-error scheme. Second, for the reference signals a modified adaptive scheme is used, taking into account the nonwhiteness of these signals as well as the correlation between the individual signals. Examples are given for simulated data and for real-time implementations. [This work was partly supported by EC Contract 501084 (InMAR).]

2:30

4pNS3. Optimization of control source locations in an active noise control application of axial cooling fans using genetic algorithms. Connor R. Duke, Scott D. Sommerfeldt, and Kent L. Gee (Brigham Young Univ., N-281A ESE Provo, UT 84602, crd26@byu.edu)

By placing control sources in the near field, global attenuation of an axial cooling fan's blade passage frequency and harmonics can be achieved using active noise control. The optimal position of control sources when modeling a fan, whose characteristics differ significantly from a single monopole source or when modeling more than one fan, will be discussed. The number of control sources, as well as position of control sources, are optimized for specific primary source configurations using genetic algorithms. Source strength of control sources are calculated analytically to minimize radiated sound power. The results of optimization using different forms of genetic algorithms will be presented and compared. The results obtained from the genetic algorithms will be compared to experimental results using multiple sources.

4pNS4. Noise annoyance reduction using active control. Gerard Mangiante and Georges Canevet (Laboratoire de Mecanique et d'Acoustique, CNRS, 31 Chemin Joseph Aiguier, 13402 Marseille Cedex 20, France, mangiante@1ma.cnrs-mrs.fr)

In two former studies [G. Canevet and G. Mangiante "Psychoacoustic Assessment of Active Noise Control," Active 2004 (2004), and G. Mangiante and G. Canevet “Active Reduction of Environmental Noise: A Sound Quality Evaluation,” Internoise 2006 (2006)], the psychoacoustical effects of active noise control were described. One of these effects is the reduction of noise annoyance. This contribution aims at describing this annoyance reduction using the following indicators: loudness, sharpness, unbiased annoyance, and acoustic comfort. These indicators were calculated with a psychoacoustic-analysis software in which the following models were implemented: Moore and co-workers (1997), Zwicker and Fastl (1999), and Glasberg and Moore (2002). Using this software, various noises, selected from our everyday environment, were tested to investigate the effects of the following parameters: the width and the center frequency of the controlled band, the maximum attenuation in this band, and the sound pressure level of the noise before active control. The results showed that, in all cases, acoustic comfort is positively affected by active control. The annoyance reduction is greatly dependent of the shape of the power spectrum of the noise to be reduced. The best results are obtained for broadband noises with an energy contribution concentrated at low frequencies.

\section{3:10}

4pNS5. Eigenvalue equalization applied to the active minimization of energy density in a mock helicopter cabin. Stephan $\mathrm{P}$. Lovstedt (Dept. of Phys. and Astron., Brigham Young Univ., N283 ESC Provo, UT 84602, s_lovstedt@yahoo.com), Jared Thomas, Scott D. Sommerfeldt, and Jonathan Blotter (Brigham Young Univ., Provo, UT 84602)

A number of applications in active noise control require the ability to control and track multiple frequencies. If a standard filtered-x algorithm is used, the system must be designed to be stable for the slowest converging frequency anticipated, thereby leading to reduced overall performance of the system. Previous work has focused on overcoming this through development of a method that equalizes the eigenvalues of the system over the operating frequency range, leading to more uniform performance. The current work has built on the previous work to extend the method for implementation in systems that control the acoustic energy density. Minimizing energy density has been shown to have favorable performance characteristics when used for controlling enclosed acoustic fields. Thus, combining the approach of equalizing the system eigenvalues with energy density control leads to a system that incorporates the advantages of both methods. The control approach is demonstrated through implementation in a mock helicopter cabin, to demonstrate the favorable convergence characteristics, along with the global control of the field.

\section{3:30-3:45 Break}




\section{Contributed Papers}

4pNS6. Active noise reduction communication earplug for helicopter crew. Jason A. Solbeck, Matthew Maher, Toby Deitrich (Sound Innovations, Inc., 55 Railroad Row, White River Junction, VT 05001), Laura R. Ray, and Robert D. Collier (Dartmouth College, Hanover, NH 03766)

A custom designed shallow insert communication earplug for helicopter crews consists of a system based on a patented hybrid ANR feedbackfeedforward algorithm [Ray et al., J. Acoust. Soc. Am. 120(4), 20262038 (2006)]. The hybrid system provides the benefits of feedback ANR while extending the bandwidth and magnitude of total ANR performance with feedforward ANR, which improves attenuation of tonals. The development includes optimized algorithms, a custom earplug, and a miniaturized battery powered ANR module, weighing approximately $250 \mathrm{~g}$. The hybrid ANR earplug requires two miniature microphones, one inside and one external to the earplug and an internal speaker to deliver the cancellation signal. The software automatically adapts the ANR algorithm to the transfer function characteristics of the system to both improve performance and accommodate individual human fitting differences. The testing protocol and results are based primarily on measurements with a HEAD Acoustics manikin in the Sound Innovations sound room [Duncan et al., Proceedings of Internoise 2006, December 2006]. MIRE testing is planned for the Spring of 2007. Results to-date demonstrate comparable passive attenuation to a commercially available communication earplug and an added average active attenuation of approximately $6 \mathrm{~dB}$ for UH-60 helicopter noise. [Work supported by U.S. Army.]

\section{4:00}

4pNS7. NASA-sponsored active fan noise control research. Joe Posey and Michael Jones (NASA Langley Res. Ctr., Hampton, VA 23681, joe.w.posey@nasa.gov)

The National Aeronautics and Space Administration (NASA) has espoused the vision of developing technology capable of keeping all objectionable aircraft noise within airport boundaries. In order to achieve such an aggressive goal, new aircraft will have to be designed employing quiet propulsion, quiet lift, and quiet drag. Research activities are continuing on all these fronts. Dominant sources of propulsion noise to date on jetpowered transports have been jet noise and fan noise. As bypass ratios of aircraft turbofan engines have increased to improve fuel efficiency, jet velocities (and therefore jet noise) have decreased, making fan noise the larger contributor in many instances. In NASAs Advanced Subsonic Technology (AST) Program, which ran from 1994 until 2001, several active control strategies for tonal fan noise were investigated. These included rings of wall-mounted actuators, active impedance control, stator-mounted actuators, and hybrid active/passive concepts. The most promising approach seems to be stator-mounted actuators to provide control sources effectively co-located with the rotor/stator interaction source. The major remaining issue for active control of fan tones is the availability of highpowered, compact, efficient actuators for stator mounting. Control of broadband fan noise has been addressed, but much work remains to be done.

\section{4:15}

4pNS8. Determination of optimal near-field error sensor locations for active control of cooling fan noise using spherical harmonic expansions. Benjamin M. Shafer, Kent L. Gee, Scott D. Sommerfeldt, and J. Isaac Fjeldsted (Dept. of Phys. and Astron., N283 ESC, Provo, UT 84602, otishobbes@gmail.com)

Recent developments in the active control of cooling fan noise have used near-field error sensors to drive the pressure to zero [K. L. Gee and S. D. Sommerfeldt, J. Acoust. Soc. Am. 115, 228-236 (2004)]. Theoretical mapping of near-field pressure during minimization of sound power re- veals the location of pressure nulls that can be used to optimize the location of the error sensors. To this point, the locations of error sensors have been determined by modeling both the fan and the control loudspeakers as point monopoles. However, noise from an axial fan has monopole as well as multipole characteristics. The multipole characteristics of the fan can be obtained using a procedure based on the work of Martin and Roure [T. Martin and A. Roure, J. Sound Vib. 201, 577-593 (1997)]. Pressure values are obtained over a hemisphere in the far field of a primary source and the contributions from multipoles up to the second order, centered at the primary source, may be calculated using spherical harmonic expansions. The source information is then used in the aforementioned theoretical nearfield calculation of pressure. Error sensors are positioned using this calculation and the global attenuation is compared to that of previous experiments.

\section{$4: 30$}

4pNS9. Near field placement of error sensors in an active noise control application of axial cooling fans using flow visualization techniques. Connor R. Duke, Scott L. Thomson, Scott D. Sommerfeldt, Kent L. Gee Gee (Brigham Young Univ., N-281A ESE Provo, UT 84602, crd26@byu.edu), Cole L. Duke, and David W. Krueger (Brigham Young Univ., Provo, UT 84602)

The use of error sensors in the near field of an axial fan can be used to achieve global attenuation of the blade passage frequency and its harmonics. The pressure field produced by the minimized sound power radiation of the fan and control source configuration dictates possible locations of the error sensors by creating pressure nulls in the near field. By minimizing pressure at these locations, the minimized sound power field can be reproduced. Near field locations can be further investigated using qualitative flow visualization techniques including smoke visualization and quantitative particle image velocimetry (PIV). Analysis of the flow field will help to understand the presence of turbulent flow at the error sensors which can be a cause of a decreased signal to noise ratio. Optimal locations as well as mounting techniques for the error sensors will be discussed based on the flow field analysis. The effects of active noise control on the flow field produced by the fan will also be discussed

\section{$4: 45$}

4pNS10. Further developments in the active control of free-field sound using near-field energy-based error signals. Ryan T. Chester and Timothy W. Leishman (Phys. Dept., Brigham Young Univ., N283 ESC, Provo UT 84602)

Practical efforts to actively control sound often require error sensors located in the acoustic or geometric near field of sound sources. Unfortunately, when using conventional acoustic pressure sensors, control performance often becomes very sensitive to sensor location. Optimal sensor positions are difficult to ascertain or maintain with changing system conditions. Alternate types of error sensors often lead to fewer positiondependent complications, producing global sound field control with greater ease and consistency. The distinct spatial and spectral uniformities of potential, kinetic, and total energy density for near-field error signal measurements will be discussed for the sound control of sources located in a free field. Numerical results will be compared to experimentally measured results. 


\title{
Session 4pPA
}

\section{Physical Acoustics: Bubbles, Modes, and Scattering}

\author{
Mark S. Wochner, Chair \\ Univ. of Texas, Applied Research Lab., 10000 Burnet Rd., Austin, TX 78758
}

Chair's Introduction-1:40

\section{Contributed Papers}

$1: 45$

4pPA1. Sonoluminescence from transient cavitation at high pressures in water and acetone. D. Felipe Gaitan and Ross A. Tessien (Impulse Devices, Inc. 13366 Grass Valley Ave., Grass Valley, CA 95945)

In highly degassed, clean liquids, transient acoustic cavitation can be triggered by fast neutrons, a phenomenon that has been known since $\sim 1960$ 's. The kinetic energy acquired by the nuclei allows it to ionize a small volume $(\sim 100 \mathrm{~nm}$ dia.) in the liquid, creating a vapor cavity that would normally last a couple of microseconds. If the acoustic amplitude and phase are right, this cavity expands by several orders of magnitude ( $\sim 500$ to 1500 microns dia.) and then collapses, emitting a short flash of light (1 to $40 \mathrm{nsec}$ ). The bubble continues to expand and collapse for several hundred cycles, eventually evolving into a larger (2 to $6 \mathrm{~mm}$ dia.) bubble cloud which lasts several milliseconds, depending on the conditions. The time duration of the light pulses is longer and their amplitude larger than those of single bubble sonoluminescense in water $(\sim 100$ to $300 \mathrm{psec}, 10^{5}$ photons/flash). The amplitude and time evolution of the light flashes has been analyzed as a function of the driving conditions and compared with computer simulations in an effort to infer the maximum plasma temperatures and densities, and perhaps the presence of shock waves, in the cavities.

\section{2:00}

4pPA2. Sonoluminescence: Theoretical explanation and comparison to experiment pertaining to the transduction of sound into radiated light. Harvey Woodsum (Sonetech Corp., 10 Commerce Park North, Unit 1, Bedford, NH 03110)

The subject of sonoluminescence, i.e., the transduction of sound into light via the intermediate step of cavitation bubble collapse, is of general interest and has sparked a great deal of published research. The present work considers the integration of bubble dynamics with the nonlinear generation of light via the Euler-Heisenberg theory [W. Heisenberg and $\mathrm{H}$. Euler, Z. Phys. 98, 714 (1936)], in relation to Schwingers' conjecture that bubble cavitation is "squeezing light from the quantum vacuum" via the Casimir effect [Schwinger, Proc. Natl. Acad. Sci., USA 90, 4505-4507, (1993)]. Preliminary results of applying this model appear to show good agreement with both radiated spectral shape and efficiency of sonoluminescent light production, as presented by the relevant literature.

\section{2:15}

4pPA3. Effects of liquid compressibility on coupled radial and translational motion of a bubble. Todd A. Hay, Mark F. Hamilton, Yurii A. Ilinskii, and Evgenia A. Zabolotskaya (Appl. Res. Labs., Univ. of Texas, Austin, TX 78713-8029)

In shock wave lithotripsy, explosive bubble growth is followed by a collapse phase during which bubble wall velocities can approach the speed of sound in the liquid. If the bubble is simultaneously in translation, the velocity of translation can also exhibit a very large spike during rebound. The velocity spike corresponds to conservation of translational momentum as the bubble radius, and thus entrained mass, become very small. It can then be important to include effects of compressibility on both the radial and translational motion. This presentation discusses corrections to the coupled equations for radial and translational motion when compressibility, and therefore acoustic radiation, are taken into account. For pure radial motion, compressibility introduces a radiation term proportional to the third time derivative of bubble volume, and inversely proportional to sound speed. For pure dipole motion, linear theory predicts a radiation force proportional to the second time derivative of the translational velocity, also inversely proportional to sound speed [Morse and Ingard, Theoretical Acoustics (1968)]. Numerical simulations will be presented that illustrate the magnitudes of compressibility effects on solutions of the coupled equations for radial and translational motion. [Work supported by the NSF Graduate Research Fellowship.]

2:30

4pPA4. Excitation of shape oscillation of bubble(s) in water under ultrasonic vibration. Ichiro Ueno, Tatsunori Kojo, and Shuhei Katase (Tokyo Univ. of Sci., 2641 Yamazaki, Noda, Chiba 278-8510, Japan)

Behavior of a single or multiple bubbles of $\mathrm{O}(1 \mathrm{~mm})$ in diameter under ultrasonic vibration was investigated experimentally. Excitations of surface wave and shape oscillation with distinct mode number were especially focused. Interaction between/among oscillating bubbles in a row rising in water was also introduced by considering bubbles volume variation during the oscillation. Through a series of the present experiment, variation of distinct mode number for shape oscillation as a function of the bubble radius is indicated.

\section{2:45}

4pPA5. Excitation of a vertical liquid capillary jet waveguide by modulated ultrasonic radiation pressure. J. B. Lonzaga, D. B. Thiessen, and P. L. Marston (Phys. and Astron. Dept., Washington State Univ., Pullman, WA 99164-2814)

The excitation of a liquid capillary jet issuing from a nozzle is investigated using internally applied modulated ultrasonic radiation pressure. The transducer used here is more efficient than one used in prior studies [J. B. Lonzaga et. al., J. Acoust. Soc. Am. 116, 2598 (2004)] and is suitable for nozzle velocities as low as $25 \mathrm{~cm} / \mathrm{s}$. At low velocities, the liquid jet is significantly tapered. As a consequence of the taper, the acoustic cutoff frequency increases with increasing distance from the nozzle. For ultrasound propagation down the jet from the nozzle, finite-element calculations of the radiation pressure show that the radial stress at the cutoff location is significantly larger than at any other region of the jet. Two distinct capillary modes exist and are conjectured to be excited near the cutoff location by modulated radiation pressure: one traveling upward and the other traveling downward. In a certain range of modulation frequencies, the latter is an exponentially growing mode and leads to the forced disintegration of the jet into liquid drops. Excitation of the growing mode at the nozzle is achieved for low-speed jets by using carrier frequencies close to or below the nozzle cutoff frequency. [Work supported by NASA.] 
bounded beams. It is shown that the Rayleigh waves themselves are the physical origin of the transmission of Rayleigh waves and not the scatter-

4pPA6. Scholte-Stoneley wave generation by means of diffraction on a corrugated surface: Influence of corrugation shape. Nico F. Declercq (Woodruff School of Mech. Eng., GATech, 801 Ferst Dr., Atlanta, GA 30332-0405, nico.declercq@me.gatech.edu and GT-Lorraine, 2 r. Marconi, 57070 Metz, France) and Bart Sarens (Katholieke Universiteit Leuven, Celestijnenlaan 200D, B-3001 Leuven, Belgium)

Because of the growing number of applications of phononic crystals and other periodic structures, there is a renewed and growing interest in understanding the interaction of ultrasound with periodically corrugated surfaces. A theoretical investigation is presented of the transformation of ultrasound incident from the solid side onto a solid-liquid periodically corrugated interface. It is shown that it is possible to tailor the shape of a corrugated surface with given periodicity such that there is a more significant amount of energy transformed into Scholte-Stoneley waves than if pure saw-tooth or sine-shaped surfaces were used. This permits the fabrication of periodic structures that can be patched on or engraved in body parts of a construction and enables efficient generation of ScholteStoneley waves. The study is performed for incident homogeneous plane waves as well as for bounded beams. Incident longitudinal waves are studied and also incident shear waves.

\section{3:15-3:30 Break}

\section{$3: 30$}

4pPA7. Nonlinear shear wave beams. Mark S. Wochner, Mark F. Hamilton, and Evgenia A. Zabolotskaya (Appl. Res. Labs., Univ. of Texas, P.O. Box 8029, Austin, TX 78713-8029, mwochner@arlut.utexas.edu)

The theoretical framework developed previously for plane nonlinear shear waves [Zabolotskaya et al., J. Acoust. Soc. Am. 116, 2807-2813 (2004)] has been extended to include diffracting beams. Two coupled KZK-type equations with cubic nonlinearity are obtained for the two transverse components of the particle velocity vector. The coupled nonlinear equations are solved numerically in the time domain using a nonoscillatory numerical scheme. Both linearly and elliptically polarized beams are considered. For circular polarization the equations decouple and become linear. Waveforms along the beam axis are presented that illustrate shock formation and coupling of the two velocity components for different polarizations. Beam patterns are presented showing the angular dependence of the nonlinearly generated harmonics. Because the evolution equations are derived using the parabolic approximation for narrow beams, the predicted beam patterns are axially symmetric for all polarizations whenever the amplitude and phase of the source are axially symmetric. Comparisons are made with the corresponding results obtained from the quadratically nonlinear KZK equation for sound beams in fluids. [Work supported by NIH and ARL IR\&D.]

\section{$3: 45$}

4pPA8. Leaky Rayleigh waves propagating around the corner of a thick solid plate: Finite element analysis. Ebrahim Lamkanfi, Nico F. Declercq (Woodruff School of Mech. Eng., GATech, 801 Ferst Dr., Atlanta, GA 30332-0405, nico.declercq@me.gatech.edu and GT-Lorraine, 2 r. Marconi, 57070 Metz, France), Wim Van Paepegem, and Joris Degrieck (Ghent Univ., Sint-Pietersnieuwstraat 41, 9000 Ghent, Belgium)

Finite element simulations reveal the experimentally indicated fact [Declercq et al, J. Appl. Phys. 96(10), 5836-5840 (2004)] that leaky Rayleigh waves propagating along the horizontal surface of a thick fluidloaded solid plate are transmitted around the corner of the solid plate. The experiments indicate that leaky Rayleigh waves are generated around the corner of the plate, but the experimental evidence is not fully conclusive whether the effect is caused merely by incident Rayleigh waves on the upper surface or by scattering effects when the incident beam interacts with the corner. The current study first investigates the reported experiments by means of the finite element method. Then the model is applied to study leaky Rayleigh waves separately from the incident and reflected ing effects caused by the incident bounded beam interacting with the edge of the plate.

\section{4:00}

4pPA9. Non-contact and local characterization of thin plates and cylindrical shells using the zero-group velocities (ZGV) Lamb modes. Dominique Clorennec, Claire Prada, Daniel Royer (Lab. Ondes et Acoustique, ESPCI, Université Paris 7 Denis Diderot, UMR CNRS 7587, 10 rue Vauquelin, 75231 Paris cedex 05, France), and Todd Murray (Boston Univ., Boston, MA 02215)

The group velocity of the symmetrical $S_{1}$ Lamb mode vanishes if the Poisson's ratio $v$ is smaller than 0.45 . At this $\mathrm{ZGV}$ point, the frequency $\times$ thickness product undergoes a minimum and two modes $\left(S_{1}\right.$ and $\left.S_{2 b}\right)$ propagate with opposite phase velocities. In our experiments, this ZGV mode is generated by a 10-ns laser pulse and detected at the same point using an optical interferometer. The spectrum of the signal reveals an intense narrow peak corresponding to the $S_{1}-Z G V$ Lamb mode resonance. A similar phenomena occurs for the $\mathrm{A}_{2}$ Lamb mode if $v<0.31$. The minima of the frequency $\times$ thickness product of the $S_{1}$ and $A_{2}$ modes only depend on $v$. The ratio of the resonance frequencies of these two modes is independent of the thickness. Thus, this experimental configuration allows a local and absolute measurement of $v$. Knowing $h$, the measurement of these resonance frequencies provides the bulk waves velocities with a high accuracy. This non-contact method was tested on different plates and shells. The same behaviour is observed in a cubic silicon crystal. Moreover in such anisotropic plates, we show that the resonance frequencies can be selected using a line laser source. These frequencies are well predicted by the theoretical dispersion curves.

\section{$4: 15$}

4pPA10. Periodic orbit techniques for mode shape approximation. Christopher J. Ham and M. C. M. Wright (Inst. of Sound and Vib. Res., Univ. of Southampton, University Rd., Southampton, UK SO17 1BJ, ch@isvr.soton.ac.uk)

At high frequency, numerical methods for calculating mode shape functions in a waveguide are expensive. The effect of modifying the shape of a waveguide in an industrial design context may only be calculated on a case-by-case basis if numerical methods are used. Periodic orbits are closed, repeating ray paths. A significant body of research in quantum physics indicates that periodic orbits provide fundamental insight into mode theory, which can be applied to acoustics. A method to approximate mode shape functions around short periodic orbits proposed by Vergini [J. Phys. A: Math. Gen. 33, 4709-4716, (2000)] and Babic and Buldryrev ["Short-Wavelength Diffraction Theory" (Springer, Berlin) (1991)] is implemented in this paper. In particular, mode shape functions in nonintegrable waveguides are approximated. This method links the geometry of the waveguide to the mode shape functions. The technique is extended to waveguides that have partly chaotic ray dynamics and is applied to the oval and eccentric annulus waveguides. The approximate mode shape functions from these two cases are compared with numerical results.

\section{$4: 30$}

4pPA11. Wigner distribution of an ultrasonic transducer beam pattern through a liquid-solid interface. Goutam Ghoshal and Jospeh A. Turner (Dept. of Eng. Mech., Univ. of Nebraska-Lincoln, W317.4 Nebraska Hall, Lincoln, NE 68588-0526)

Diffuse ultrasonic backscatter techniques are useful for probing heterogeneous materials to extract microstructural parameters and detect flaws that cannot be detected by conventional ultrasonic techniques. Such experiments, usually done using a modified pulse-echo technique, utilize the spatial variance of the signals as a primary measure of microstructure. Quantitative ultrasonic scattering models include components of both transducer beams as well as microstructural scattering information. Of particular interest for interpretation of many experiments is the propaga- 
tion through a liquid-solid interface. Here, the Wigner distribution of the beam pattern of an ultrasonic transducer through a liquid-solid interface is used in conjunction with the Bethe-Salpeter equation to model this scattering problem. The Wigner distribution represents a distribution in space and time of the spectral energy density as a function of wave vector and frequency. The general scattering model to be discussed encompasses the full multiple scattering behavior that occurs within the solid, while a simple Gaussian beam is used to model the transducer beam pattern. A simplified model restricted to the single-scattering regime is then compared with experimental results for materials of common interest. These results are anticipated to impact ultrasonic nondestructive evaluation and characterization of heterogeneous media.

\section{4:45}

4pPA12. Multiple scattering by a random configuration of cylinders in a poro-elastic medium: Properties of the coherent waves. Francine Luppé, Jean-Marc Conoir, and Sébastien Robert (LAUE, UMR CNRS 6068, Université du Havre, place R. Schuman, 76610 Le Havre, France)

An extension of Twersky's formalism is developed in order to obtain the dispersion equation of the coherent waves in a poro-elastic medium in which cylindrical scatterers are randomly placed. The high frequency regime only, where no dispersion effects occur in the absence of scatterers, is investigated. The scatterers lay within a slab-like region of the host medium, and an incident harmonic plane wave gives rise to coherent plane waves in the slab. At normal incidence, the coherent waves are damped, while they are inhomogeneous at other incidence angles. The dispersion equation obtained shows that the longitudinal (fast and slow) coherent waves are coupled, while the shear one obeys the Waterman and Truell's well-known dispersion equation.
5:00

4pPA13. Fresnel approximations for acoustic fields of rectangularly symmetric sources. T. Douglas Mast (Dept. of Biomed. Eng., Univ. of Cincinnati, 231 Albert Sabin Way, Cincinnati, OH 45267-0586)

A general approach is presented for determining the acoustic fields of rectangularly symmetric, baffled, time-harmonic sources under the Fresnel approximation. This approach is applicable to a variety of separable source configurations, including uniform, exponential, Gaussian, sinusoidal, and error function surface velocity distributions, with and without focusing in either surface dimension. In each case, the radiated field is given by a formula similar to that for a uniform rectangular source, except for additional scaling of wave number and azimuthal distance parameters. The expressions presented are generalized to three different Fresnel approximations that correspond respectively to diffracted plane waves, diffracted spherical waves, or diffracted cylindrical waves. Numerical results, for several source geometries relevant to ultrasonic applications, show that these expressions accurately depict the radiated pressure fields, except for points very near the radiating aperture. Highest accuracy near the source is obtained by choice of the Fresnel approximation most suited to the source geometry, while the highest accuracy far from the source is obtained by the approximation corresponding to diffracted spherical waves. The methods are suitable for volumetric computations of acoustic fields including focusing, apodization, and attenuation effects.

\title{
Session 4pPP
}

\section{Psychological and Physiological Acoustics: Environmental Sound Research, Spectral Integration, Cochlear Implants, and More (Poster Session)}

\author{
Marjorie R. Leek, Chair \\ Portland VA Medical Center, 3710 SW U.S. Veterans Hospital, Portland, OR 97207
}

\section{Contributed Papers}

\begin{abstract}
All posters will be on display from 1:30 p.m. to 4:30 p.m.. To allow contributors an opportunity to see other posters, contributors of odd-numbered papers will be at their posters from 1:30 p.m. to 3:00 p.m. and contributors of even-numbered papers will be at their posters from 3:00 p.m. to 4:30 p.m.
\end{abstract}

4pPP1. Naturalistic auditory scene analysis in children and adults. Robert Leech, Fred Dick, Jennifer Aydelott (School of Psych., Birkbeck, Univ. of London, London, UK, WC1E 7HX), and Brian Gygi (East Bay Inst. for Res. and Education, Martinez, CA)

In order to make sense of natural auditory environments, the developing child must learn to "navigate" through complex auditory scenes in order to segment out relevant auditory information from irrelevant sounds. This study investigated some of the informational and attentional factors that constrain environmental sound detection in auditory scenes, and how these factors change over development. Thirty-two children (aged 9-12 years) and 16 adults were asked to detect short target environmental sounds (e.g., a dog barking) within longer environmental background sounds (e.g., a barn) presented dichotically. The target environmental sounds were either congruent (i.e., normally associated with the background) or incongruent. Subjects heard either a single background presented to both the ipsilateral and contralateral ears or else different background sounds presented to the different ears. Results indicate that children's, but not adults' target detection is substantially less accurate when listening to two auditory scenes than to a single scene presented in both ears, suggesting a developmental shift in listeners' abilities to process multiple information streams. Interestingly, both children and adults find 
incongruent sounds more salient than congruent sounds. Furthermore, this incongruence "pop-out" effect is greatly reduced in the presence of the dual background (informational masking) condition.

4pPP2. Noise quality of traffic noise. Klaus Genuit, Sandro Guidati, Sebastian Rossberg, and Andre Fiebig (HEAD acoustics GmbH, Ebertstrasse 30a, 52134 Herzogenrath/Germany, klaus.genuit@head-acoustics.de)

Traffic noise is considered as one major source of noise annoyance in urban context indicated by the increasing number of complaints. The neglect of quality parameters regarding pass-by noise appears as a basic cause for the unreduced annoyance. Several parameters besides the SPL have an important influence on noise evaluation, such as low frequency contributions, temporal aspects or noise patterns. The European research project Quiet City Transport (6FP PL516420) was motivated by the EU Noise Directive 2002/49/EC and is dealing with vehicle pass-by noise and its evaluation in order to identify promising noise mitigation measures. In this framework a quantitative description of noise annoyance using psychoacoustic descriptors was achieved. By means of the detected metric certain perceptual-relevant aspects, such as the difference between diesel and gas, can be reconstructed. Furthermore, the development of a synthesis tool makes the simulation and binaural auralization of pass-by noise, traffic noise as well as specific vehicle noise sources possible. Based on the presented technique the detailed investigation of causes for noise annoyance is carried out. The results will be presented.

4pPP3. Sound source identification by compressive sensing. Raman Arora (Dept. Elec. and Computer Eng., Univ. of Wisconsin, Madison, WI 53705, ramanarora@wisc.edu) and Robert A. Lutfi (Univ. of Wisconsin, Madison, WI 53705, ralutfi@wisc.edu)

Compressive sensing (CS) is an emergent technology that has found increasing application in the areas of broadband signal monitoring and image reconstruction [D. Donoho, IEEE Trans. Info. Theory 52, 12891306 (2006)]. It involves a novel approach to sampling in which the salient information in signals is recovered from the projection of observations onto a small set of randomly-selected basis functions. The major attraction of CS is its capacity for accurate reconstruction based on sparse sampling and little or no prior knowledge of signals. This feature makes it attractive, as well, for application to the problem of sound source identification; an allied task in which sound waveforms as signals are to be classified according to their generating source. In the present paper CS is applied to examples of sound source identification tasks taken from the human psychoacoustics literature. The examples are used to demonstrate in these cases potential advantages of CS classification over traditional decision algorithms that sample at the Nyquist rate. Parallels to the human data are also noted, entertaining speculation as to the role CS classification might play in theoretical thinking about human sound source identification. [Research supported by NIDCD grant 5R01DC006875-02.]

4pPP4. Cochlear implant-mediated perception of non-linguistic sounds. Yell Inverso, Corinne Bickley (Gallaudet Univ., Dept. of Hearing and Speech Lang. Sci., Washington, DC), and Charles Limb (Johns Hopkins Hospital, Baltimore, MD)

Cochlear implants $(\mathrm{CI})$ have provided sound perception to profoundly deaf individuals, revolutionizing the treatment of sensorineural hearing loss. Thus far, the overwhelming majority of test measures to assess implant candidacy, efficacy, and progress are based on speech perception. Non-linguistic speech (NLS) stimuli, by comparison, have received comparatively little attention, despite the importance of NLS sounds for environmental sound awareness. For the prelingually deafened population, in whom speech perception may never be fully realized, NLS perception is an especially critical area deserving further study. The specific aims of this study were to determine the accuracy with which postlingually deafened CI users perceive NLS, and to determine if there is an association between acoustic characteristics of the NLS and ability to identify these sounds using a CI. We used five main categories of NLS: human vocal non- linguistic, animal/insect, nature/ambient, mechanical/alerting, and musical to comprise a test battery that was given to 10 postlingually deafened CI users. Results of the pilot study showed overall poor ability of CI users to recognize NLS. Furthermore, an association between speech perception and accuracy of NLS was identified. Our results suggest that NLS is a difficult category of complex sound for CI users to perceive.

4pPP5. Spacial cognitive timbre dimensions of physical modeling sounds using multi-dimensional scaling (MDS) techniques. Rolf Bader (Inst. of Musicology, Univ. of Hamburg, 20354 Hamburg, Germany)

The cognitive spacial dimensionality of timbre in the minds of listeners is investigated. Physical models of strings, rods, plates, and membranes and couplings of these obtaining simple musical instruments are used. Here, for each model, eight sounds have been produced by linear change of only one parameter of the physical model. So, i.e., the change of inharmonicity in a string sound modeled as coupling of the string differential equation with a bending stiffness equation by linearly increasing the bending stiffness is investigated. After the judgment procedure, the mean judged similarities of all subjects are fit into an $n$-dimensional space. The spacial dimensions then have to be interpreted in terms of the physical changes of the sounds distributed over those dimensions. It could be shown that linear changes in inharmonicity of strings do have both, a linear perception as well as sudden phase changes, a finding relevant to psychoacoustic noise perception or room acoustics. Also, the problem of changing plate thickness, different striking points of membranes, or coupling of snare membranes to their vessels with changing cutoff angle of the vessel is investigated. Furthermore, just noticeable differences with timbre changes as well as judgments of very different sounds are discussed.

4pPP6. Practical kurtosis-based blind recovery of a speech source in real-world noise. Matthew D. Kleffner and Douglas L. Jones (Coordinated Sci. Lab., Univ. of Illinois, 1308 West Main St., Urbana, IL 61801, kleffner@uiuc.edu)

In many multi-sensor, single-source applications noise interferes with cleanly recovering the source. Various approaches have been designed to recover sources in interference, but most of them require prior knowledge or assumptions that limit their applicability to real-world environments. We present a novel and practical frequency-domain method for blindly recovering a single, nonstationary, high-kurtosis source in arbitrary lowkurtosis interference using a narrowband kurtosis objective. This method handles convolutional mixing, does not impose a theoretical limit on the number of interferers, and relies only on the kurtosis properties of the source and interference. A kurtosis-based objective is used to compute instantaneous filter weights in each frequency bin, since linear combinations of speech with low-kurtosis interference tend to have lower kurtosis than speech alone. The set of bin-by-bin scale factors needed for the total spatio-temporal recovery filter are computed by using estimated steering vectors to recover the signal as it would sound at a particular sensor. Speech signal-to-interference ratio gains of 5 to $10 \mathrm{~dB}$ are demonstrated at low input SIRs in a variety of real-world situations in both a car and a reverberant room.

4pPP7. Amplification and training effects in resolution and crossspectral integration of redundant cues. Blas Espinoza-Varas (Commun. Sci. \& Disord., OUHSC, Oklahoma City, OK 73190), Mohamed Bingabr (UCO, Oklahoma City, OK 73034), and Philip Loizou (Univ. of Texas, Dallas, TX 75083)

Speech recognition of hearing-aid and cochlear-implant users is constrained partly by inefficient integration of information conveyed by multiple frequency channels: owing to cross-channel interference, multichannel recognition accuracy is often lower than the sum of single-channel 
accuracies, especially if one channel has impaired sensitivity. With complex tones consisting of a mid- $(\mathrm{M})$ and a high-frequency $(\mathrm{H})$ component $(1000$ and $3127 \mathrm{~Hz}$ ), the resolution and cross-spectral integration (CSI) of simultaneous increments in $\mathrm{M}$ frequency (IFM) and $\mathrm{H}$ duration (ITH) were studied as a function of $\mathrm{H}$ amplification and training; $\mathrm{M}$ and $\mathrm{H}$ impinged, respectively, on normal- and impaired-sensitivity regions of listeners with sensorineural losses. Without amplification, ITH resolution was negligible, the IFM discrimination threshold did not decrease with ITH, and CSI was nil. With $\mathrm{H}$ amplification, resolution improved moderately for ITH but decreased for IFM; that is, increasing $\mathrm{H}$ audibility interfered with IFM resolution. As a result, the IFM discrimination threshold decreased with ITH but CSI was inefficient and remained so after extensive training. However, ITH resolution and CSI efficiency improved significantly by training listeners to attend to $\mathrm{H}$ and respond only on the bases of ITH. The improvement associated with $\mathrm{H}$ amplification increased significantly with high-frequency listening training. [Work supported by PHF.]

4pPP8. A correlation feedback model of audio-visual integration. Sheena Luu and Willy Wong (IBBME, Rosebrugh Bldg., 164 College St., Rm. 407, Univ. of Toronto, Toronto, ON, M5S 3G9, Canada)

To date, a lot of empirical research has been done by many laboratories to investigate how information from different senses interacts to affect perceptual experience. Given the quantity and variety of observations published, it is necessary to begin to organize and integrate the information into a coherent framework. Here a framework is presented in the form of a systems-level model of auditory-visual interaction in the brain. The temporal correlation feedback model is a computational model to illustrate the change in human experience and perception of auditory and visual information as a result of the interaction of the two senses. The model is able to explain many of the effects of audiovisual integration and the characteristics of audiovisual synchrony detection observed in other studies.

4pPP9. Spectral coherence predicts perceptual resilience of speech to temporal distortion. Christian E. Stilp, Joshua M. Alexander, and Keith R. Kluender (Dept. of Psych., Univ. of Wisconsin-Madison, 1202 W. Johnson St., Madison, WI 53706)

Speech perception is remarkably resilient to signal perturbation. For example, Saberi and Perrott [Nature, 398, 760 (1999)] found that sentences in which successive segments as long as $130 \mathrm{~ms}$ have been temporally reversed are relatively intelligible. They suggested that ultra-lowfrequency modulation envelopes $(3-8 \mathrm{~Hz}$ ) are critical to intelligibility. We tested whether information transmitted, not physical units (e.g., ms, Hz), better explains performance. Listeners heard a large cohort of synthesized, seven-syllable sentences at three different speaking rates $(2.5,5.0,10$ syllables/second) with time-reversed segments of multiple durations (20, $40,80,160 \mathrm{~ms})$. Intelligibility decreased as duration of time-reversed segments increased, decreasing more slowly for slower (longer) sentences. When intelligibility is plotted to reflect proportion of message (not time) that was time reversed, data for all three rates collapse onto a single curve. To estimate potential information across time, we used spectral coherence to quantify the degree to which successive portions of the signal are similar to, or can be predicted from, a given spectral slice with no prior expectations such as knowledge of speech or language. Spectral coherence alone accounts substantially for performance. Information transmission, not physical measures of time or frequency, is more useful for understanding perception of speech. [Work supported by NIDCD.]
4pPP10. Modulation interference in speech recognition by cochlear implant users. Bom Jun Kwon (Dept. of Commun. Sci. and Disord., Univ. of Utah, Salt Lake City, UT 84112) and Peggy B. Nelson (Univ. of Minnesota, Minneapolis, MN 55455)

When listening to speech in a background masker, normal-hearing listeners take advantage of envelope fluctuations or dips in the masker (or the moments that the SNR is instantaneously favorable). Cochlear implant listeners, however, do not exhibit such ability. In a previous study [Nelson et al., J. Acoust. Soc. Am. 113, 961-968 (2003)], speech recognition performance with modulated maskers was similar or slightly worse than with a steady noise. Clinical observation indicates that implant listeners usually have more difficulty understanding speech in modulated backgrounds. In the present study, the recognition of IEEE sentences by Nucleus recipients was measured in a variety of backgrounds. In tightly controlled conditions via direct stimulation, performance is often substantially poorer with modulated backgrounds (the differences in score are as large as 20-30\%), strongly indicating that they are subject to modulation interference [B. J. Kwon and C. W. Turner, J. Acoust. Soc. Am. 110, 1130-1140 (2001)]. A mixture of speech and modulated backgrounds might be perceived as one distorted signal, rather than two signals, compromising the identity of the speech, as perceptual segregation of signals is very challenging. A future implant system should incorporate a strategy to provide multiple input sounds without sacrificing the sound identity.

4pPP11. Simulating bipolar and monopolar cochlear-implant stimulation in speech recognition by normal-hearing listeners. Mohamed Bingabr (Dept. of Phys. \& Elec. Eng., UCO, Oklahoma City, OK 73034), Blas Espinoza-Varas (OUHSC, Oklahoma City, OK 73190), Philip C. Loizou (Univ. of Texas, Dallas, TX 75083), and Kenneth Hawkins (UCO, Oklahoma City, OK 73034)

An algorithm was developed to simulate cochlear-implant (CI) bipolar and monopolar stimulation (BS and MS) in speech recognition by normalhearing listeners. In MS, the active electrode is inside and the return is outside the cochlea, and electrical current decays at $0.5 \mathrm{~dB} / \mathrm{mm}$. In BS, both electrodes are adjacent inside the cochlea and current decays at 4.0 $\mathrm{dB} / \mathrm{mm}$ to either side of the active electrode [Bruce et al., IEEE Trans. Biomed. Eng. 46, 617 (1999)]. Owing to the faster decay rate, spatial (spectral) resolution is higher with BS than with MS. In recognition of CNC sentences, decay rate was simulated as spectral smearing. Speech was processed through 6,11 , or 22 pass bands, rectified, and low-pass filtered $(200 \mathrm{~Hz})$. The passbands amplitude envelopes modulated the amplitude of noise bands equal in center frequency to the passbands. The spectral envelope of each noise band decayed to either side of the center frequency at a rate matching the MS or BS current decay (i.e., noise-band bandwidth was proportional to decay rate). With the summed noise bands, recognition was studied as a function of number of pass bands. [Work supported by NIH.]

4pPP12. Speech intelligibility in noise with single-microphone noise reduction implemented in 9-, 16-, and 24-channel compressors. Michael J. Nilsson, Michelle L. Hicks, Robert M. Ghent, Jr., and Victor H. Bray, Jr (Sonic Innovations, Auditory Res. Lab, 2795 East Cottonwood Pkwy., Ste. 660, Salt Lake City, UT 84121)

Reception Threshold for Sentences as measured by a modified version of the HINT test [M. J. Nilsson et al., J. Acoust. Soc. Am. 95(2), 10851099 (1984)] were collected on hearing-impaired listeners fit binaurally with digital hearing aids incorporating a 9-channel, 16-channel, and 24channel spectral subtraction-like technique of single-microphone noise reduction. Thresholds were measured in noise presented in a twodimensionally diffuse soundfield with the subjects listening unaided, aided without noise reduction, and aided with noise reduction. The ability of noise reduction to reduce the level of steady state noise was quantified using $2 \mathrm{~cm} 3$ coupler measures with flat linear fittings (to measure the maximum attenuation possible) as well as using each of the listeners' prescribed fittings (to better relate to the changes in performance measured 
in the sound field). Previously measured benefit from noise reduction [(M. J. Nilsson et al., J. Acoust. Soc. Am. (2000)] is hypothesized to occur from frequency-specific gain manipulations that increases gain in frequencies where speech is the dominant signal and decreases gain in frequencies where noise is the dominant signal. Additional benefits from smaller, independent channel structures are therefore expected from the 16 and 24 channel systems.

4pPP13. Validation of the noise reduction index (NRI) as an estimate of the signal-to-noise ratio change of a mixed signal by a hearing aid. Robert M. Ghent, Michael J. Nilsson, and Victor H. Bray (Sonic Innovations, Inc., Auditory Res. Dept., 2795 E. Cottonwood Pkwy., Ste. 660, Salt Lake City, UT 84121)

The NRI is an extension of an idea discussed by Licklider [J. Acoust. Soc. Am. 20, 150-159 (1948)] and implemented in practice by Hagerman and Olofssen [Acta Acoustica 90, 356-361 (2004)] to investigate the conventional wisdom that asserts that a hearing aid cannot fundamentally alter (improve) the input SNR using a single-microphone signal processing algorithm such as digital noise reduction. The present research explores the validity of the NRI by using it to estimate the change in input SNR through a variety of audio devices, including linear hearing aids (with and without directional microphones), a directional microphone designed for noise rejection on a concert stage, an ear trumpet, and a multi-channel hearing aid with a digital noise reduction algorithm both engaged and disengaged. Results indicate that the change in SNR through an audio device can be negative as well as positive, that the NRI is a robust and valid method for estimating this change, and that single-microphone noise reduction algorithms can improve the input SNR when properly designed. The test setup and methodology, along with some pitfalls to avoid when performing these measurements, will be discussed.

4pPP14. Speech perception from a crudely quantized spectrogram: A figure-ground analogy. Douglas Brungart, Nandini Iyer, and Brian Simpson (Air Force Res. Lab., 2610 Seventh St., WPAFB, OH 45433, douglas.brungart@wpafb.af.mil)

Recent research into auditory chimeras has shown that comprehensible speech can be generated by superimposing the frequency-dependent modulation envelope of a target speech signal onto the fine structure of music, speech, or almost any other broadband waveform. In this experiment, we show that, in certain cases, intelligibility can be achieved with an even simpler stimulus that preserves only the rough outline of the energy distribution of the target speech waveform. This stimulus was generated by 1 ) dividing the target speech spectrogram into discrete tiles that were 1/3rd octave band wide and $7.8 \mathrm{~ms}$ long; 2) identifying the minimum number of tiles necessary to capture $90 \%$ of the energy in the target waveform; and 3) filtering a uniform, broadband noise to activate only those tiles that met the threshold energy criterion in the original stimulus. The resulting stimulus captures the overall outline of the distribution of energy in the target speech, but contains no information about the relative distribution of energy within the spectrogram. In this paper, we discuss the use of this stimulus in a series of auditory figure-ground experiments where the target speech signal differs from the background by only a single parameter (relative level, ITD, ILD, etc.).

4pPP15. Transformation of live versus recorded speech from the mouth to the open or occluded ear. Dragana Barac-Cikoja, Linda Kozma-Spytek, and Stephanie Adamovich (Gallaudet Univ., 800 Florida Ave. NE, Washington, DC 20002, dragana.barac-cikoja@gallaudet.edu)

Auditory self-monitoring studies require the participants' speech feedback to be manipulated experimentally and then presented to the ear. The acoustic levels of the altered speech must be the same as those under normal speech feedback conditions when speech is transmitted to the ear live, directly from the mouth. Therefore, it is critical to understand the transfer function between the mouth and the eardrum. To further this understanding, live and played-back self-generated speech for two female and two male individuals were recorded using a probe tube microphone inserted into the ear canal at a fixed distance from the tragus. The playedback speech samples were delivered via a B\&K artificial mouth, at the sound pressure level calibrated to match the level at the lips. The artificial mouth was placed offset to the participants right side and in line with the $x$ and $z$ axes of his/her mouth position. Recordings were conducted with the ears either open or sealed with ER-3A insert earphones. In addition, the level and content of speech were systematically varied. The equivalent sound level and long-term average spectra of the corresponding pairs of speech samples were analyzed as a function of the experimental manipulations.

4pPP16. An active noise cancellation system to reduce the intense noise caused by magnetic resonance imaging. Deborah Hall, John Chambers (MRC Inst. of Hearing Res., University Park, Nottingham, NG7 2RD, UK), Kimberly Quinn (Nottingham Trent Univ., Nottingham, NG11 8NS, UK), and Michael Akeroyd (MRC Inst. of Hearing Res., Glasgow, G31 2ER, UK)

Magnetic resonance imaging (MRI) has become the dominant technique for noninvasive measurements of central auditory function in humans, but its operation generates intense acoustic noise (some 115 decibels). Although this can typically be attenuated by about 30 decibels with ear defenders, there is considerable scope for additional benefit. We took advantage of two features of the sound: (i) its predictable temporal onset, and (ii) its fixed power spectrum (which is dominated by intense harmonic components at low frequencies) to engineer an active noise cancellation (ANC) system to achieve further noise reduction. We measured its perceptual effectiveness by collecting detection thresholds for a tonal signal, masked by the image acquisition noise of the scanner (Philips 3 Tesla Intera) with and without the ANC operating. We presented signal frequencies centered at the peaks of the objective cancellation $(150,300$, and 600 $\mathrm{Hz}$ ), plus $450 \mathrm{~Hz}$ (a control frequency achieving no significant acoustic cancellation). Across four listeners, the ANC system gave average benefits of $4,11,15 \mathrm{~dB}$, respectively, at the peaks and $10 \mathrm{~dB}$ at $450 \mathrm{~Hz}$. These results confirm that the system offers substantial benefits for audibility in MR research. Moreover, the ANC equipment does not interfere with image quality.

4pPP17. Effect of level variation on intensity resolution in intensitydiscrimination and increment-detection paradigms. Walt Jesteadt and Donna L. Neff (Boys Town Natl. Res. Hospital, 555 N. 30th St., Omaha, NE 68131)

In studies of profile analysis, the level of the pedestal and increment have been varied together from interval to interval by up to $40 \mathrm{~dB}$ to reduce the likelihood that subjects could perform the task by comparing the overall level in the two observation intervals. This range of rove was chosen based on a calculation by Green [Profile Analysis (Oxford, New York, 1988)]. In the present study, adaptive thresholds for intensity resolution were obtained in intensity discrimination (pedestal and increment $100 \mathrm{~ms}$ ) and increment detection (pedestal $100 \mathrm{~ms}$, increment $300 \mathrm{~ms}$ ) paradigms as a function of the range of interval-to-interval level variation (0 to $50 \mathrm{~dB}$ in 10-dB steps). In roving conditions, the levels of pedestal and increment varied in parallel. In jittered conditions, only pedestal level varied from interval to interval. Increments were $2-\mathrm{kHz}$ sinusoids. Pedestals were $2-\mathrm{kHz}$ sinusoids or narrow-band noises centered on $2 \mathrm{kHz}$. Effects of level variation were greater in roving than in jittered conditions. Increment detection was unaffected by roving level, whereas thresholds for intensity discrimination were poorer than Green's best-case prediction by a relatively constant amount for all rove ranges. [Work supported by NIH-NIDCD.] 


\title{
Session 4 pSC
}

\section{Speech Communication: Vowel Production and Perception (Poster Session)}

\author{
Stephen M. Tasko, Chair \\ Western Michigan Univ., Speech Pathology and Audiology, 1903 W Michigan Ave., Kalamazoo, MI 49008-5355
}

\section{Contributed Papers}

\begin{abstract}
All posters will be on display from 1:30 p.m. to 4:30 p.m. To allow contributors an opportunity to see other posters, contributors of odd-numbered papers will be at their posters from 1:30 p.m. to 3:00 p.m. and contributors of even-numbered papers will be at their posters from 3:00 p.m. to 4:30 p.m.
\end{abstract}

4pSC1. Spatial-temporal patterns of vowel articulation: A crossspeaker study. Stephen Tasko (Dept. of Speech Pathol. and Audiol., Western Michigan Univ., 1903 W. Michigan Ave., Kalamazoo, MI 49008-5355, stephen.tasko@wmich.edu)

An important goal in speech production research is to establish a quantitative description of normal or typical articulatory behavior. This description should be expressed in a compact but meaningful way and be robust enough to generalize across a large number of speakers who differ in physical size, gender, age, etc. This is a significant challenge given the numerous degrees of freedom within the articulatory system and the range of speaker variability. However, Hashi et al. [J. Acoust. Soc. Am. 104, 2426-2437 (1998)] used x-ray microbeam data to develop a procedure for normalizing vocal tract size and shape. This procedure reduced crossspeaker variation in the articulatory postures of vowels. The authors limited their analysis to a single time slice at the acoustic steady state. There is little available information regarding speaker-general time-varying articulatory patterns associated with vowel production. This study will address this issue. Fifty-three healthy speakers from the University of Wisconsin $\mathrm{x}$-ray microbeam database served as subjects. The speech task consisted of productions of American English vowels in a $/ \mathrm{sVd} /$ context. Analysis focused on the movement of a number of vocal tract markers over the duration of each vowel. Vowel-specific spatial temporal patterns common to the speaker group will be described.

4pSC2. Predicting pharyngeal diameter from anterior tongue pellet positions in Swedish. Michel T-T. Jackson and Richard S. McGowan (CReSS LLC, 1 Seaborn Pl., Lexington, MA 02420)

One goal of phonetics is to quantitatively understand articulation in human speech production. As part of this goal, we are interested in the extent to which some vocal tract dimensions, specifically midsagittal pharyngeal diameters, are predictable from flesh points of the anterior tongue. In a reanalysis of $x$ rays of speakers producing Swedish vowels, we modified previous procedures [Whalen et al., J. Speech, Lang. Hear. Res. 42, 592-603 (1999); Nix et al., J. Acoust. Soc. Am. 99, 3707-3717 (1996)] to predict pharyngeal diameters from pseudo-pellet positions. We used principal component analysis to reduce the number of pellet degrees of freedom from eight to three prior to applying linear regression from these three independent variables to each of several dependent pharyngeal diameters. Except for the regions around the laryngopharynx and uvula, the pharynx dimensions predictable from linear regressions were significant at the $p<0.05$ level. Numerical experiments show that it is crucial to reduce the number of independent variables in tests of statistical significance. [Work supported by Grant No. NIDCD-001247 to CReSS LLC.]
4pSC3. Vowel identification and vowel space characteristics. Amy Neel (Dept. of Speech \& Hearing Sci., MSC01 1195, 1 Univ. of New Mexico, Albuquerque, NM 87131) and Jean Andruski (Wayne State Univ., Detroit, MI 48202)

Acoustic characteristics of ten vowels produced by 45 men and 48 women from the Hillenbrand et al. (1995) study were correlated with identification accuracy. Global (mean f0, F1 and F2, duration, and amount of formant movement) and distinctive measures (vowel space area, mean distance among vowels, f0, F1 and F2 ranges, duration ratio between long and short vowels, and dynamic ratio between dynamic and static vowels) were used to predict identification scores. Global and distinctive measures accounted for less than one-fourth of variance in identification scores: vowel space area alone accounted for $9 \%$ to $12 \%$ of variance. Differences in vowel identification across talkers were largely due to poor identification of two spectrally similar vowel pairs /ae/-/eh/ and /uh/-/ah/. Results of acoustic analysis and goodness ratings for well-identified and poorly identified versions of these vowels will be presented. Preliminary analysis revealed that well-identified vowels differed from poorly identified tokens not only in static formant frequencies but also in duration and amount of formant movement over time. A talker's ability to create distinctions among neighboring vowels is more important in determining vowel intelligibility than overall measures such as vowel space area.

4pSC4. Exploring the natural referent vowel hypothesis in infant perception experiments. Ocke-Schwen Bohn (English Dept., Arhus Univ., DK-8000 Arhus C, Denmark)

Directional asymmetries in infant vowel perception studies have led Polka and Bohn [Speech Commun. 41, 221-231 (2003)] to posit the Natural Referent Vowel hypothesis (NRV) according to which vowels that are relatively more peripheral in the F1/F2 space are perceptually privileged vis-a-vis less peripheral vowels. This bias has been observed in preference and in change/no change experiments with infants and with adult nonnative listeners. NRV was further tested in headturn (change/no change) experiments with Danish-learning infants. With one important exception, results support the NRV by showing that the predicted asymmetries exist in areas of the vowel space that have not been previously explored. The exception was observed for the Danish front vowel pair /e//ø/, where NRV incorrectly predicts that a change from the more peripheral /e/ to the less peripheral $/ \varnothing /$ is harder to discriminate than a change in the opposite direction. Interestingly, the unexpected asymmetry was observed only with infants up to the age of 7.5 months. Older infants showed a nonsignificant trend for an asymmetry in the opposite direction, as predicted by NRV. A modification of NRV to incorporate this finding will be proposed. [Research supported by Grant 25-01-0557 from the Danish Research Council for the Humanities (FKK).] 
4pSC5. Identification of brief vowel sequences by young and elderly listeners. Diane Kewley-Port, Larry Humes, and Daniel Fogerty (Dept. of Speech and Hearing Sci., Indiana Univ., Bloomington, IN 47405, kewley@indiana.edu)

Aging generally has a negative impact on temporal processing. Four experiments using vowel sequences are presented from a large series of temporal processing studies of age-related changes across three sensory modalities. These four experiments used adaptive tracking to determine the smallest temporal difference between the onsets of vowels (SOA) that preserved identification of either the vowel sequences or ear presented. Vowels in the four words (pit, pet, pot, put) from a male speaker were processed using STRAIGHT to have the same length $(70 \mathrm{~ms})$ and F0 (100 $\mathrm{Hz})$ and equated for rms level. Young $(N=40)$ and elderly $(N=40$; 60-79 years) adults identified the vowels in isolation with better than $90 \%$ accuracy. Preliminary analyses based on $40 \%$ of the data are summarized here. Baseline performance by young listeners in the monaural, two-vowel sequence task was $\mathrm{SOA}=20 \mathrm{~ms}$. For the other three conditions (monaural four-vowel sequences, dichotic two-vowel sequences, and dichotic twovowel sequences for ear presented), SOA values increased by three to four times. This pattern of SOA values was similar for the elderly listeners. However, as expected, SOA values were significantly longer for the elderly group, but only by a small percentage $(<30 \%)$. [Work supported by NIA R01 AG022334-02.]

4pSC6. The development of relative amplitude changes across acoustic discontinuities in nasal+vowel syllables. Meredith A. Oakey and Ralph N. Ohde (Vanderbilt Univ., Vanderbilt Bill Wilkerson Ctr., 1215 21st Ave. South, Rm. 8310, Nashville, TN 37232-8242)

In adult productions of nasal+vowel syllables, relative amplitude changes occur in various frequency regions across acoustic discontinuities and provide important cues as to the place of articulation of nasal consonants. The purpose of this study was to investigate the development of relative amplitude changes in children as potential acoustic correlates to place of articulation. Four age groups of eight participants each $(3,5,7$, adult) served as speakers. Participants produced five productions each of $\mathrm{CV}$ syllables comprised of $[\mathrm{m}]$ and $[\mathrm{n}]$ in the context of four vowels ([i æ $\mathrm{u} \mathrm{a}]$ ). These syllables were segmented into approximately $25 \mathrm{~ms}$ segments of the murmur and $25 \mathrm{~ms}$ segments of the vowel bordering the period of discontinuity. The relative amplitude changes in low- and high-frequency ranges from the murmur to the vowel were determined using summed amplitude information from fast Fourier transform (FFT) analyses. Previous research showed systematic differences in relative amplitude between $[\mathrm{m}]$ and $[\mathrm{n}]$ in adult syllable productions, but only marginal differences were observed in very young children's speech. The relative amplitude property, a landmark cue representing place of articulation of nasal consonants, is predicted to develop with age based on these preliminary analyses. [Work supported by NIH and an ASHA SPARC Award.]

4pSC7. An acoustical study of English constant-vowel (CV) syllables. Byunggon Yang (English Education Dept., Pusan Natl. Univ., 30 Changjundong Keumjunggu, Pusan 609-735, South Korea, bgyang@pusan.ac.kr)

This study examined acoustic characteristics of $900 \mathrm{CV}$ syllables produced by five English native speakers downloaded from http:// www.nsi.edu/users/patel/download.html. Those target syllables were produced quite synchronously between the syllable /ba/ twenty times. The syllables were segmented and normalized by the maximum intensity value of each syllable and were divided into consonant or vowel sections by a few visible acoustic criteria. Intensity values were collected at 100 relative time points per syllable. Also, cumulative intensity values and consonant and vowel durations along with the ratio of the onset consonant to each syllable were measured using Praat scripts. Results showed as follows: First, the consonantal section amounted to a quarter of the syllable in terms of both the cumulative intensity and duration. Second, the consonantal ratio by the cumulative intensity was similar to that by the duration.
Finally, the sum of the cumulative intensity values in each syllable partially coincided with the order of consonant groups on the current sonority scale while the vowel section of each syllable showed almost comparable intensity curve. Further studies would be desirable on more reliable acoustical measurements and sophisticated perceptual experiments on the English syllables.

4pSC8. Modeling vowel normalization and sound perception as sequential processes. Ricardo A. Hoffmann Bion and Paola Escudero (Inst. of Phonetic Sci., Univ. of Amsterdam, The Netherlands, escudero@uva.nl)

This study constitutes the first attempt at combining vowel normalization procedures with the linguistic perception framework of stochastic Optimality Theory and the Gradual Learning Algorithm (Boersma, 1998). Towards this end, virtual learners possessing different normalization procedures, and a control learner with no normalization procedure, were trained to perceive Brazilian Portuguese and American English vowels. The only parameters fed into the model were the first two formants of the vowels. The simulations assume that: (i) vowel normalization occurs prior to vowel categorization, (ii) each learner has acquired a different normalization algorithm at the time of the simulations, and (iii) perceptual learning takes place when mismatches between the word intended by the speaker and the word perceived by the virtual listener occur (Escudero \& Boersma 2004). Our results show that learners equipped with normalization algorithms outperformed the control learner, obtaining accuracy scores from 20 to $30 \%$ higher. When equipped with vowel-extrinsic procedures, learners managed to reach more than $90 \%$ of correct responses. Thus, a formal model in which normalization and sound perception are implemented as two sequential processes delivers the expected results, as it accurately models vowel perception even when the training and testing sets have speakers with different ages and gender.

4pSC9. The value of $\mathrm{F0}, \mathrm{F3}$, and $\mathrm{F} 4$ in identifying disguised voices. Jean E. Andruski, Nikki Brugnone, and Aaron Meyers (Dept. of Commun. Sci. \& Disord., 207 Rackham Bldg., 60 Farnsworth Ave., Detroit, MI 48202,ag0611@wayne.edu)

This study examines the value of F0, F3, and F4 for identifying speakers from a group of ten male and female speakers when the speakers deliberately changed their F0 and/or their articulatory patterns. The speakers were recorded producing a short passage in their normal speaking voice, a lower than normal speaking voice, and using a vocal disguise of their choice. Although F1 and F2 are strongly affected by articulation, the higher formants show relatively little effect of articulation. This relative stability may make them more useful than F1 and F2 for examining speaker identity. While formants reflect vocal tract cavity dimensions, F0 reflects a different aspect of speaker identity, namely the mass and stiffness of the speaker's vocal folds. Like F3 and F4, F0 shows relatively little effect from articulation. However, speakers can voluntarily shift F0 into a range that is different from what they normally use. Measurements of F0, F3, and F4 were taken on selected stressed vowels from each recording condition. Measurements from the normal voice condition were used to train a discriminant analysis, which then classified vowels from the low F0 and vocal disguise conditions by speaker. Accuracy of the classification results will be presented and discussed.

4pSC10. Tongue action in silence: Japanese voiceless vowels. Diana Archangeli, Adam Baker, Alina Twist, and Yuko Watanabe (Univ. of Arizona, Douglass Bldg., Rm. 200E, Tucson, AZ 85721, yukow@email.arizona.edu)

The study presents acoustic and articulatory analyses of the Japanese voiceless vowels [(i)] and [(u)] arguing Japanese listeners have internal representations for the vowels and perceive them from articulatory information while physical properties of vowels are absent. Ogasawara (2006) 
reports that there is no formant band in Japanese voiceless vowels. It is an open question as to whether there is an articulatory gesture associated with the voiceless vowels, and whether this gesture is the same or different as that of voiced vowels. To answer the question, we investigated production of voiceless vowels by a native Japanese speaker. We created 11 pairs of nonsense words such as [hogito] vs [hok(i)to] and [hoguto] vs [hok(u)to]. We recorded the speakers production of the stimuli with the voiceless vowels and their voiced counterparts using the ultrasound device to capture tongue images. The same recording was examined for acoustic analysis. An investigation of the ultrasound images reveals there are tongue gestures for the voiceless vowels, movements differ depending on which voiceless vowel it is, and the movements parallel those of the voiced counterparts. These results argue in favor of the view that Japanese speakers internal representations of words include a representation of distinct voiceless vowels.

4pSC11. Are vowels in African American English affected by Southern vowel shift? Yolanda F. Holt, Ewa Jacewicz, and Robert A. Fox (Speech Percept. and Acoust. Labs., Speech and Hearing Sci., Ohio State, 1070 Carmack Rd., Columbus, OH 43210-1002, holt.174@osu.edu)

There is a debate regarding whether the variety of speech called African American Vernacular English (AAVE) participates in any of the vowel shifts identified in today's American English. Because pronunciation of vowels in AAVE has never been studied with sufficient experimental rigor, it is unknown whether the vowel system of AAVE differs from those of closely-related regional variants of American English. The present experiment examines the apparent resistance of AAVE to assimilation into Southern American English (SAE) and seeks to determine the presence or absence of Southern Vowel Shift in the speech of speakers of AAVE. Acoustic measures of AAVE vowels produced by male speakers born and raised in Statesville, NC (Piedmont region) are compared with the same measures of SAE vowels spoken by European-American male speakers living in western North Carolina. Acoustic measures (obtained from fourteen monophthongs and diphthongs in /h_d/ context) included dynamic formant pattern, extended vowel space area for each speaker, the amount of frequency change for F1 and F2 at two temporal points located close to vowel onset and offset (vector length), and vowel duration. These measures will establish both systemic and vowel inherent characteristics within these two varieties of English. Dialectal differences will be discussed. [Work supported by NIH NIDCD R01 DC006871.]

4pSC12. Vocal tract normalization for vowel recognition. Robert A. Houde (Ctr. for Commun. Res., 125 Tech Park Dr., Rochester, NY 14623, rahoude@gmail.com) and James M. Hillenbrand (Western Michigan Univ., Kalamazoo, MI 49008)

The vocal tract normalization problem arises from the fact that the spectrum envelopes of like vowels spoken by talkers with different vocal tract lengths appear as the same spectral pattern shifted along a log frequency scale. A narrow-band model of vowel perception was developed in which vowels are identified by comparing input narrow-band vowel spectra with broad-band pitch-independent vowel templates [J. M. Hillenbrand and R. A. Houde, J. Acoust. Soc. Am. 113, 1044-1055 (2003)]. An earlier evaluation of this model was carried out on a large database of vowels spoken by men, women, and children. In this evaluation the vocal tract normalization problem was bypassed by constructing separate templates for men, women, and children. The present study extended this work by examining two approaches to the vocal tract normalization problem: (1) An explicit normalization approach in which the spectral pattern of each input token was normalized by a factor based on the pitch of the token and (2) an approach in which templates were assembled by summing together spectral patterns that were sufficiently similar, thus creating multiple tem- plates for each vowel. Performance of the model with these two approaches to vocal tract normalization was compared to listening tests on a large database of vowels spoken by men, women, and children.

4pSC13. Salience of dynamic virtual formants in diphthongs. Robert Allen Fox, Ewa Jacewicz, and Chiung-Yun Chang (Speech Percept. and Acoust. Labs., Speech and Hearing Sci., Ohio State, 1070 Carmack Rd., Columbus, OH 43210-1002, fox.2@osu.edu)

Variations in the quality of a synthesized vowel can be produced by changing the frequency of its steady-state formants or the amplitude of closely-spaced static spectral components-the latter effect a function of auditory spectral integration. Recent studies have demonstrated that dynamic modification of the amplitude ratios of these spectral components can give rise to the perception of virtual diphthongs. This study examines the extent to which spectral integration of these dynamic components is uniform across a range of frequency separations of these spectral components (previous work [Fox et al., "Title," J. Acoust. Soc. Am. 120, 3252 (2006)] demonstrated the integration of static components beyond 3.5 bark). Two synthetic vowel series were created by modifying the rise or fall of F2 producing [əi]-[ə:]-[əu] and [iə]-[ə:]-[uə] series. Next, several different series were created in which this dynamic F2 was replaced by two static spectral components whose amplitude ratios were varied over time (virtual F2 glides). The spectral separation of these two components varied from 2.0 to 4.0 bark. A single-interval 2AFC identification task was used to obtain estimates of vowel quality. Results will be discussed in terms of whether the spectral integration effects decline as the frequency separation between components increases. [Work supported by NIDCD R01DC00679-01A1.]

4pSC14. Formant amplitude in the perception of $/ \mathrm{i} /$ and $/ \mathrm{u} /$. Michael Kiefte, Teresa Enright, and Lacey Marshall (School of Human Commun. Disord., Dalhousie Univ., Halifax, NS B3H 1R2, Canada)

Although recent evidence reconfirmed the importance of spectral peaks in vowel identification [M. Kiefte and K.R. Kluender, J. Acoust. Soc. Am. 117, 1395-1404 (2005)], the roles of formant bandwidth and amplitude in perception have not been firmly established. Although several studies have shown changes in vowel categorization with changes in bandwidth and amplitude, such manipulations often result in peaks that have very little local spectral contrast or which may be perceptually masked by other spectral prominences. This study compares local contrast and masking of spectral peaks in vowel perception. A stimulus continuum ranging from /i/ to /u/ in both the amplitude of the second formant as well as that of higher formants was presented to listeners for identification. Both fullspectrum and incomplete-spectrum stimuli were used to demonstrate the effects of local spectral contrast and simultaneous masking. A second experiment was used to determine if listeners were able to detect the presence of the second spectral peak in the same stimuli. Results from both experiments indicated that both masking and spectral contrast play a role in vowel perception.

4pSC15. Effects of dialect variation on speeded word classification. Cynthia G. Clopper (Dept. of Linguist., Ohio State Univ., 1712 Neil Ave., Columbus, OH 43210, clopper.1@osu.edu)

Dialect variation has been found to significantly affect speech intelligibility in noise. The current experiment was designed to examine the effects of dialect variation on the processing of a single phonemic contrast. Participants were presented with tokens of the words "bad" and "bed" produced by talkers from Chicago and Indianapolis. The vowels in the Chicago tokens were shifted according to the Northern Cities shift (raised and fronted "bad," lowered and backed "bed"). The Indianapolis tokens were unshifted. The participants were asked to classify each token as "bad" or "bed" as quickly as possible in three conditions: Chicago tokens only, Indianapolis tokens only, or mixed Chicago and Indianapolis tokens. 
While overall accuracy was high in all three conditions, participants were slower in the Chicago-only and mixed conditions than in the Indianapolisonly condition. In addition, participants responded more slowly to the Indianapolis tokens in the mixed condition than in the Indianapolis-only condition, but response times to the Chicago tokens did not differ across the two conditions. These results suggest that acoustic-phonetic dialect differences interfere with lexical processing and that global effects of dialect variation on speech intelligibility may reflect confusions between specific pairs of phonemes as a result of subphonemic vowel shifts.

4pSC16. Cross-linguistics differences in non-speech perception. Molly Babel and Keith Johnson (Univ. of California, Berkeley, Berkeley, CA94709-2650, mbabel@berkeley.edu)

This research explores listeners perception of non-speech stimuli across languages. We will report on two experiments conducted with the same stimuli. The stimuli were synthesized from naturally produced vowel-fricative-vowel sequences consisting the fricatives [f, th, s, sh, $\mathrm{x}, \mathrm{h}]$, and the vowel environments [i_i, a_a, u_u]. The vowels were synthesized using sine wave synthesis and appended onto the natural fricative. Tokens will be presented to two groups of Dutch and English listeners over headphones with a $100 \mathrm{~ms}$ interval. Listeners will be told that the stimuli is non-speech. The first experiment is a simple AX discrimination task where the listener decides whether the tokens were same or different. The second experiment is a rating task where the listener is asked to rate the similarity of the two tokens on a five-point equal interval scale. It is predicted that results of both experiments will be the same for both groups of listeners. These results will be compared to the results of the same experiment with non-synthesized speech [K. Johnson and M. Babel, J. Acoust. Soc. Am. 120, 3292 (2006)]. [Work supported by the NIH.]

THURSDAY AFTERNOON, 7 JUNE 2007

GRAND BALLROOM C, 1:30 TO 4:35 P.M.

\title{
Session 4pSP
}

\section{Signal Processing in Acoustics, Acoustical Oceanography, and Underwater Acoustics: Signal Processing and Uncertainty II}

\author{
Lee Culver, Cochair \\ Pennsylvania State Univ., Applied Research Lab., P. O. Box 30, State College, PA 16804-0030 \\ Ning Xiang, Cochair \\ Rensselaer Polytechnic Inst., Architecture, 110 8th St., Greene Bldg., Troy, NY 12180 \\ Invited Papers
}

$1: 30$

4pSP1. Some speculations on source localization in uncertain ocean environments. Steven Finette (Acoust. Div. Naval Res. Lab, Washington, DC 20375)

Incomplete environmental knowledge is a factor that can adversely affect the prediction capability of physics-based signal processors to localize acoustic sources in ocean waveguides. Environmental uncertainty effectively introduces unwanted additional degrees of freedom into the signal processing scheme. Including a quantitative measure of this incomplete information into the processor can be accomplished by considering the influence of alternative environments, each weighted by their probability of occurrence. An approach is outlined to account for environmental uncertainty in matched field processing by the use of polynomial chaos basis expansions to represent the replica vectors in terms of stochastic Green's functions. These bases are interpreted as representing the effect of introducing additional degrees of freedom due to environmental uncertainty, because the pressure field replicas are specified as random processes. In this manner, a processor correlates the measured pressure field with a set of statistically weighted propagators between a replica source location and a sensor array. [Work sponsored by ONR through NRL base funding.]

\section{1:50}

4pSP2. Wigner approximation for filtered random functions and wave propagation. Patrick J. Loughlin (Dept. of Elec. and Computer Eng., Univ. of Pittsburgh, 348 Benedum Hall, Pittsburgh, PA 15261, loughlin@engr.pitt.edu)

We consider the time-varying (Wigner) spectrum of a filtered signal and derive an approximation that shows the effects of the magnitude and phase of the filter on the time-varying spectrum of the original signal. Both the deterministic and random cases are considered. Application to wave propagation with dispersion and damping is discussed. 
4pSP3. Ensemble-based Bayesian detection and tracking. Brian La Cour (Appl. Res. Labs., Univ. of Texas, P.O. Box 8029, Austin, TX 78713-8029)

This paper introduces a novel approach to Bayesian tracking in which the posterior distribution is represented by a random sample or ensemble of possible target states. Such a representation may be viewed as a special case of the traditional particle filter approach wherein all particles maintain strictly uniform weights. Measurement updates are performed using a Markov chain Monte Carlo technique, which has been adapted to use multiple chains and a variable pseudo temperature akin to that used in simulated annealing. The general formalism is illustrated in an example of sonar-based target tracking for antisubmarine warfare. For this example, specific motion models and likelihood functions are developed for both target and clutter hypotheses. The technique is examined in the context of results from a recent multistatic seatrial using an echo repeater target and compared against those of a traditional particle filter. [This work was supported by ONR Contract No. N00014-00-G-0450-21.]

\section{2:30}

4pSP4. Use of polynomial chaos expansions in the design of uncertainty-robust matched field processing algorithms. Kevin D. LePage (Naval Res. Lab., Code 7144, 4555 Overlook Ave. SW, Washington, DC 20375)

The polynomial chaos (PC) expansion method has been recently applied to estimating the statistical properties of underwater acoustic propagation in the presence of environmental uncertainty. Here we use PC estimates of the field covariance structure to design uncertainty robust match field processing (MFP) weights using Krolik's minimum variance beamformer with sound-speed perturbation constraints (MV-SPC) [J. Acoust. Soc. Am. 92 (3), 1408-1419 (1992)]. The idea behind the MV-SPC beamformer is to realize much of the high sidelobe rejection of MV processors in environments with environmental variability by opening up the signal model to include uncertainty effects. Here we compare the performance of MV-SPC designed with an adiabatic signal uncertainty model to the same beamformer designed with the PC signal model for realizations of the signal vector obtained with fully coupled propagation models, with results showing the superiority of the PC approach. [Work supported by ONR.]

\section{Contributed Papers}

2:50

4pSP5. A ray-based blind channel estimation method in ocean sound channels. Application to high-frequency underwater telecommunications. Karim G. Sabra (Marine Physical Lab., Scripps Inst. of Oceanogr., UC San Diego, San Diego, CA 92093-0238, ksabra@mpl.ucsd.edu), David R. Dowling (Univ. of Michigan, Ann Arbor, MI), H. C. Song, W. A. Kuperman, W. S. Hodgkiss, T. Akal (UC San Diego, San Diego, CA 92093-0238), J. M. Stevenson, and Piero Guerrini (NATO Undersea Res. Ctr., La Spezia, Italy)

A signal that travels through a sound channel is typically distorted when recorded by a remote listener because of interference arising from multiple propagation paths. We developed a ray-based blind channel estimation technique in ocean sound channels, i.e., using only records of the distorted signal along a transducer array. The technique yields broadband estimates of the source-to-array impulse response (or Green's functions) synthesized directly from the recorded distorted signals. The technique relies on separating out the contribution of a propagating ray path having a phase that depends linearly on frequency. This technique was used to decode high-frequency underwater telecommunications sequences collected in 2005 off the West Coast of Italy, without any a priori knowledge of the channel impulse response. [Work supported by ONR.]

$$
\text { 3:05-3:20 Break }
$$

\section{$3: 20$}

4pSP6. Probabilistic descriptions of low-frequency passive sonar signals. Colin W. Jemmott, Jeffrey A. Ballard, R. Lee Culver, and Leon H. Sibul (Appl. Res. Lab. and Grad. Program in Acoust., The Penn State Univ., P.O. Box 30, State College, PA 16804)

The overall goal of our research is to develop a method whereby knowledge of environmental variability is incorporated into a passive sonar detector. The Estimated Ocean Detector (EOD) is a maximum likelihood receiver that incorporates statistical knowledge of signal uncertainty in the form of signal parameter probability density functions (pdf's). The structure and performance of the EOD depends fundamentally on accurate probabilistic models of received signal and noise parameters, the so-called prior distributions. Previous work has concentrated on Monte Carlo to make use of deterministic and statistical knowledge of the uncertain and variable ocean environment to predict signal parameter pdf's. This talk presents a statistical analysis of signals recorded during the SWellEX-96 measurement that took place in shallow water off the coast of California. The data consist of $\mathrm{CW}$ signals transmitted simultaneously from moving sources at two different depths and received at a horizontal array. The statistical analysis includes estimates of time-varying moments of signal parameters and coherence of signal amplitude, frequency, and phase. [Work supported by ONR Undersea Signal Processing Code 321US.]

\section{3:35}

4pSP7. Environmental and signal models for acoustic models for acoustic propagation through a variable ocean. Richard Lee Culver and H. John Camin (Appl. Res. Lab. and Grad. Program in Acoust., Penn State Univ., P.O. Box 30, State College, PA 16804, rlc5@psu.edu)

Acoustic signals propagating through the ocean are refracted, scattered, and attenuated by the ocean volume and boundaries. Although much about the ocean environment and its effect on acoustic propagation is understood, there is always variability associated with both environment parameters and the received signal, and uncertainty in our knowledge of both. Environment and signal models (e.g., sound speed, bottom loss, and received level) often include only mean values, but they can include the higher order moments that parametrize variability. Interest in relating environmental and signal models and variability is motivated by recent efforts to capture ocean environmental uncertainty that have enhanced understanding and models of environmental parameters, and by advances in ocean acoustic signal processing that indicate how to exploit knowledge of signal variability in order to improve sonar performance. These two developments can be connected if the relationship between environmental and signal parameters (or models) is understood. We show that when a signal prediction must reflect realistic, environment-driven variability over a particular space-time interval, the environmental models used to construct the signal model must contain the environmental variability present during this same interval. [Work supported by ONR Undersea Signal Processing Code 321US.] 
4pSP8. Classification of active sonar detections with class specific method. Charles F. Gaumond and Ralph N. Baer (Acoust. Div. Naval Res. Lab., 4555 Overlook Ave. SW, Washington, DC 20375-5320)

The use of the Class Specific Method (CSM) [P. M. Baggenstoss, Trans. Sig. Proc. 47, 3428-3432 (1999)] is demonstrated with numerically simulated data. The two models of a cylindrical shell and a fish school are shown to generate sufficient statistics with appropriate assumptions. The common reference signal for the CSM is Gaussian white noise. An interpretation of the resulting conditional probability density functions is given, which leads to a numerical regularization. The resulting implementation of the CSM is demonstrated using numerically modeled data of variable bandwidth with variable amounts of additive Gaussian white noise. This approach enables the evaluation of bandwidth requirements for sufficient classification performance. The method is shown to be generalizable to include effects of propagation. [Work supported by the Office of Naval Research.]

\section{4:05}

4pSP9. Statistical characterization of very low frequency communication channels at ocean basin-scales. John Spiesberger (Dept. Earth and Env. Sci., Univ. of Pennsylvania, Philadelphia, PA 19104-6316, johnsr@sas.upenn.edu) and Dale Green (Teledyne Benthos Inc., North Falmouth, MA 02556)

The bit rate for incoherent communication is estimated for low frequency sounds $(75 \mathrm{~Hz}, 0.03 \mathrm{~s}$ resolution) transmitted $3115 \mathrm{~km}$ between a bottom-mounted source and a towed array. A model for short term fluctuations of sound agrees with measurements without tuning the model to the data. In particular, measured and modeled probability distributions for multipath coherence time and amplitude fluctuations are similar. Temporal evolution of the oceanographic component of the model is governed by the linear dispersion relation for internal gravity waves. The acoustic component of the model is based on the sound-speed insensitive parabolic approximation. This is the third attempt to use the model to predict shortterm acoustic fluctuations at basin-scales. All three attempts yielded results similar to measurements. If further comparisons between the model and data are successful, the model could be used to design and implement low frequency communication systems. A simulation yields 4 bits per second for a Benthos modem using an incoherent communication scheme via frequency shift keying. [Work supported by the ONR.]

\section{$4: 20$}

4pSP10. Some effects of spatial randomness along the length of the cochlear on its performance. Stephen Elliott, Emery $\mathrm{Ku}$, and Ben Lineton (ISVR, Univ. of Southampton, Southampton, UK)

If the active cochlear is modelled as having a uniform distribution of parameters, the system is stable even for high gains in the cochlear amplifiers, resulting in very significant active enhancements of its response, of the order of $90 \mathrm{~dB}$. In a real biological system, however, such uniform distributions will always be disrupted by random spatial variations, and a state space model of the cochlea has been developed that can be used to investigate the effects of such random variations. One effect is found to be that the cochlear amplifier gains have to be reduced to maintain stability, so that maximum active enhancements of the cochlear response is more typically $40 \mathrm{~dB}$. The cochlear amplifier, however, is nonlinear as well as active, and unstable poles in the linear model generate instabilities that settle into limit cycle oscillations at discrete frequencies in the nonlinear model. These oscillations are thought to be the origin of spontaneous otoacoustic emissions, and have a significant effect on sound detection at low level. At higher sound pressure levels the nonlinearity in the cochlear amplifier suppresses such oscillations and the sound detection has a more uniform frequency response, although the amplification is reduced. 